\title{
A stereoselective aza-Prins reaction: rapid access to enantiopure pi- peridines and pipecolic acids
}

\author{
Ramana Reddy Mittapalli ${ }^{\mathrm{a}}$, Simon J. Coles ${ }^{\mathrm{b}}$, Wim T. Klooster ${ }^{\mathrm{b}}$ and Adrian P. Dobbs ${ }^{\mathrm{a} *}$

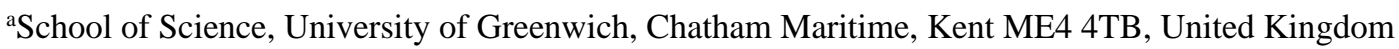 \\ ${ }^{b}$ UK National Crystallography Service, University of Southampton, University Road, Southampton, SO17 1BJ, United Kingdom
}

KEYWORDS aza-Prins reaction, asymmetric synthesis, piperidines, pipecolic acids

\begin{abstract}
The aza-Prins reaction is a widely employed and highly efficient method for the preparation of saturated nitrogencontaining heterocycles. Its major drawback has always been a lack of diastereoselectivity and the formation of racemic products. Herein we address these problems and report, for the first time, the synthesis of both diastereomerically and enantiopure multiplysubstituted piperidines via the aza-Prins reaction. This method will be widely applicable for natural product synthesis and is exemplified here by the synthesis of enantiopure pipecolic acid derivatives.
\end{abstract}

\section{INTRODUCTION}

Nitrogen heterocycles are amongst the most important structural component in both natural products and modern pharmaceuticals. ${ }^{1}$ For example, 2,6-disubstituted and 2,4,6-trisubstituted piperidines are found widely in alkaloids. Analysis of all F.D.A. approved drugs show that $59 \%$ of small molecule drugs contain a nitrogen heterocycle and that 72 drugs contain a piperidine moiety, making it the most prevalent nitrogen heterocycle in approved drugs (Figure 1a). ${ }^{1}$ The two most common substitution patterns are $N$ - and $\mathrm{C}(4)$-, followed by $\mathrm{C}(2)$ and $\mathrm{C}(3)$ (Figure 1b). The vast majority of these drugs require careful stereocontrol of the substitutents around the ring during manufacture. Methods for their synthesis have been widely reviewed ${ }^{2-5}$ but the greatest problem has always been the asymmetric construction of the piperidine ring. Furthermore, there is an emerging need for $\mathrm{sp}^{3}$-rich heterocyclic frameworks, again with both relative and absolute stereocontrol of the substituents. a)<smiles>C1CCNCC1</smiles>

$$
\text { piperidine }
$$

72

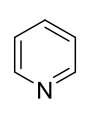

62 pyridine

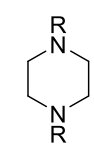

piperazine
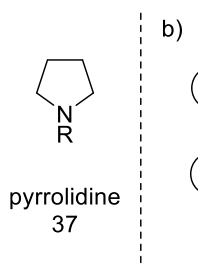

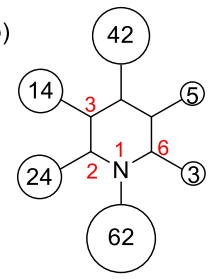

FIGURE 1: a) most abundant nitrogen heterocycles within FDA approved drugs and their occurrence; b) most popular positions of piperidine substitution. ${ }^{1}$

Since it was first reported by Hanschke in 1955, the Prins cyclisation has become one of the leading methods for the preparation of tetrahydropyran rings. ${ }^{6}$ For many years this remained a highly diastereoselective but racemic reaction. A limited number of asymmetric cyclisation processes to give enantiopure tetrahydropyrans have been reported, either commencing from enantiopure starting materials ${ }^{7-9}$ or employing an asymmetric catalyst approach. ${ }^{10-13}$ More recently, the azaPrins $^{14-21}$ (and related aza-silyl-Prins ${ }^{22-25}$ ) cyclisation - the reaction of a homoallylic amine and aldehyde (or acetal or epoxide) promoted by a Lewis or Brønsted acid (Scheme 1) - has emerged as a highly efficient method for the diastereoselective synthesis of substituted piperidines, albeit in racemic form. While methods for the asymmetric synthesis of heterocycles via chiral iminium ion cyclisations have been extensively reviewed $^{26}$, there are only isolated examples of attempts at simple asymmetric aza-Prins reactions and there remains no general catalytic and asymmetric aza-Prins cyclisation reaction and interest in developing enantioselective aza-Prins processes is high and very challenging. Recently we have reported a related asymmetric aza-silyl-Prins reaction ${ }^{27}$ and Maruoka has described an aza-Prins endo-type cyclisation of 2-(1-phenylvinyl)benzaldehyde and $\mathrm{BocNH}_{2}$ catalysed by a BINOL-derived $N$-triflyl phosphoramide to form 1-aminoindenes. ${ }^{28}$

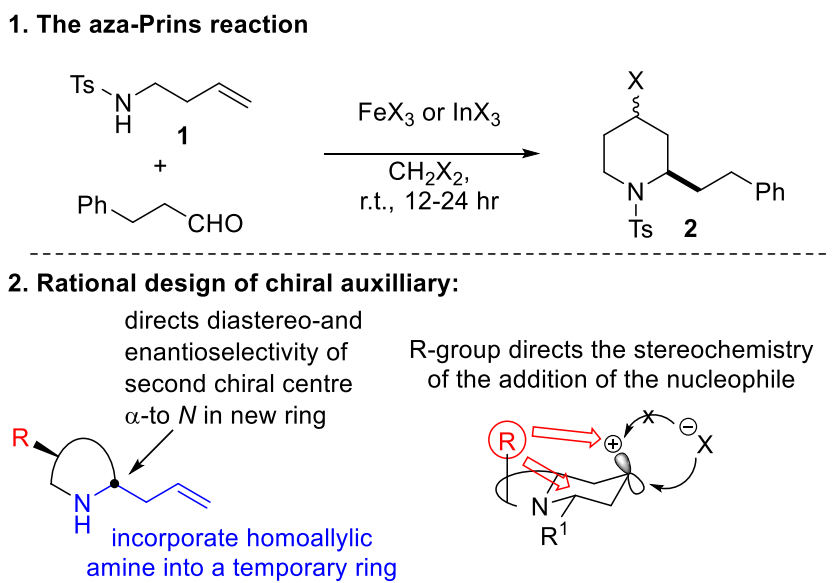


SCHEME 1: 1 . The aza-Prins reactions, giving a diastereomeric mixture through non-selective trapping at c-4. 2. Rationale in designing a chiral auxiliary to direct C-4 trapping.

Herein we report for the first time a highly novel, efficient and high yielding diastereo- and enantioselective aza-Prins reaction, offering the ability to access enantiopure multi-substituted $\mathrm{sp}^{3}$-rich piperidines.

\section{RESULTS AND DISCUSSION}

In designing an asymmetric aza-Prins reaction, we were conscious that one of the considerable drawbacks of the aza-Prins cyclisation has been the necessity for an $N$-sulfonamide ${ }^{14-15}$ group (Ts, Ns, Bs) in the homoallylic amine component (1); groups such as $N$-benzyl or $N$-Boc do not undergo aza-Prins cyclisation. ${ }^{17,20,29}$ Further, we have recently reported that chiral auxiliaries on nitrogen, for example $\alpha$-methylbenzyl, in place of the tosyl group, were inefficient for promoting asymmetric aza-silyl-Prins-type reactions. ${ }^{27}$ Therefore we have instead focused on developing a novel, easy to prepare and use chiral-auxilliary-containing a homoallylic amine. We have previously shown that the aza-Prins and aza-silyl-Prins reactions are 2,6-trans selective across the nitrogen ${ }^{17,23,27,30-31}$ and reasoned that this trans relationship would be maintained when employing a chiral centre adjacent to nitrogen in the homoallylic amine starting material and thus generating a new chiral centre across the nitrogen after cyclisation.

In order to test this hypothesis, starting from $(R)$-allyl glycine, it was possible to prepare $(R)$-allylmorpholin-2-one $(3)$ in a single step. Attempting the aza-Prins reaction using $\mathrm{FeCl} 3$ and phenyl propionaldehyde afforded the corresponding piperidine (4, Scheme 2$)$ in $63 \%$ yield, with the expected exclusive trans-relationship of substituents across the nitrogen, but surprisingly with little selectivity at the C-4 position compared with employing (1). We reasoned that the introduction of either one or two substituents on the two bridging carbons in (3) may improve the $\mathrm{C}-4$ selectivity of the aza-Prins reaction.

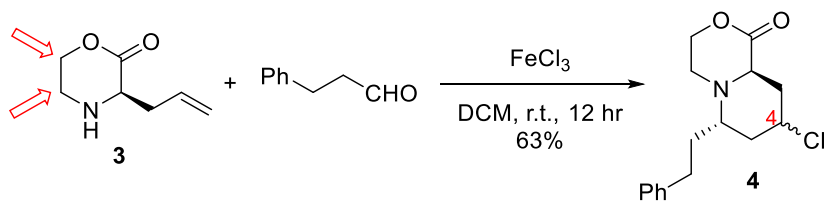

SCHEME 2: 2,6-trans selective aza-Prins reaction starting from $(R)$-allylmorpholin-2-one generates an equal mixture of adducts at $\mathrm{C}-4$.

Therefore we prepared mono- $(\mathbf{8})^{32}$ and di-phenyl (7) ${ }^{33-34}$ allylmorpholin-2-ones in both enantiomeric forms in just 4 steps, as two new chiral platforms containing a homoallylic amine. Following the rationale presented in Scheme 1(2), the phenyl substituents were chosen as these would both facilitate rapid removal of part of the chiral platform post Prins cyclisation and crucially block one face from anion trapping. Treatment of either (7) or (8) with NaHMDS and an allyl or propargylic bromide gave a small library of enantiomerically pure secondary amines (Table 1).

Table 1: Synthesis of chiral homoallylic amines

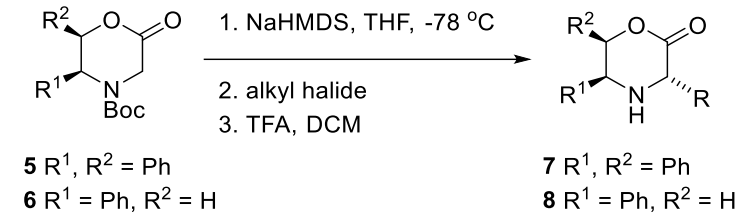

\begin{tabular}{|l|l|l|l|l|l|l|}
\hline $\begin{array}{l}\mathbf{E} \\
\text { nt } \\
\text { ry }\end{array}$ & $\mathbf{R}^{\mathbf{1}}$ & $\mathbf{R}^{\mathbf{2}}$ & $\begin{array}{l}\text { Alkyl } \\
\text { halide }\end{array}$ & $\begin{array}{l}\text { Pro } \\
\text { duc } \\
\mathbf{t}\end{array}$ & $\begin{array}{l}\text { \% } \\
\text { Yie } \\
\mathbf{l d}^{\mathbf{a}}\end{array}$ \\
\hline 1. & $\mathrm{Ph}$ & $\mathrm{Ph}$ & $\begin{array}{l}\text { Allyl } \\
\text { bromide }\end{array}$ & $\mathrm{CH}_{2} \mathrm{CHCH}_{2}$ & 7 & 81 \\
\hline 2. & $\mathrm{Ph}$ & $\mathrm{H}$ & $\begin{array}{l}\text { Allyl } \\
\text { bromide }\end{array}$ & $\mathrm{CH}_{2} \mathrm{CHCH}_{2}$ & $8 \mathrm{a}$ & 85 \\
\hline 3. & $\mathrm{Ph}$ & $\mathrm{H}$ & $\begin{array}{l}\text { Propar- } \\
\text { gyl bro- } \\
\text { mide }\end{array}$ & $\mathrm{CH}_{2} \mathrm{CCH}$ & $8 \mathrm{~b}$ & 85 \\
\hline 4. & $\mathrm{Ph}$ & $\mathrm{H}$ & $\begin{array}{l}\text { Crotyl } \\
\text { bromide }\end{array}$ & $\begin{array}{l}\mathrm{CH}_{2} \mathrm{CHCH}(\mathrm{C} \\
\left.\mathrm{H}_{3}\right)\end{array}$ & $8 \mathrm{c}$ & 86 \\
\hline 5. & $\mathrm{Ph}$ & $\mathrm{H}$ & $\begin{array}{l}3- \\
\text { Bromo- } \\
2- \\
\text { methylpr } \\
\text { op-1-ene }\end{array}$ & $\begin{array}{l}\mathrm{CH}_{2} \mathrm{C}\left(\mathrm{CH}_{3}\right) \\
\mathrm{CH}_{2}\end{array}$ & $8 \mathrm{~d}$ & 90 \\
\hline 6. & $\mathrm{Ph}$ & $\mathrm{H}$ & $\begin{array}{l}1- \\
\text { Bromo- } \\
3- \\
\text { methyl- } \\
\text { but-2- } \\
\text { ene }\end{array}$ & $\begin{array}{l}\mathrm{CH}_{2} \mathrm{CHC}_{2} \mathrm{CH}_{3} \\
)_{2}\end{array}$ & $8 \mathrm{e}$ & 80 \\
\hline
\end{tabular}

a) isolated and purified yields. b) all products isolated as a single enantiomer and diastereoisomer.

We attempted aza-Prins reactions utilizing (7a) with a range of aldehydes using Brønsted and Lewis acids to promote the reaction. With TFA, the reaction of (7a) with butanal proceeded smoothly at room temperature to afford $76 \%$ of an enantiomerically pure single diastereoisomer $(\mathbf{9 a})$, with only traces of the opposite C-4 diastereomer being isolated and which could easily be separated by chromatography (Table 2 Entry 1 ). Starting from the opposite enantiomer (7b) gave the enantiomeric product (Table 2 Entry 2). The same result was observed with a number of other aldehydes, all proceeding in excellent yields and diastereoslectivities and giving the product as a single enantiomer in every case (Table 2, Entries 3-8). The structure of $(\mathbf{9 g})$ was confirmed by x-ray crystallography and also studied by 2D NMR and NOESY experiments. This data was used to confirm the structural assignments for all compounds (9a-g) as they gave almost identical 2D and NOESY spectra to the spectra obtained for $(\mathbf{9 g})$ where xray crystallography also confirmed the structure. The use of glyoxylic acid gave a tricyclic product, whereby the acid moiety had trapped the intermediate carbocation intramolecularly (Table 2 Entry 9). Replacing TFA with pTSA gave the -OTs trapped adduct but as an inseparable mixture of diastereoisomers (Table 2 Entry 10). Finally, acetals could be employed in place of aldehydes and also gave high yields of a single enantiomerically pure product (Table 2 Entry 11).

Table 2: Asymmetric aza-Prins reactions employing Brønsted acids 


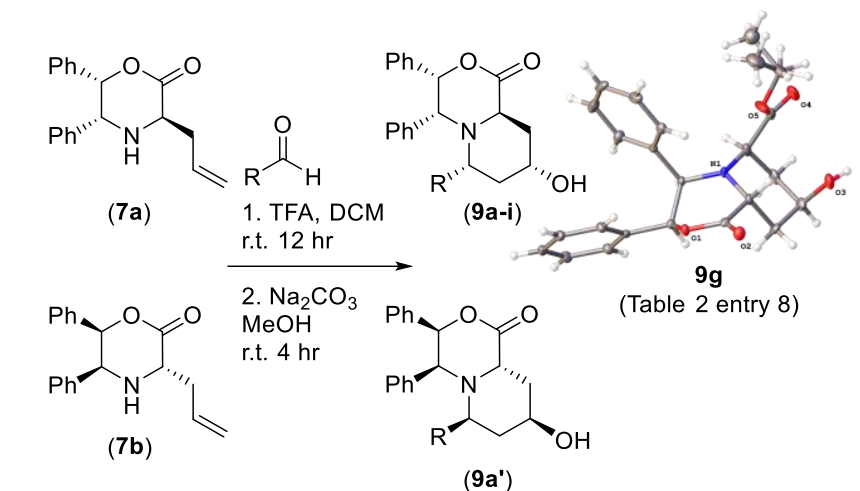

(9a')

\begin{tabular}{|c|c|c|c|c|}
\hline $\begin{array}{l}\text { En- } \\
\text { try }\end{array}$ & $\begin{array}{l}\text { Start- } \\
\text { ing } \\
\text { Mate- } \\
\text { rial }\end{array}$ & Aldehyde $^{a}$ & $\begin{array}{l}\text { Product } \\
\mathrm{R}=\end{array}$ & $\begin{array}{l}\% \\
\text { Yield }^{\mathrm{a}, \mathrm{b}}\end{array}$ \\
\hline 1. & $7 a$ & Butanal & $\mathrm{CH}_{2} \mathrm{CH}_{2} \mathrm{CH}_{3}(\mathbf{9 a})$ & 76 \\
\hline 2. & $7 b$ & Butanal & $\mathrm{CH}_{2} \mathrm{CH}_{2} \mathrm{CH}_{3}\left(\mathbf{9} \mathbf{a}^{\prime}\right)$ & 82 \\
\hline 3. & $7 a$ & $\begin{array}{l}\text { 3-Phe- } \\
\text { nylpropio- } \\
\text { naldehyde }\end{array}$ & $\mathrm{CH}_{2} \mathrm{CH}_{2} \mathrm{Ph}(\mathbf{9 b})$ & 81 \\
\hline 4. & $7 a$ & Dodecanal & $\begin{array}{l}\mathrm{CH}_{2}\left(\mathrm{CH}_{2}\right)_{9} \mathrm{CH}_{3} \\
(\mathbf{9 c})\end{array}$ & 75 \\
\hline 5. & $7 a$ & Decanal & $\begin{array}{l}\mathrm{CH}_{2}\left(\mathrm{CH}_{2}\right)_{7} \mathrm{CH}_{3} \\
(\mathbf{9 d})\end{array}$ & 73 \\
\hline 6. & $7 a$ & Octanal & $\begin{array}{l}\mathrm{CH}_{2}\left(\mathrm{CH}_{2}\right)_{5} \mathrm{CH}_{3} \\
(\mathbf{9 e})\end{array}$ & 72 \\
\hline 7. & $7 a$ & $\begin{array}{l}\text { Diphe- } \\
\text { nylacetal- } \\
\text { dehyde }\end{array}$ & $\mathrm{CHPh}_{2}(\mathbf{9 f})$ & 78 \\
\hline 8. & $7 a$ & $\begin{array}{l}\text { Ethyl gly- } \\
\text { oxalate }\end{array}$ & $\mathrm{CO}_{2} \mathrm{Et}(\mathbf{9 g})$ & $91^{\mathrm{c}}$ \\
\hline 9. & $7 a$ & $\begin{array}{l}\text { Glyoxylic } \\
\text { acid }\end{array}$ & (9h) & 60 \\
\hline 10. & $7 a$ & Octanal & $\begin{array}{l}\left.\mathrm{Ph}_{3} \cdot \mathrm{CH}_{2}\right)_{6}^{\prime \prime \prime} \\
(\mathbf{9 i})\end{array}$ & $\begin{array}{l}68^{d} \\
(65: 35)\end{array}$ \\
\hline 11. & $7 a$ & $\begin{array}{l}\text { Butanal } \\
\text { diethyl ac- } \\
\text { etal }\end{array}$ & $\mathrm{CH}_{2} \mathrm{CH}_{2} \mathrm{CH}_{3}(\mathbf{9 a})$ & 73 \\
\hline
\end{tabular}

a) isolated and purified yields. b) all products isolated as a single enantiomer and diastereoisomer. c) structure confirmed by x-ray crystallography. ${ }^{35} \mathrm{~d}$ ) using TsOH instead of TFA, giving 65:35 inseperable mixture

In the absence of a suitable nucleophile, it is also known that solvents, such as acetonitrile or benzene, can trap the cyclic secondary carbocation, in an aza-Prins-Ritter or aza-PrinsFriedel-Crafts cascade. ${ }^{21,36}$ Using pTSA in acetonitrile gave good yields of the 4-NHAc trapped products (Table 3), again as a single enantiomer and diastereomer.
Table 3: Asymmetric aza-Prins-Ritter Reactions<smiles>C=CCC1N[C@@H](c2ccccc2)[C@H](c2ccccc2)OC1=O</smiles>
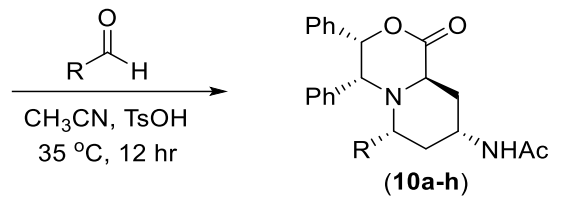

\begin{tabular}{|l|l|l|l|l|}
\hline $\begin{array}{l}\text { En- } \\
\text { try }\end{array}$ & $\begin{array}{l}\text { Star } \\
\text { ting } \\
\text { Ma- } \\
\text { te- } \\
\text { rial }\end{array}$ & Aldehyde & $\begin{array}{l}\text { Pro } \\
\text { duc } \\
\text { t }\end{array}$ & $\begin{array}{l}\text { Yield } \\
\text { a,b }\end{array}$ \\
\hline 1. & $7 \mathrm{a}$ & 3-Phenylpropionaldehyde & $\mathbf{1 0 a}$ & 83 \\
\hline 2. & $7 \mathrm{~b}$ & 3-Phenylpropionaldehyde & $\mathbf{1 0 a}$ & 76 \\
\hline 3. & $7 \mathrm{a}$ & Octanal & $\mathbf{1 0 b}$ & 73 \\
\hline 4. & $7 \mathrm{a}$ & Decanal & $\mathbf{1 0 c}$ & 78 \\
\hline 5. & $7 \mathrm{a}$ & Hexanal & $\mathbf{1 0 d}$ & 75 \\
\hline 6. & $7 \mathrm{a}$ & Butanal & $\mathbf{1 0 e}$ & 74 \\
\hline 7. & $7 \mathrm{a}$ & Butanal diethyl acetal & $\mathbf{1 0 e}$ & 65 \\
\hline 8. & $7 \mathrm{a}$ & Phenylacetaldehyde & $\mathbf{1 0 f}$ & 82 \\
\hline 9. & $7 \mathrm{a}$ & Styrene oxide & $\mathbf{1 0 f}$ & 63 \\
\hline 10. & $7 \mathrm{a}$ & Diphenylacetaldehyde & $\mathbf{1 0 g}$ & 78 \\
\hline
\end{tabular}

a) isolated and purified yields. b) all products isolated as a single enantiomer and diastereoisomer.

In place of TFA or TsOH, the alternative is to use a Lewis acid to promote the aza-Prins cyclisation, with iron and indium trihalides being particularly prevalent in the literature. In these cases, the Lewis acid provides a nucleophile to trap the secondary carbocation. When initially trialing Lewis acids, a disappointing 62:38 ratio of diastereoisomers was obtained when starting from (7b) and iron trichloride (Table 4, Entry 1). Somewhat surprisingly, changing to the mono-phenyl amine (8) gave much improved results and a single diastereomer product in excellent yield. However, the configuration of the halogen is the opposite to that observed for the diphenyl starting materials (7a/7a'). Both iron trichloride and iron tribromide gave excellent yields of 4-halo products (12a-d, 13a-b), as long as the appropriate halide-containing solvent was employed, to avoid mixed-halogen products (Table 3 ). The structure of (12d) was confirmed by x-ray crystallography and also by 2D NMR and NOESY experiments. This combined knowledge was utilized to confirm the structural assignments for compounds (11-16b) as they gave almost identical 2D and NOESY spectra around the ring hydrogens to the spectra obtained for (12d) where x-ray crystallography had also confirmed the structure. The methodology was then extended to incorporate other nucleophiles into the mono-phenyl amine (8). Reaction of $\mathbf{8}$ with butanal in the presence of TFA ( 2 equiv.) surprisingly gave the hydroxy product (14a) in $88 \%$ yield (Table 4 entry 8 ). The same outcome was observed with other aldehydes (Table 4 entry 8 ), and an acetal (Table 4 entry 10). Replacing TFA with pTSA in DCM provided tosyl-substituted derivatives in excellent yields (15a-b, Table 4, entries 11-12). Finally, conducting the reaction in acetonitrile and optimized with boron trifluoride etherate, a sequential aza-Prins cyclisation-Ritter reaction was observed, giving single amine products in high yield and selectivity (16a-b, Table 4 entries 13-14) but with the opposite stereochemistry at C4 (c.f. 10ah). 
Table 4: Asymmetric aza-Prins reactions employing Lewis acids

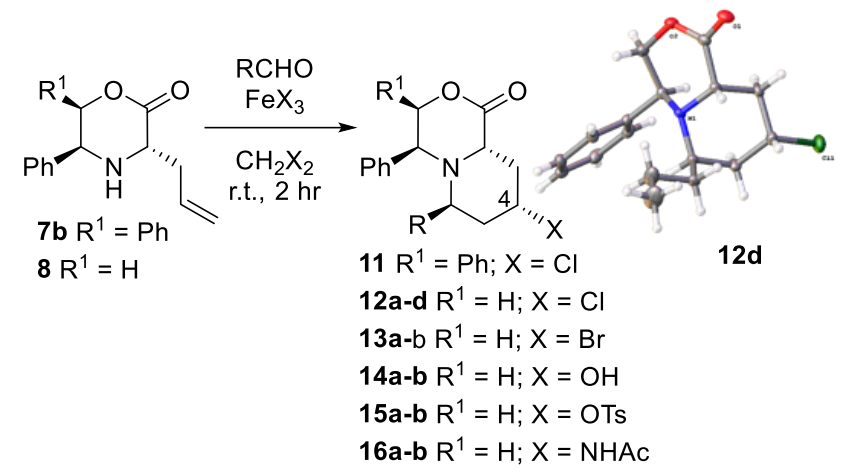

\begin{tabular}{|c|c|c|c|c|c|c|}
\hline $\begin{array}{l}\text { E } \\
\text { nt } \\
\text { ry }\end{array}$ & $\mathbf{R}^{1}$ & Aldehyde & $\mathbf{X}$ & Acid $^{\mathrm{a}}$ & $\begin{array}{l}\text { Pro } \\
\text { duc } \\
\text { t }\end{array}$ & $\begin{array}{l}\% \\
\text { Yie } \\
\text { ld }^{\text {b,c }}\end{array}$ \\
\hline 1. & $\mathrm{Ph}$ & $\begin{array}{l}\text { 3-Phe- } \\
\text { nylpropio- } \\
\text { naldehyde }\end{array}$ & $\mathrm{Cl}$ & $\mathrm{FeCl}_{3}$ & 11 & $\begin{array}{l}67 \\
\% \\
(62: \\
38)\end{array}$ \\
\hline 2. & $\mathrm{H}$ & $\begin{array}{l}\text { 3-Phe- } \\
\text { nylpropio- } \\
\text { naldehyde }\end{array}$ & $\mathrm{Cl}$ & $\mathrm{FeCl}_{3}$ & $12 a$ & 90 \\
\hline 3. & $\mathrm{H}$ & $\begin{array}{l}\text { benzalde- } \\
\text { hyde }\end{array}$ & $\mathrm{Cl}$ & $\mathrm{FeCl}_{3}$ & $12 b$ & 91 \\
\hline 4. & $\mathrm{H}$ & $\begin{array}{l}\text { Diphenyla- } \\
\text { cetaldehyde }\end{array}$ & $\mathrm{Cl}$ & $\mathrm{FeCl}_{3}$ & $12 \mathrm{c}$ & 89 \\
\hline 5. & $\mathrm{H}$ & Butanal & $\mathrm{Cl}$ & $\mathrm{FeCl}_{3}$ & 12d & $89^{\mathrm{d}}$ \\
\hline 6. & $\mathrm{H}$ & $\begin{array}{l}\text { 3-Phe- } \\
\text { nylpropio- } \\
\text { naldehyde }\end{array}$ & $\mathrm{Br}$ & $\mathrm{FeBr}_{3}$ & 13a & 90 \\
\hline 7. & $\mathrm{H}$ & $\begin{array}{l}\text { Diphenyla- } \\
\text { cetaldehyde }\end{array}$ & $\mathrm{Br}$ & $\mathrm{FeBr}_{3}$ & $13 b$ & 80 \\
\hline 8. & $\mathrm{H}$ & Butanal & $\mathrm{OH}$ & TFA & $14 a$ & 88 \\
\hline 9. & $\mathrm{H}$ & Dodecanal & $\mathrm{OH}$ & TFA & $14 b$ & 90 \\
\hline 10 & $\mathrm{H}$ & $\begin{array}{l}\text { Butanal di- } \\
\text { ethyl acetal }\end{array}$ & $\mathrm{OH}$ & TFA & $14 a$ & 76 \\
\hline 11 & $\mathrm{H}$ & Decanal & OTs & PTSA & $15 a$ & 80 \\
\hline 12 & $\mathrm{H}$ & Dodecanal & OTs & PTSA & $15 b$ & 78 \\
\hline 13 & $\mathrm{H}$ & Decanal & $\begin{array}{l}\text { NHA } \\
\mathrm{c}\end{array}$ & $\mathrm{BF}_{3} . \mathrm{OEt}_{2}$ & $16 a$ & $75^{\mathrm{e}}$ \\
\hline 14 & $\mathrm{H}$ & Dodecanal & $\begin{array}{l}\text { NHA } \\
\mathrm{c}\end{array}$ & $\mathrm{BF}_{3} . \mathrm{OEt}_{2}$ & $16 b$ & $72^{\mathrm{e}}$ \\
\hline
\end{tabular}

a) other halogen containing Lewis acids $\left(\mathrm{TiCl}_{4}, \mathrm{SnCl}_{4}, \mathrm{AlCl}_{3}\right)$ gave lower yields and decomposition of starting material; b) isolated and purified yields. c) all products isolated as a single enantiomer and diastereoisomer (except entry 1). d) structure confirmed by x-ray crystallography. ${ }^{37}$ e) $\mathrm{CH}_{3} \mathrm{CN}$ as solvent.

Substituted allyl and propargylic amines (Table 1) were the next to be employed in the cyclisation and furnished a range of substituted products (Table 5). Using (3S,5S)-3-((E)-But-2enyl)-5-phenylmorpholin-2-one ( $8 c$, Table 5 entry 1$)$ gave very high yields of the methyl-substituted product as a single diastereoisomer and enantiomer under either Lewis or Bronsted acid conditions. When using (3S,5S)-3-(3-Methylbut-2-enyl)-5-phenylmorpholin-2-one (8e, Table 5 entries 24), the formation of a 5-membered ring was observed, presumably via the more stable tertiary carbocation, and irrespective of the aldehyde or acid employed. ${ }^{17}$ (3S,5S)-3-(2-Methylallyl)-5-phenylmorpholin-2-one (8d, entries 5-8) gave a new single isomer quaternary chiral centre. Finally using $(3 S, 5 S)$ 5-Phenyl-3-(prop-2-ynyl) morpholin-2-one (8b, entry 9) gave a single vinyl halide-containing diastereoisomer and enantiomer where either $\mathrm{Cl}$ or $\mathrm{Br}$ could be incorporated into the product. The structures of (17c) and (19b) were additionally confirmed by x-ray crystallography in addition to the 2D NMR and NOESY experiments used to assign all the structures and stereochemistry in Table 5.

Table 5: Asymmetric aza-Prins reactions employing substituted alkenes or alkynes

\begin{tabular}{|c|c|c|c|c|c|}
\hline $\begin{array}{l}\text { E } \\
\text { nt } \\
\text { ry }\end{array}$ & Amine & $\begin{array}{l}\text { Alde- } \\
\text { hyde }\end{array}$ & Acid & Product & $\begin{array}{l}\% \\
\text { Yie } \\
\text { ld }^{\mathrm{a}, \mathrm{b}}\end{array}$ \\
\hline 1. & $(8 c)$ & $\begin{array}{l}\text { 3- } \\
\text { Phe- } \\
\text { nylpr } \\
\text { opio- } \\
\text { nalde- } \\
\text { hyde }\end{array}$ & $\begin{array}{l}\mathrm{FeCl}_{3} \\
\mathrm{FeBr}_{3} \\
\text { TFA }\end{array}$ & $(\mathbf{1 7 a - c )}$ & $\begin{array}{l}92 \\
(\mathrm{Cl}) \\
91 \\
(\mathrm{Br}) \\
83 \\
(\mathrm{O} \\
\mathrm{H})^{\mathrm{c}}\end{array}$ \\
\hline 2. & $(8 e)$ & $\begin{array}{l}\text { 3- } \\
\text { Phe- } \\
\text { nylpr } \\
\text { opio- } \\
\text { nalde- } \\
\text { hyde }\end{array}$ & $\begin{array}{l}\mathrm{FeCl}_{3} \\
\text { TFA }\end{array}$ & $\sum_{(\mathbf{1 8 a}, \mathbf{1 8}}$ & $\begin{array}{l}63 \\
(\mathrm{Cl}) \\
76 \\
(\mathrm{O} \\
\mathrm{H})\end{array}$ \\
\hline 3. & & $\begin{array}{l}\text { Buta- } \\
\text { nal }\end{array}$ & TFA & ${ }_{(18 c)}$ & 72 \\
\hline 4. & & $\begin{array}{l}\text { 3- } \\
\text { Phe- } \\
\text { nylpr } \\
\text { opio- } \\
\text { nalde- } \\
\text { hyde }\end{array}$ & $\begin{array}{l}\mathrm{Sc}(\mathrm{O} \\
\mathrm{Tf})_{3}\end{array}$ & (18d) & 51 \\
\hline 5. & $(\mathbf{8 d})$ & $\begin{array}{l}\text { 3- } \\
\text { Phe- } \\
\text { nylpr } \\
\text { opio- } \\
\text { nalde- } \\
\text { hyde }\end{array}$ & $\mathrm{FeCl}_{3}$ & (19a) & 76 \\
\hline 6. & & $\begin{array}{l}\text { Buta- } \\
\text { nal }\end{array}$ & & (19b) & $72^{c}$ \\
\hline
\end{tabular}




\begin{tabular}{|c|c|c|c|c|c|}
\hline 7. & & $\begin{array}{l}3- \\
\text { Phe- } \\
\text { nylpr } \\
\text { opio- } \\
\text { nalde- } \\
\text { hyde }\end{array}$ & TFA & (19c) & 80 \\
\hline 8. & & $\begin{array}{l}\text { Buta- } \\
\text { nal }\end{array}$ & & $\int_{(19 d)}^{O}$ & 75 \\
\hline 9. & (8b) & $\begin{array}{l}3- \\
\text { Phe- } \\
\text { nylpr } \\
\text { opio- } \\
\text { nalde- } \\
\text { hyde }\end{array}$ & $\begin{array}{l}\mathrm{FeCl}_{3} \\
\mathrm{FeBr}_{3}\end{array}$ & (20a, 20b) & $\begin{array}{l}70 \\
(\mathrm{Cl}) \\
71 \\
(\mathrm{Br})\end{array}$ \\
\hline
\end{tabular}

a) isolated and purified yields. b) all products isolated as a single enantiomer and diastereoisomer. c) structure confirmed by x-ray crystallography. ${ }^{38}$

The morpholin-2-one has not been considered as a chiral auxillary since part of it remains in the final product; therefore it is seen as a highly efficient chiral platform for controlling the aza-Prins reaction. Partial removal of the chiral platform was easily achieved via catalytic hydrogenation, from both the mono- and di-phenyl substituted bicyclic compounds, to afford enantiopure pipecolic acid derivatives. All substituents at the C4-position tolerated these deprotection conditions except in vinyl halo compounds 20a and 20b where the halogen was lost along with the alkene during hydrogenation (Table 6 Entry 6). Use of acidified ethanol as the solvent furnished the ethyl ester product.

Table 6: Synthesis of substituted pipecolic acids via partial removal of the chiral platform<smiles></smiles>

\begin{tabular}{|c|c|c|c|c|}
\hline Entry & $\begin{array}{c}\text { R (Starting } \\
\text { material) }\end{array}$ & $\mathbf{R}^{\mathbf{1}}$ & $\mathbf{X}^{\mathbf{a}}$ & \% Yield $^{\mathbf{b}, \mathbf{c}}$ \\
\hline 1. & $\begin{array}{c}\left(\mathrm{CH}_{2}\right)_{2} \mathrm{CH}_{3} \\
\left(\mathbf{9 a} / \mathbf{9} \mathbf{a}^{\prime}\right)\end{array}$ & $\mathrm{Ph}$ & $\mathrm{OH}$ & $\begin{array}{c}95 / 93^{\mathrm{d}} \\
(\mathbf{2 1 a})\end{array}$ \\
\hline 2. & $\begin{array}{c}\left(\mathrm{CH}_{2}\right)_{6} \mathrm{CH}_{3} \\
(\mathbf{9 e})\end{array}$ & $\mathrm{Ph}$ & $\mathrm{OH}$ & $\begin{array}{c}92 \\
(\mathbf{2 1 b})\end{array}$ \\
\hline 3. & $\begin{array}{c}\left(\mathrm{CH}_{2}\right)_{2} \mathrm{Ph} \\
(\mathbf{1 0 a} / \mathbf{1 0 a})\end{array}$ & $\mathrm{Ph}$ & $\mathrm{NHAc}$ & $\begin{array}{c}91 / 89 \\
\left(\mathbf{2 1 c} / \mathbf{2 1} \mathbf{c}^{\prime}\right)\end{array}$ \\
\hline 4. & $\begin{array}{c}\left(\mathrm{CH}_{2}\right)_{2} \mathrm{CH} \\
(\mathbf{1 4 a})\end{array}$ & $\mathrm{H}$ & $\mathrm{OH}$ & $\begin{array}{c}76 \\
(\mathbf{2 1 d})\end{array}$ \\
\hline 5. & $\begin{array}{c}\left(\mathrm{CH}_{2}\right)_{2} \mathrm{Ph} \\
(\mathbf{1 2 a})\end{array}$ & $\mathrm{H}$ & $\mathrm{Cl}$ & $\begin{array}{c}68^{\mathrm{e}} \\
(\mathbf{2 1 e})\end{array}$ \\
\hline 6. & $\begin{array}{c}\left(\mathrm{CH}_{2}\right)_{2} \mathrm{Ph} \\
(\mathbf{2 0 a} / \mathbf{2 0 b})\end{array}$ & $\mathrm{H}$ & $\mathrm{H}$ & $\begin{array}{c}51 / 57 \\
(\mathbf{2 1 f})\end{array}$ \\
\hline
\end{tabular}

a) stereochemistry of $\mathrm{X}$ in starting material different depending on if the mono-phenyl or di-phenyl compound was used (Table 2 c.f. Table
4); stereochemical integrity of $X$ from the starting material was maintained in all products. b) isolated and purified yields. c) all products isolated as a single enantiomer and diastereoisomer. d) reaction performed on both enantiomers of starting material (93\% for $(2 S, 4 S, 6 R)$ product and $95 \%$ for $(2 R, 4 R, 6 S)$-product. e) product isolated as the ethyl ester, using acidified ethanol (EtOH-HCl)

\section{CONCLUSIONS}

In summary, we have reported a highly efficient asymmetric aza-Prins reaction, capable of furnishing enantiopure piperidines and pipecolic acid derivatives in excellent yields and as a single enantiomer and diastereomer. We believe that this method will be of great use in total synthesis and the development of novel 3D structures, exploring new chemical space and we shall report our findings in due course.

\section{EXPERIMENTAL SECTION}

General methods. All dry solvents were prepared/dried according to standard procedures. Reactions were performed in oven-dried round bottom flasks. The flasks were fitted with rubber septa and the reactions were conducted under nitrogen atmosphere. Reaction mixtures were purified by flash column chromatography on silica gel 100-200 mesh. TLC plates were visualized by exposure to ultraviolet light and/or by exposure to acidic ethanolic solution of ninhydrin followed by heating ( $<1 \mathrm{~min})$ on a hot gun $\left(\sim 250^{\circ} \mathrm{C}\right)$. Organic solutions were concentrated on rotary evaporator at $35-40{ }^{\circ} \mathrm{C}$. ${ }^{1} \mathrm{H} \mathrm{NMR}$ and ${ }^{13} \mathrm{C}$ NMR (proton-decoupled) spectra were recorded on $400 \& 500$ $\mathrm{MHz}$ NMR spectrometers in DMSO- $\mathrm{D}_{6} / \mathrm{MeOD} / \mathrm{CDCl}_{3} / \mathrm{D}_{2} \mathrm{O}$ as solvent as indicated. Chemical shifts $(\delta)$ were reported in parts per million (ppm) with respect to TMS as an internal standard. Coupling constants $(J)$ are quoted in hertz $(\mathrm{Hz})$. High Resolution Mass Spectrometry (HRMS) data were obtained by using a hybrid quadrupole-orthogonal acceleration time-of-flight system.

(R)-Methyl 2-(benzylamino)pent-4-enoate. To a stirred solution of $(R)$-methyl 2-aminopent-4-enoate $(500 \mathrm{mg}, 3.8 \mathrm{mmol}$, 1 equiv) and benzaldehyde (1.1 equiv) in $\mathrm{MeOH}(10 \mathrm{~mL})$ was added $\mathrm{NaBH}_{3} \mathrm{CN}$ (2 equiv) at $0{ }^{\circ} \mathrm{C}$ and brought to rt. After 12 hrs, the reaction mixture was quenched with $1 \mathrm{~mL}$ of water and concentrated to remove methanol. The organic layer was washed with water and brine, dried over anhydrous $\mathrm{MgSO}_{4}$, filtered, concentrated and separated by column chromatography on silica gel (eluted with EtOAc/hexanes, 1:5) to afford $800 \mathrm{mg}(90 \%)$ as a colour less liquid. $[\alpha]_{\mathrm{D}}{ }^{26}=+32.0($ c 1.0, $\left.\mathrm{CHCl}_{3}\right) ;{ }^{1} \mathrm{H} \mathrm{NMR}\left(400 \mathrm{MHz}, \mathrm{CDCl}_{3}\right): \delta 7.33-7.24(\mathrm{~m}, 5 \mathrm{H})$, 5.80-5.68 (m, $1 \mathrm{H})$, 5.17-5.04 (m, $2 \mathrm{H}), 3.88-3.63$ (m, $5 \mathrm{H})$, $3.37(\mathrm{t}, J=6.4 \mathrm{~Hz}, 1 \mathrm{H}), 2.42(\mathrm{t}, J=8.4 \mathrm{~Hz}, 2 \mathrm{H}) \mathrm{ppm}$; ${ }^{13} \mathrm{C}\left\{{ }^{1} \mathrm{H}\right\}$ NMR $\left(100 \mathrm{MHz}, \mathrm{CDCl}_{3}\right): \delta 175.1,139.7,133.6$, 129.1, 128.4, 128.3, 127.1, 118.0, 77.4, 77.0, 76.7, 60.3, 52.0, 51.7, $37.7 \mathrm{ppm} ; v_{\max }($ neat $) / \mathrm{cm}^{-1}: 3453,2950,1583,1455$, 1321, 1123, 762, $591 \mathrm{~cm}^{-1}$; HRMS (ESI) $\mathrm{m} / \mathrm{z}:[\mathrm{M}]^{+}$calcd for $\mathrm{C}_{13} \mathrm{H}_{18} \mathrm{NO}_{2}: 220.1337$; found: 220.1332 .

(R)-Methyl 2-(4-methylphenylsulfonamido)pent-4-enoate. To a stirred solution of $(R)$-methyl 2-aminopent-4-enoate (500 $\mathrm{mg}, 3.8 \mathrm{mmol}, 1$ equiv) in DCM (10 mL) was added $\mathrm{Et}_{3} \mathrm{~N}$ (1 equiv) at $0{ }^{\circ} \mathrm{C}$ and $\mathrm{TsCl}$ was added followed. After $12 \mathrm{hrs}$, the reaction mixture was quenched with $1 \mathrm{~mL}$ of water. The organic layer was washed with water and brine, dried over anhydrous $\mathrm{MgSO}_{4}$, filtered, concentrated and separated by column chromatography on silica gel (eluted with EtOAc/hexanes, $1: 5)$ to afford $910 \mathrm{mg}(83 \%)$ as a colour less liquid. $[\alpha]_{\mathrm{D}}{ }^{26}=-$ 50.0 (c 1.0, $\mathrm{CHCl}_{3}$ ); ${ }^{1} \mathrm{H} \mathrm{NMR}$ (400 MHz, $\mathrm{CDCl}_{3}$ ): $\delta$ 7.71-7.68 
(m, 2H), 7.28-7.24 (m, $2 \mathrm{H}), 5.65-5.54(\mathrm{~m}, 1 \mathrm{H}), 5.25(\mathrm{t}, J=$ 9.2 Hz, $1 \mathrm{H}), 5.09-5.02(\mathrm{~m}, 2 \mathrm{H}), 4.02-3.97$ (m, $1 \mathrm{H}), 3.49$ (s, 3 $\mathrm{H}), 2.43(\mathrm{t}, J=6.8 \mathrm{~Hz}, 1 \mathrm{H}), 2.39(\mathrm{~s}, 3 \mathrm{H}) \mathrm{ppm} ;{ }^{13} \mathrm{C}\left\{{ }^{1} \mathrm{H}\right\} \mathrm{NMR}$ $\left(100 \mathrm{MHz}, \mathrm{CDCl}_{3}\right): \delta 171.4,143.7,136.9,131.4,129.9,129.7$, 127.5, 127.3, 127.0, 119.7, 55.3, 52.4, 37.6, $21.6 \mathrm{ppm}$;

$v_{\max }($ neat $) / \mathrm{cm}^{-1}: 3466,3013,1596,1421,1335,1067,785,585$ $\mathrm{cm}^{-1}$; HRMS (ESI) $\mathrm{m} / \mathrm{z}:[\mathrm{M}]^{+}$calcd for $\mathrm{C}_{13} \mathrm{H}_{18} \mathrm{NO}_{4} \mathrm{~S} 284.0956$; found 284.0950.

(R)-3-Allylmorpholin-2-one (3): To a stirred solution of $(R)$ 2-aminopent-4-enoic acid (500 mg, $3.8 \mathrm{mmol}, 1$ equiv) and dibromoethane (1.0 equiv) in $\mathrm{CH}_{3} \mathrm{CN}(10 \mathrm{~mL})$ was added $\mathrm{K}_{2} \mathrm{CO}_{3}$ ( 2 equiv) at $0{ }^{\circ} \mathrm{C}$ and brought to reflux. After $12 \mathrm{hrs}$, the reaction mixture was cooled to RT and concentrated to remove $\mathrm{CH}_{3} \mathrm{CN}$. The organic layer was washed with water and brine, dried over anhydrous $\mathrm{MgSO}_{4}$, filtered, concentrated and separated by column chromatography on silica gel (eluted with EtOAc/hexanes, 1:1) to afford $92 \mathrm{mg}(15 \%)$ as a semi solid. $[\alpha]_{\mathrm{D}}{ }^{26}=-126.2\left(c 1.0, \mathrm{CHCl}_{3}\right) ;{ }^{1} \mathrm{H} \mathrm{NMR}\left(400 \mathrm{MHz}, \mathrm{CDCl}_{3}\right): \delta$ 5.91-5.78 (m, $1 \mathrm{H}), 5.20-5.05(\mathrm{~m}, 2 \mathrm{H}), 4.51(\mathrm{bs}, 1 \mathrm{H}), 4.45(\mathrm{t}$, $J=6.0 \mathrm{~Hz}, 1 \mathrm{H}), 3.52(\mathrm{t}, J=6.4 \mathrm{~Hz}, 2 \mathrm{H}), 2.66-2.54(\mathrm{~m}, 1 \mathrm{H})$, $2.17(\mathrm{dd}, J=0.8,6.0 \mathrm{~Hz}, 2 \mathrm{H}), 1.88(\mathrm{t}, J=6.0 \mathrm{~Hz}, 1 \mathrm{H}) \mathrm{ppm}$; ${ }^{13} \mathrm{C}\left\{{ }^{1} \mathrm{H}\right\} \mathrm{NMR}\left(100 \mathrm{MHz}, \mathrm{CDCl}_{3}\right): \delta 171.6,133.5,118.2,72.8$, 64.1, 37.2, $28.6 \mathrm{ppm} ; v_{\max }\left(\right.$ neat) $/ \mathrm{cm}^{-1}: 3302,3016,2995,1564$, 1521, 1376, 1093, 785, $643 \mathrm{~cm}^{-1}$; HRMS (ESI) $\mathrm{m} / \mathrm{z}:[\mathrm{M}]^{+} \mathrm{calcd}$ for $\mathrm{C}_{7} \mathrm{H}_{12} \mathrm{NO}_{2}$ 142.0868; found 142.0869.

$(3 R, 5 R, 6 S)$-3-allyl-5,6-diphenylmorpholin-2-one (7, Table 1, Entry 1). To a stirred solution of tert-butyl (2S,3R)-6-oxo2,3-diphenylmorpholine-4-carboxylate ${ }^{1}(300 \mathrm{mg}, 0.849 \mathrm{mmol}$, 1 equiv) and allyl iodide ( $388 \mu \mathrm{L}, 4.243 \mathrm{mmol}, 5$ equiv) in THF (5 mL) was added lithium bis(trimethylsilyl)amide (1019 $\mu \mathrm{L}, 1.019 \mathrm{mmol}, 1.2$ equiv, $1 \mathrm{M}$ solution in THF) dropwise via syringe at $-78^{\circ} \mathrm{C}$. After $40 \mathrm{~min}$, the reaction mixture was quenched with sat. $\mathrm{NH}_{4} \mathrm{Cl}$ solution. The organic layer was washed with water and brine, dried over anhydrous $\mathrm{MgSO}_{4}$, filtered and concentrated to afford $286 \mathrm{mg}(85 \%)$ as a white solid. The crude compound was diluted with DCM $(10 \mathrm{~mL})$ and added TFA $(2 \mathrm{~mL})$ at $0{ }^{\circ} \mathrm{C}$. The reaction mixture was removed from ice bath and continued stirring at RT. After $4 \mathrm{hrs,}$ the reaction mixture cooled to $0{ }^{\circ} \mathrm{C}$ and adjust $\mathrm{pH}$ (neutral) using triethylamine. The organic layer was washed with water and brine, dried over anhydrous $\mathrm{MgSO}_{4}$, filtered, concentrated, and separated by column chromatography on silica gel (eluted with EtOAc/hexanes, 2:5) to afford $199 \mathrm{mg}$ (81\% overall two steps) of 7 as a white solid. $[\alpha]_{\mathrm{D}}{ }^{26}=+260.0\left(c 1.0, \mathrm{CHCl}_{3}\right)$; M.pt. $118-120{ }^{\circ} \mathrm{C} ;{ }^{1} \mathrm{H}$ NMR $\left(400 \mathrm{MHz}, \mathrm{CDCl}_{3}\right): \delta 7.22-7.15$ (m, 6H), 7.00-6.95 (m, $2 \mathrm{H}), 6.92-6.88(\mathrm{~m}, 2 \mathrm{H})$, 5.88-5.80 (m, $1 \mathrm{H}), 5.67(\mathrm{~d}, J=3.2 \mathrm{~Hz}, 1 \mathrm{H}), 5.19-5.13(\mathrm{~m}, 2 \mathrm{H}), 4.73-4.63$ (m, 1H), 4.08-4.01 (m, 1H), 2.84-2.69 (m, 2H), 2.00 (bs, $1 \mathrm{H})$ ppm; ${ }^{13} \mathrm{C}\left\{{ }^{1} \mathrm{H}\right\}$ NMR $\left(100 \mathrm{MHz}, \mathrm{CDCl}_{3}\right): \delta 170.6,137.0$, 135.0, 133.9, 128.4, 128.2, 128.1, 127.7, 127.7, 127.2, 119.6, $85.2,77.5,77.1,76.8,56.8,56.0,37.9 \mathrm{ppm} ; v_{\max }($ neat $) / \mathrm{cm}^{-1}$ : 3331, 2981, 1700, 1456, 1196, 1036, $703 \mathrm{~cm}^{-1}$; HRMS (ESI) $m / z:[\mathrm{M}]^{+}$calcd for $\mathrm{C}_{19} \mathrm{H}_{20} \mathrm{NO}_{2} 294.1494$; found 294.1491.

(3S,5S)-3-allyl-5-phenylmorpholin-2-one (8a, Table 1, Entry 2). To a stirred solution of $(S)$-tert-butyl 2-oxo-5-phenylmorpholine-4-carboxylate ${ }^{2}(300 \mathrm{mg}, 1.08 \mathrm{mmol}, 1$ equiv) in THF $(5 \mathrm{~mL})$ and DME $(5 \mathrm{~mL})$ was added sodium bis(trimethylsilyl) amide $(594 \mu \mathrm{L}, 1.19 \mathrm{mmol}, 1.1$ equiv, $2 \mathrm{M}$ solution in THF) dropwise via syringe at $-78^{\circ} \mathrm{C}$. After $20 \mathrm{~min}$, neat allyl bromide ( $2.16 \mathrm{mmol}, 2$ equiv) was added drop wise. The reaction was quenched with sat. $\mathrm{NH}_{4} \mathrm{Cl}$ after $1.5 \mathrm{~h}$. The organic layer was washed with water and brine, dried over anhydrous $\mathrm{MgSO}_{4}$, filtered and concentrated to afford $305 \mathrm{mg}$
(90\%) as a white solid. The crude compound was diluted with DCM $(10 \mathrm{~mL})$ and added TFA $(2 \mathrm{~mL})$ at $0{ }^{\circ} \mathrm{C}$. The reaction mixture was removed from ice bath and continued stirring at RT. After $4 \mathrm{hrs}$, the reaction mixture was cooled to $0{ }^{\circ} \mathrm{C}$ and the $\mathrm{pH}$ adjusted to neutral using triethylamine. The organic layer was washed with water and brine, dried over anhydrous $\mathrm{MgSO}_{4}$, filtered, concentrated, and separated by column chromatography on silica gel (eluted with EtOAc/hexanes, 2:5) to afford $200 \mathrm{mg}$ (85\% overall two steps) of 8a as a white solid. $[\alpha]_{\mathrm{D}}^{26}=-7.6\left(c\right.$ 1.0, $\left.\mathrm{CHCl}_{3}\right) ; \mathrm{M} . \mathrm{pt} .64-66{ }^{\circ} \mathrm{C} ;{ }^{1} \mathrm{H}$ NMR $(400$ $\left.\mathrm{MHz}, \mathrm{CDCl}_{3}\right): \delta 7.50-7.31(\mathrm{~m}, 5 \mathrm{H}), 5.88-5.80(\mathrm{~m}, 1 \mathrm{H}), 5.24-$ 5.15 (m, $2 \mathrm{H}), 4.44-4.28$ (m, $3 \mathrm{H}), 3.86(\mathrm{dd}, J=3.6 \mathrm{~Hz}, 1 \mathrm{H})$, 2.72-2.62 (m, 2H), 1.97 (bs, $1 \mathrm{H}) \mathrm{ppm} ;{ }^{13} \mathrm{C}\left\{{ }^{1} \mathrm{H}\right\}$ NMR (100 $\left.\mathrm{MHz}, \mathrm{CDCl}_{3}\right): \delta 171.0,133.6,129.0,126.9,119.7,77.3,77.1$, 76.8, 73.2, 54.7, 53.1, $36.4 \mathrm{ppm} ; v_{\max }$ (neat) $/ \mathrm{cm}^{-1}: 3323,2992$, 1733, 1464, 1182, 1025, $698 \mathrm{~cm}^{-1}$; HRMS (ESI) $\mathrm{m} / \mathrm{z}:[\mathrm{M}]^{+}$ calcd for $\mathrm{C}_{13} \mathrm{H}_{16} \mathrm{NO}_{2} 218.1176$; found 218.1175.

(3S,5S)-5-Phenyl-3-(prop-2-ynyl) morpholin-2-one (8b, Table 1, Entry 3). To a stirred solution of $(S)$-tert-butyl 2-oxo-5phenylmorpholine-4-carboxylate ${ }^{2}(300 \mathrm{mg}, 1.08 \mathrm{mmol}, 1$ equiv) in THF $(5 \mathrm{~mL})$ and DME $(5 \mathrm{~mL})$ was added sodium bis(trimethylsilyl) amide (594 $\mu \mathrm{L}, 1.19 \mathrm{mmol}, 1.1$ equiv, $2 \mathrm{M}$ solution in THF) dropwise via syringe at $-78^{\circ} \mathrm{C}$. After 20 min, $80 \%$ propargyl bromide ( $2.16 \mathrm{mmol}, 2$ equiv) in toluene was added drop wise. The reaction was quenched with sat. $\mathrm{NH}_{4} \mathrm{Cl}$ after $1.5 \mathrm{~h}$. The organic layer was washed with water and brine, dried over anhydrous $\mathrm{MgSO}_{4}$, filtered and concentrated to afford $315 \mathrm{mg}$ as a semi solid. The crude compound was diluted with DCM $(10 \mathrm{~mL})$ and added TFA $(2 \mathrm{~mL})$ at 0 ${ }^{\circ} \mathrm{C}$. The reaction mixture was removed from ice bath and continued stirring at RT. After $4 \mathrm{hrs}$, the reaction mixture cooled to $0{ }^{\circ} \mathrm{C}$ and adjust $\mathrm{pH}$ (neutral) using triethylamine. The organic layer was washed with water and brine, dried over anhydrous $\mathrm{MgSO}_{4}$, filtered, concentrated, and separated by column chromatography on silica gel (eluted with EtOAc/hexanes, $2: 5)$ to afford $210 \mathrm{mg}$ ( $85 \%$ overall two steps) of $\mathbf{8 b}$ as a white solid. $[\alpha]_{\mathrm{D}}{ }^{26}=+152.0\left(\right.$ c 1.0, $\left.\mathrm{CHCl}_{3}\right)$; M.pt. $96-98{ }^{\circ} \mathrm{C} ;{ }^{1} \mathrm{H}$ NMR (400 MHz, $\left.\mathrm{CDCl}_{3}\right)$ : $\delta$ 7.48-7.30 (m, 5H), 4.49-4.28 (m, $3 \mathrm{H}), 4.01-3.92(\mathrm{~m}, 1 \mathrm{H}), 2.91-2.78(\mathrm{~m}, 2 \mathrm{H}), 2.38$ (bs, $1 \mathrm{H})$, $2.08(\mathrm{~s}, 1 \mathrm{H}) \mathrm{ppm} ;{ }^{13} \mathrm{C}\left\{{ }^{1} \mathrm{H}\right\} \mathrm{NMR}\left(100 \mathrm{MHz}, \mathrm{CDCl}_{3}\right): \delta 169.7$, 138.1, 129.1, 128.6, 127.1, 77.4, 77.1, 76.8, 73.5, 72.1, 54.3, 53.1, $22.5 \mathrm{ppm} ; v_{\max }($ neat $) / \mathrm{cm}^{-1}: 2970,2890,1603,1456$, $1314,1225,1039,757,646 \mathrm{~cm}^{-1}$; HRMS (ESI) $\mathrm{m} / \mathrm{z}:[\mathrm{M}]^{+}$calcd for $\mathrm{C}_{13} \mathrm{H}_{14} \mathrm{NO}_{2} 216.1025$; found 216.1029.

(3S,5S)-3-((E)-But-2-enyl)-5-phenylmorpholin-2-one (8c, Table 1, Entry 4). To a stirred solution of (S)-tert-butyl 2oxo-5-phenylmorpholine-4-carboxylate ${ }^{2}(300 \mathrm{mg}, 1.08 \mathrm{mmol}$, 1 equiv) in THF ( $5 \mathrm{~mL})$ and DME $(5 \mathrm{~mL})$ was added sodium bis(trimethylsilyl) amide (594 $\mu \mathrm{L}, 1.19 \mathrm{mmol}, 1.1$ equiv, $2 \mathrm{M}$ solution in THF) dropwise via syringe at $-78^{\circ} \mathrm{C}$. After 20 min, neat crotyl bromide ( $2.16 \mathrm{mmol}, 2$ equiv) was added drop wise. The reaction was quenched with sat. $\mathrm{NH}_{4} \mathrm{Cl}$ after $1.5 \mathrm{~h}$. The organic layer was washed with water and brine, dried over anhydrous $\mathrm{MgSO}_{4}$, filtered and concentrated to afford $315 \mathrm{mg}$ as a white solid. The crude compound was diluted with DCM $(10 \mathrm{~mL})$ and added TFA $(2 \mathrm{~mL})$ at $0{ }^{\circ} \mathrm{C}$. The reaction mixture was removed from ice bath and continued stirring at RT. After $4 \mathrm{hrs}$, the reaction mixture cooled to $0{ }^{\circ} \mathrm{C}$ and adjust $\mathrm{pH}$ (neutral) using triethylamine. The organic layer was washed with water and brine, dried over anhydrous $\mathrm{MgSO}_{4}$, filtered, concentrated, and separated by column chromatography on silica gel (eluted with EtOAc/hexanes, 2:5) to afford $216 \mathrm{mg}(86 \%$ overall two steps) of $\mathbf{8 c}$ as a white solid. $[\alpha]_{\mathrm{D}}^{26}=+82.0(c$ 1.0, 
$\left.\mathrm{CHCl}_{3}\right)$; M.pt. $82-84{ }^{\circ} \mathrm{C} ;{ }^{1} \mathrm{H}$ NMR (400 MHz, $\left.\mathrm{CDCl}_{3}\right): \delta 7.48-$ 7.29 (m, 5H), 5.66-5.43 (m, $2 \mathrm{H}), 4.41-4.23$ (m, $3 \mathrm{H}), 3.84-$ $3.74(\mathrm{~m}, 1 \mathrm{H}), 2.63-2.53(\mathrm{~m}, 2 \mathrm{H}), 1.98$ (bs, $1 \mathrm{H}), 1.69-1.63$ (m, $2 \mathrm{H}) \mathrm{ppm} ;{ }^{13} \mathrm{C}\left\{{ }^{1} \mathrm{H}\right\} \mathrm{NMR}\left(100 \mathrm{MHz}, \mathrm{CDCl}_{3}\right): \delta 171.2$, 138.6, 130.5, 128.9, 128.5, 127.1, 126.0, 77.5, 77.2, 76.8, 73.2, 55.1, 53.1, 35.2, $18.1 \mathrm{ppm} ; v_{\max }$ (neat) $/ \mathrm{cm}^{-1}: 2981,1603$, 1453, 1164, 912, $647 \mathrm{~cm}^{-1}$; HRMS (ESI) $\mathrm{m} / z:[\mathrm{M}]^{+}$calcd for $\mathrm{C}_{14} \mathrm{H}_{18} \mathrm{NO}_{2} 232.1338$; found 232.1337.

(3S,5S)-3-(2-Methylallyl)-5-phenylmorpholin-2-one (8d, Table 1, Entry 5). To a stirred solution of (S)-tert-butyl 2oxo-5-phenylmorpholine-4-carboxylate ${ }^{2}(300 \mathrm{mg}, 1.08 \mathrm{mmol}$, 1 equiv) in THF ( $5 \mathrm{~mL})$ and DME $(5 \mathrm{~mL})$ was added sodium bis(trimethylsi1yl) amide $(594 \mu \mathrm{L}, 1.19 \mathrm{mmol}, 1.1$ equiv, $2 \mathrm{M}$ solution in THF) dropwise via syringe at $-78^{\circ} \mathrm{C}$. After 20 min, neat 3-bromo-2-methylprop-1-ene ( $2.16 \mathrm{mmol}, 2$ equiv) was added drop wise. The reaction was quenched with sat. $\mathrm{NH}_{4} \mathrm{Cl}$ after $1.5 \mathrm{~h}$. The organic layer was washed with water and brine, dried over anhydrous $\mathrm{MgSO}_{4}$, filtered and concentrated to afford $315 \mathrm{mg}$ as a white solid. The crude compound was diluted with DCM $(10 \mathrm{~mL})$ and added TFA $(2 \mathrm{~mL})$ at 0 ${ }^{\circ} \mathrm{C}$. The reaction mixture was removed from ice bath and continued stirring at RT. After $4 \mathrm{hrs}$, the reaction mixture cooled to $0{ }^{\circ} \mathrm{C}$ and adjust $\mathrm{pH}$ (neutral) using triethylamine. The organic layer was washed with water and brine, dried over anhydrous $\mathrm{MgSO}_{4}$, filtered, concentrated, and separated by column chromatography on silica gel (eluted with EtOAc/hexanes, $2: 5)$ to afford $225 \mathrm{mg}$ (90\% overall two steps) of $\mathbf{8 d}$ as a solid. $[\alpha]_{\mathrm{D}}{ }^{26}=-106.8\left(c 1.0, \mathrm{CHCl}_{3}\right) ;$ M.pt. $92-94^{\circ} \mathrm{C} ;[\alpha]_{\mathrm{D}}{ }^{25}-12.3$ (c 1.0, $\left.\mathrm{CHCl}_{3}\right) ;{ }^{1} \mathrm{H}$ NMR $\left(400 \mathrm{MHz}, \mathrm{CDCl}_{3}\right): \delta 7.43-7.31(\mathrm{~m}$, 5H), 4.92-4.81 (m, $1 \mathrm{H}), 4.80-4.75(\mathrm{~m}, 1 \mathrm{H}), 4.36-4.27$ (m, 3 H), 3.97-3.94 (m, $1 \mathrm{H}), 2.66-2.59$ (m, $1 \mathrm{H}), 1.99$ (bs, $1 \mathrm{H})$, 1.75 (s, $3 \mathrm{H}) \mathrm{ppm} ;{ }^{13} \mathrm{C}\left\{{ }^{1} \mathrm{H}\right\} \mathrm{NMR}\left(100 \mathrm{MHz}, \mathrm{CDCl}_{3}\right): \delta 171.1$, 141.1, 138.1, 128.9, 128.6, 127.2, 115.2, 77.43, 77.1, 76.8, 73.9, 53.6, 52.4, 40.4, $21.6 \mathrm{ppm} ; v_{\max }($ neat $) / \mathrm{cm}^{-1}: 2971,1648$, $1455,1216,1039,898,596 \mathrm{~cm}^{-1}$; HRMS (ESI) $\mathrm{m} / \mathrm{z}:[\mathrm{M}]^{+}$calcd for $\mathrm{C}_{14} \mathrm{H}_{18} \mathrm{NO}_{2} 232.1338$; found 232.1338.

(3S,5S)-3-(3-Methylbut-2-enyl)-5-phenylmorpholin-2-one (8e, Table 1, Entry 6). To a stirred solution of (S)-tert-butyl 2-oxo-5-phenylmorpholine-4-carboxylate ${ }^{2}(300 \mathrm{mg}, 1.08$ mmol, 1 equiv) in THF $(5 \mathrm{~mL})$ and DME $(5 \mathrm{~mL})$ was added sodium bis(trimethylsilyl) amide $(594 \mu \mathrm{L}, 1.19 \mathrm{mmol}, 1.1$ equiv, $2 \mathrm{M}$ solution in THF) dropwise via syringe at $-78^{\circ} \mathrm{C}$. After $20 \mathrm{~min}$, neat 1-bromo-3-methylbut-2-ene $(2.16 \mathrm{mmol}, 2$ equiv) was added drop wise. The reaction was quenched with sat. $\mathrm{NH}_{4} \mathrm{Cl}$ after $1.5 \mathrm{~h}$. The organic layer was washed with water and brine, dried over anhydrous $\mathrm{MgSO}_{4}$, filtered and concentrated to afford $315 \mathrm{mg}$ as a white solid. The crude compound was diluted with DCM $(10 \mathrm{~mL})$ and added TFA (2 $\mathrm{mL})$ at $0{ }^{\circ} \mathrm{C}$. The reaction mixture was removed from ice bath and continued stirring at RT. After $4 \mathrm{hrs}$, the reaction mixture cooled to $0{ }^{\circ} \mathrm{C}$ and adjust $\mathrm{pH}$ (neutral) using triethylamine. The organic layer was washed with water and brine, dried over anhydrous $\mathrm{MgSO}_{4}$, filtered, concentrated, and separated by column chromatography on silica gel (eluted with EtOAc/hexanes, 2:5) to afford $212 \mathrm{mg}(80 \%$ overall two steps) of $\mathbf{8 e}$ as a solid. $[\alpha]_{\mathrm{D}}{ }^{26}=-263.2\left(c 1.0, \mathrm{CHCl}_{3}\right)$; M.pt. $96-98{ }^{\circ} \mathrm{C} ;{ }^{1} \mathrm{H}$ NMR (400 MHz, $\left.\mathrm{CDCl}_{3}\right): \delta 7.43-7.30(\mathrm{~m}, 5 \mathrm{H}), 5.20-5.12(\mathrm{~m}$, $1 \mathrm{H}), 4.92-4.81(\mathrm{~m}, 1 \mathrm{H}), 4.80-4.75(\mathrm{~m}, 1 \mathrm{H}), 4.36-4.27$ (m, 3 H), 3.97-3.94 (m, $1 \mathrm{H}), 3.83-3.77(\mathrm{~m}, 1 \mathrm{H}), 2.74-2.51(\mathrm{~m}, 2$ $\mathrm{H}), 1.91$ (bs, $1 \mathrm{H}), 1.71$ (s, $3 \mathrm{H}), 1.64$ (s, $3 \mathrm{H}) \mathrm{ppm} ;{ }^{13} \mathrm{C}\left\{{ }^{1} \mathrm{H}\right\}$ NMR (100 MHz, $\left.\mathrm{CDCl}_{3}\right): \delta 171.3,138.5,136.9,128.9,128.5$, 127.1, 119.3, 77.4, 77.1, 76.8, 73.5, 55.8, 53.2, 30.8, 25.9, $18.1 \mathrm{ppm} ; v_{\max }\left(\right.$ neat) $/ \mathrm{cm}^{-1}: 2971,1746,1387,1174,1050,697$,
$607 \mathrm{~cm}^{-1}$; HRMS (ESI) m/z: [M] ${ }^{+}$calcd for $\mathrm{C}_{15} \mathrm{H}_{20} \mathrm{NO}_{2}$ 246.1494; found 246.1498.

General procedure A: the TFA mediated aza-Prins reaction: A round-bottomed flask was charged with aldehyde $(0.5$ eq.) and dichloromethane $(10 \mathrm{~mL})$. To the resulting suspension was added neat TFA ( $1 \mathrm{mmol}, 2$ eq.). After stirring the mixture for $15 \mathrm{~min}$ at room temperature, corresponding amine derivative $(0.5 \mathrm{mmol}, 1.00$ eq.) in dichloromethane $(3 \mathrm{~mL})$ was added and the resulting mixture stirred for $12 \mathrm{hrs}$. After this time, the solvent was concentrated completely. The resulting material was diluted with methanol $\left(15 \mathrm{~mL}\right.$ ) and $\mathrm{K}_{2} \mathrm{CO}_{3}$ (excess, 5 equiv.) added and stirred for 3 hrs. The solvent was removed and diluted with DCM. The organic layer was separated and the aqueous layer was extracted with dichloromethane $(2$ X $10 \mathrm{~mL})$. The combined organic layers were dried over magnesium sulfate, filtered and concentrated in vacuo, and purified by chromatography (silica gel 100-200 mesh, 30:70 EtOAc/Hexane).

$(3 S, 4 R, 6 S, 8 R, 9 a R)$-8-hydroxy-3,4-diphenyl-6-propylhexahydropyrido[2,1-c] [1,4] oxazin-1(6H)-one (9a, Table 2 Entry 1). Following the general procedure, $\mathbf{A}$, the title compound 9a was prepared from compound $7 \mathbf{a}(146 \mathrm{mg})$ and butanal as a white solid $(138 \mathrm{mg}, 76 \%)$. $[\alpha]_{\mathrm{D}}{ }^{26}=-40.0\left(\right.$ c $\left.1.0, \mathrm{CHCl}_{3}\right)$; M.pt. $116-118{ }^{\circ} \mathrm{C} ;{ }^{1} \mathrm{H}$ NMR $\left(400 \mathrm{MHz}, \mathrm{CDCl}_{3}\right): \delta$ 7.22-7.20 (m, 2 H), 7.18-7.13 (m, 5 H), 7.10-7.05 (m, 2 H), 6.96-6.91 $(\mathrm{m}, 2 \mathrm{H}), 6.79-6.74(\mathrm{~m}, 2 \mathrm{H}), 5.53(\mathrm{~d}, J=4.4 \mathrm{~Hz}, 1 \mathrm{H}), 4.59$ $(\mathrm{d}, J=4.4 \mathrm{~Hz}, 1 \mathrm{H}), 4.17$ (dd, $J=3.6,8.8 \mathrm{~Hz}, 1 \mathrm{H}), 4.05$ (sep, $J=6.4 \mathrm{~Hz}, 1 \mathrm{H}), 3.04-2.96$ (m, 1H), 2.30-2.23 (m, $1 \mathrm{H}), 2.09$ $1.94(\mathrm{~m}, 1 \mathrm{H}), 1.58-1.40(\mathrm{~m}, 6 \mathrm{H}), 1.34-1.19(\mathrm{~m}, 3 \mathrm{H}), 1.15-$ $1.10(\mathrm{~m}, 1 \mathrm{H}), 0.78(\mathrm{t}, J=7.2 \mathrm{~Hz}, 3 \mathrm{H}) \mathrm{ppm} ;{ }^{13} \mathrm{C}\left\{{ }^{1} \mathrm{H}\right\} \mathrm{NMR}$ $\left(100 \mathrm{MHz} \mathrm{CDCl}_{3}\right): \delta 170.9,135.7,135.5,129.8,128.3,128.1$, 128.0, 127.9, 127.6, 83.7, 77.4, 77.2, 77.0, 76.7, 64.9, 61.9, $54.5,54.2,34.6,33.5,33.2,19.9,14.1 \mathrm{ppm} ; v_{\max }($ neat $) / \mathrm{cm}^{-1}$ : 2981, 1730, 1355, 1044, 921, $655 \mathrm{~cm}^{-1}$; HRMS (ESI) $\mathrm{m} / \mathrm{z}$ : $[\mathrm{M}]+$ calcd for $\mathrm{C}_{23} \mathrm{H}_{28} \mathrm{O}_{3} \mathrm{~N}: 366.2064$; found: 366.2064. Compound 9a was also prepared using the same method starting from 7a (146 mg) and butanal diethyl acetal $(73 \mathrm{mg})$ in $132 \mathrm{mg}$, 73\% yield (Table 2 Entry 11). All data was in agreement with that presented above.

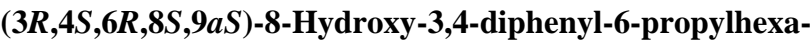
hydropyrido[2,1-c] [1,4] oxazin-1(6H)-one (9a', Table 2,

Entry 2). Following the general procedure, $\mathbf{A}$, the title compound 9a' was prepared from compound $7 \mathbf{b}$ (146 mg) (opposite isomer of 7a) and butanal as a white solid (150 mg, $82 \%)$. $[\alpha]_{\mathrm{D}}{ }^{26}=+360.00$ (c 1.0, MeOH); M.pt. 124-126 oC; $1 \mathrm{H} \mathrm{NMR}$ (400 MHz, $\left.\mathrm{CDCl}_{3}\right): \delta$ 7.22-7.20 (m, $\left.2 \mathrm{H}\right), 7.18-7.13(\mathrm{~m}, 5 \mathrm{H})$, 7.10-7.05 (m, $2 \mathrm{H}), 6.96-6.91$ (m, $2 \mathrm{H}), 6.79-6.74$ (m, $2 \mathrm{H})$, $5.53(\mathrm{~d}, J=4.4 \mathrm{~Hz}, 1 \mathrm{H}), 4.59(\mathrm{~d}, J=4.4 \mathrm{~Hz}, 1 \mathrm{H}), 4.17(\mathrm{dd}, J$ = 3.6, 8.8 Hz, 1 H), $4.05(\mathrm{sep}, J=6.4 \mathrm{~Hz}, 1 \mathrm{H}), 3.04-2.96(\mathrm{~m}$, $1 \mathrm{H}), 2.30-2.23(\mathrm{~m}, 1 \mathrm{H}), 2.09-1.94(\mathrm{~m}, 1 \mathrm{H}), 1.58-1.40(\mathrm{~m}, 6$ $\mathrm{H}), 1.34-1.19(\mathrm{~m}, 3 \mathrm{H}), 1.15-1.10(\mathrm{~m}, 1 \mathrm{H}), 0.78(\mathrm{t}, J=7.2$ $\mathrm{Hz}, 3 \mathrm{H}) \mathrm{ppm} ;{ }^{13} \mathrm{C}\left\{{ }^{1} \mathrm{H}\right\} \mathrm{NMR}\left(100 \mathrm{MHz}, \mathrm{CDCl}_{3}\right): \delta 171.0$, 135.7, 135.5, 129.9, 128.3, 128.1, 127.9, 127.6, 83.8, 77.4, 77.1, 76.8, 64.9, 61.8, 54.5, 54.3, 34.6, 33.5, 33.2, 19.9, 14.2 ppm; $v_{\max }($ neat $) / \mathrm{cm}^{-1}: 2981,1767,1455,1130,1042,753,658$ $\mathrm{cm}^{-1}$; HRMS (ESI) m/z: [M] $]^{+}$calcd for $\mathrm{C}_{23} \mathrm{H}_{28} \mathrm{O}_{3} \mathrm{~N}$ 366.2064; found 366.2064.

(3S,4R,6S,8R,9aR)-8-hydroxy-6-phenethyl-3,4-diphenylhexahydropyrido[2,1-c] [1,4] oxazin-1(6H)-one (9b, Table 2 Entry 3). Following the general procedure, A, the title compound $9 \mathrm{~b}$ was prepared from compound $7 \mathbf{a}(146 \mathrm{mg})$ and 3 phenyl propionaldehyde as a white solid $(173 \mathrm{mg}, 81 \%)$. $[\alpha]_{\mathrm{D}}^{26}=-421.0\left(c\right.$ 1.0, $\left.\mathrm{CHCl}_{3}\right)$; M.pt. $136-138{ }^{\circ} \mathrm{C} ;{ }^{1} \mathrm{H}$ NMR 
(400 MHz, $\left.\mathrm{CDCl}_{3}\right): \delta$ 7.27-7.09 (m, $\left.7 \mathrm{H}\right), 7.04(\mathrm{t}, J=7.2 \mathrm{~Hz}, 2$ H), $6.94(\mathrm{~d}, J=7.2 \mathrm{~Hz}, 4 \mathrm{H}), 6.73(\mathrm{~d}, J=8.0 \mathrm{~Hz}, 2 \mathrm{H}), 5.55$ $(\mathrm{d}, J=4.0 \mathrm{~Hz}, 1 \mathrm{H}), 4.52(\mathrm{dd}, J=4.0,14.4 \mathrm{~Hz}, 2 \mathrm{H}), 4.20(\mathrm{dd}$, $J=2.4,10 \mathrm{~Hz}, 1 \mathrm{H}), 4.01-3.92(\mathrm{~m}, 1 \mathrm{H}), 3.01-2.94(\mathrm{~m}, 1 \mathrm{H})$, 2.90 (bs, $1 \mathrm{H}), 2.66-2.53$ (m, $1 \mathrm{H}), 2.38-2.20$ (m, 2 H), 2.021.77 (m, $2 \mathrm{H}), 1.62-1.39$ (m, $3 \mathrm{H}) \mathrm{ppm} ;{ }^{13} \mathrm{C}\left\{{ }^{1} \mathrm{H}\right\}$ NMR $(100$ $\left.\mathrm{MHz}, \mathrm{CDCl}_{3}\right): \delta 170.7,141.6,135.6,135.5,129.6,128.1$, 128.1, 128.0, 128.0, 127.8, 127.6, 127.3, 125.6, 83.4, 77.9, 77.6, 77.2, 63.6, 62.0, 54.6, 54.3, 34.8, 33.2, 32.7, 32.6 ppm; $v_{\max }($ neat $) / \mathrm{cm}^{-1}: 2933,1738,1626,1221,953,732 \mathrm{~cm}^{-1}$; HRMS (ESI) $m / z$ : [M] $]^{+}$calcd for $\mathrm{C}_{28} \mathrm{H}_{30} \mathrm{O}_{3} \mathrm{~N}$ 428.2220; found 428.2219 .

$(3 S, 4 R, 6 S, 8 R, 9 a R)-8$-hydroxy-3,4-diphenyl-6-undecylhexahydropyrido[2,1-c] $[1,4]$ oxazin-1 $(6 H)$-one (9c, Table 2 Entry 4). Following the general procedure, $\mathbf{A}$, the title compound 9c was prepared from compound $7 \mathbf{a}(146 \mathrm{mg})$ and dodecanal as a white solid $(179 \mathrm{mg}, 75 \%) .[\alpha]_{\mathrm{D}}{ }^{26}=+78.0\left(c 1.0, \mathrm{CHCl}_{3}\right)$; M.pt. $126-128{ }^{\circ} \mathrm{C} ;{ }^{1} \mathrm{H} \mathrm{NMR}\left(400 \mathrm{MHz}, \mathrm{CDCl}_{3}\right): \delta 7.23-7.13$ (m, $4 \mathrm{H}), 7.10-7.05(\mathrm{~m}, 2 \mathrm{H}), 6.96-6.91(\mathrm{~m}, 2 \mathrm{H}), 6.77-6.73$ $(\mathrm{m}, 2 \mathrm{H}), 5.54(\mathrm{~d}, J=4.0 \mathrm{~Hz}, 1 \mathrm{H}), 4.57(\mathrm{~d}, J=4.0 \mathrm{~Hz}, 1 \mathrm{H})$, 4.17 (dd, $J=3.2,8.8 \mathrm{~Hz}, 1 \mathrm{H}), 4.04$ (sep, $J=6.0 \mathrm{~Hz}, 1 \mathrm{H}$ ), 3.02-2.93 (m, 1H), 2.32-2.24 (m, $1 \mathrm{H}), 2.03-1.93(\mathrm{~m}, 1 \mathrm{H})$, $1.61-1.38(\mathrm{~m}, 3 \mathrm{H}), 1.33-1.02(\mathrm{~m}, 22 \mathrm{H}), 0.86(\mathrm{t}, J=6.8 \mathrm{~Hz}, 3$ H) ppm; ${ }^{13} \mathrm{C}\left\{{ }^{1} \mathrm{H}\right\}$ NMR $\left(100 \mathrm{MHz}, \mathrm{CDCl}_{3}\right): \delta 170.9,135.7$, 135.5, 129.9, 128.3, 128.1, 127.9, 127.5, 83.7, 77.4, 77.0, 76.7, 64.9, 61.9, 54.6, 54.2, 34.6, 33.3, 32.0, 31.2, 29.7, 29.6, 29.4, 26.6, 22.7, $14.2 \mathrm{ppm} ; v_{\max }$ (neat) $/ \mathrm{cm}^{-1}: 2971,1712,1457$, $1152,885,730 \mathrm{~cm}^{-1}$; HRMS (ESI) $\mathrm{m} / z:[\mathrm{M}]^{+}$calcd for $\mathrm{C}_{31} \mathrm{H}_{44}$ $\mathrm{O}_{3} \mathrm{~N}$ 478.3316; found 478.3309 .

$(3 S, 4 R, 6 S, 8 R, 9 a R)-8$-hydroxy-6-nonyl-3,4-diphenylhexahydropyrido[2,1-c] $[1,4]$ oxazin-1 $(6 H)$-one (9d, Table 2 Entry 5). Following the general procedure, $\mathbf{A}$, the title compound $\mathbf{9 d}$ was prepared from compound $7 \mathbf{a}(146 \mathrm{mg})$ and decanal as a white solid (164 mg, 73\%). $[\alpha]_{\mathrm{D}}^{26}=-52.0\left(c 1.0, \mathrm{CHCl}_{3}\right)$; M.pt. $124-126{ }^{\circ} \mathrm{C} ;{ }^{1} \mathrm{H}$ NMR (400 MHz, $\left.\mathrm{CDCl}_{3}\right): \delta 7.23-7.13$ (m, 4 H), 7.10-7.04 (m, 2 H), 6.96-6.90 (m, 2 H), 6.77-6.72 $(\mathrm{m}, 2 \mathrm{H}), 5.52(\mathrm{~d}, J=4.0 \mathrm{~Hz}, 1 \mathrm{H}), 4.57(\mathrm{~d}, J=4.0 \mathrm{~Hz}, 1 \mathrm{H})$, 4.17 (dd, $J=3.2,8.8 \mathrm{~Hz}, 1 \mathrm{H}), 4.09-4.00$ (m, $1 \mathrm{H}), 3.00-2.93$ $(\mathrm{m}, 1 \mathrm{H}), 2.30-2.24(\mathrm{~m}, 1 \mathrm{H}), 2.02-1.93(\mathrm{~m}, 1 \mathrm{H}), 1.46-1.38(\mathrm{~m}$, $1 \mathrm{H}), 1.35-1.06(\mathrm{~m}, 15 \mathrm{H}), 0.86(\mathrm{t}, J=6.8 \mathrm{~Hz}, 3 \mathrm{H}) \mathrm{ppm}$; ${ }^{13} \mathrm{C}\left\{{ }^{1} \mathrm{H}\right\}$ NMR $\left(100 \mathrm{MHz}, \mathrm{CDCl}_{3}\right): \delta 170.9,135.7,135.5$, 129.9, 128.3, 128.1, 127.9, 127.5, 83.7, 77.4, 77.1, 76.8, 64.9, 61.9, 54.6, 54.2, 34.6, 33.3, 31.9, 31.2, 29.6, 29.3, 26.7, 22.8, $14.2 \mathrm{ppm} ; v_{\max }($ neat $) / \mathrm{cm}^{-1}: 2983,1738,1454,1224,703 \mathrm{~cm}^{-1}$; HRMS (ESI) $m / z$ : [M] $]^{+}$calcd for $\mathrm{C}_{29} \mathrm{H}_{40} \mathrm{O}_{3} \mathrm{~N} 450.3003$; found 450.3001 .

(3S,4R,6S,8R,9aR)-6-heptyl-8-hydroxy-3,4-diphenylhexahydropyrido[2,1-c] $[1,4]$ oxazin-1(6H)-one (9e, Table 2 Entry 6). Following the general procedure, $\mathbf{A}$, the title compound was prepared from compound $\mathbf{5}(146 \mathrm{mg})$ and octanal as a white solid (151 mg, $72 \%)$. $[\alpha]_{\mathrm{D}}{ }^{26}=+124.0\left(\right.$ c 1.0, $\left.\mathrm{CHCl}_{3}\right)$; M.pt. $108-110{ }^{\circ} \mathrm{C} ;{ }^{1} \mathrm{H}$ NMR (400 MHz, $\left.\mathrm{CDCl}_{3}\right): \delta 7.22-7.13$ (m, $4 \mathrm{H}), 7.07$ (t, $J=8 \mathrm{~Hz}, 2 \mathrm{H}), 6.97-6.93$ (m, $2 \mathrm{H}), 6.79-6.73$ $(\mathrm{m}, 2 \mathrm{H}), 5.53(\mathrm{~d}, J=4.0 \mathrm{~Hz}, 1 \mathrm{H}), 4.57(\mathrm{~d}, J=4.0 \mathrm{~Hz}, 1 \mathrm{H})$, $4.17(\mathrm{dd}, J=3.2,8.8 \mathrm{~Hz}, 1 \mathrm{H}), 4.04(\mathrm{sep}, J=6.0 \mathrm{~Hz}, 1 \mathrm{H})$, 3.02-2.94 (m, 1H), 2.31-2.22 (m, $1 \mathrm{H}), 2.03-1.93(\mathrm{~m}, 1 \mathrm{H})$, 1.87 (bs, $1 \mathrm{H}), 1.58-1.38(\mathrm{~m}, 3 \mathrm{H}), 1.32-1.05(\mathrm{~m}, 12 \mathrm{H}), 0.86$ $(\mathrm{t}, J=6.8 \mathrm{~Hz}, 3 \mathrm{H}) \mathrm{ppm} ;{ }^{13} \mathrm{C}\left\{{ }^{1} \mathrm{H}\right\} \mathrm{NMR}\left(100 \mathrm{MHz}, \mathrm{CDCl}_{3}\right): \delta$ 171.0, 135.7, 135.5, 129.9, 128.3, 128.1, 127.9, 127.6, 83.8, 77.4, 77.3, 77.1, 76.8, 64.8, 61.9, 54.6, 54.2, 34.6, 33.3, 31.8, $31.2,29.5,29.3,26.7,22.7,14.1 \mathrm{ppm} ; v_{\max }($ neat $) / \mathrm{cm}^{-1}: 2981$, 1740, 1382, 1071, 956, $696 \mathrm{~cm}^{-1}$; HRMS (ESI) $\mathrm{m} / \mathrm{z}:[\mathrm{M}]^{+} \mathrm{calcd}$ for $\mathrm{C}_{27} \mathrm{H}_{36} \mathrm{O}_{3} \mathrm{~N}$ 422.2690; found 422.2688.
$(3 S, 4 R, 6 R, 8 R, 9 a R)$-6-benzhydryl-8-hydroxy-3,4-diphenylhexahydropyrido[2,1-c] $[1,4]$ oxazin-1 $(6 H)$-one (9f, Table 2 Entry 7). Following the general procedure, A, the title compound $9 \mathbf{e}$ was prepared from compound $7 \mathbf{a}(146 \mathrm{mg})$ and diphenyl acetaldehyde as a white solid $(191 \mathrm{mg}, 78 \%) .[\alpha]_{\mathrm{D}}{ }^{26}=$ +125.0 (c 1.0, $\mathrm{CHCl}_{3}$ ); M.pt. 140-142 ${ }^{\circ} \mathrm{C}$; ${ }^{1} \mathrm{H} \mathrm{NMR}(400 \mathrm{MHz}$, $\left.\mathrm{CDCl}_{3}\right): \delta$ 7.21-7.01 (m, $\left.14 \mathrm{H}\right), 6.93-6.85(\mathrm{~m}, 2 \mathrm{H}), 6.53-6.49$ (m, $4 \mathrm{H}), 5.32(\mathrm{~d}, J=4.0 \mathrm{~Hz}, 1 \mathrm{H}), 4.65(\mathrm{~d}, J=4.0 \mathrm{~Hz}, 1 \mathrm{H})$, $4.46(\mathrm{dd}, J=4.0 \mathrm{~Hz}, 1 \mathrm{H}), 4.16(\mathrm{~d}, J=12.0 \mathrm{~Hz}, 1 \mathrm{H}), 3.76-$ $3.73(\mathrm{~m}, 1 \mathrm{H}), 2.41-2.32(\mathrm{~m}, 1 \mathrm{H}), 2.14-2.03(\mathrm{~m}, 1 \mathrm{H}), 1.58-$ $1.51(\mathrm{~m}, 1 \mathrm{H}), 1.41-1.32(\mathrm{~m}, 1 \mathrm{H}) \mathrm{ppm} ;{ }^{13} \mathrm{C}\left\{{ }^{1} \mathrm{H}\right\} \mathrm{NMR}(100$ $\left.\mathrm{MHz}, \mathrm{CDCl}_{3}\right): \delta 170.1,142.5,141.9,135.3,134.3,130.3$, 129.0, 128.5, 128.3, 127.8, 127.7, 127.7, 126.9, 126.8, 84.1, 77.4, 77.1, 76.7, 63.9, 61.8, 58.3, 54.8, 52.7, 33.4, 29.3 ppm; $v_{\max }($ neat $) / \mathrm{cm}^{-1}: 2981,1726,1382,1073,957,695 \mathrm{~cm}^{-1}$; HRMS (ESI) $m / z$ : [M] $]^{+}$calcd for $\mathrm{C}_{33} \mathrm{H}_{32} \mathrm{O}_{3} \mathrm{~N} 490.2377$; found 490.2369 .

$(3 S, 4 R, 6 R, 8 R, 9 a R)$-Ethyl 8-hydroxy-1-oxo-3,4-diphenyloctahydropyrido[2,1-c] [1,4] oxazine-6-carboxylate $(9 \mathrm{~g}$, Table 2 Entry 8). Following the general procedure, $\mathbf{A}$, the $t i$ tle compound was prepared from compound $7 \mathbf{a}(146 \mathrm{mg})$ and ethyl glyoxylate $(0.5 \mathrm{mmol})$ as a white solid $(175 \mathrm{mg}, 91 \%)$. $[\alpha]_{\mathrm{D}}^{26}=-226.0\left(c\right.$ 1.0, $\left.\mathrm{CHCl}_{3}\right)$; M.pt. $196-198{ }^{\circ} \mathrm{C} ;{ }^{1} \mathrm{H} \mathrm{NMR}$ $\left(400 \mathrm{MHz}, \mathrm{CDCl}_{3}\right): \delta 7.22-7.03(\mathrm{~m}, 8 \mathrm{H}), 6.88(\mathrm{~d}, J=7.2 \mathrm{~Hz}, 2$ $\mathrm{H}), 5.79(\mathrm{~d}, J=3.6 \mathrm{~Hz}, 1 \mathrm{H}), 4.89(\mathrm{dd}, J=2.8,8.8 \mathrm{~Hz}, 1 \mathrm{H})$, 4.27 (m, 2H), 3.83-3.66 (m, 2H), 3.57-3.48 (m, 1H), 3.42 (d, $J$ $=7.6 \mathrm{~Hz}, 1 \mathrm{H}), 3.56(\mathrm{~d}, J=12.8 \mathrm{~Hz}, 1 \mathrm{H}), 2.20-2.19(\mathrm{~m}, 3 \mathrm{H})$, $1.06(\mathrm{t}, J=7.2 \mathrm{~Hz}, 3 \mathrm{H}) \mathrm{ppm} ;{ }^{13} \mathrm{C}\left\{{ }^{1} \mathrm{H}\right\} \mathrm{NMR}(100 \mathrm{MHz}$, $\left.\mathrm{CDCl}_{3}\right): \delta 174.6,170.8,135.4,134.2,129.5,128.1,128.0$, 128.0, 128.0, 127.8, 126.7, 83.5, 77.3, 77.0, 76.6, 64.6, 64.2, 61.1, 57.4, 50.1, 35.4, 31.7, 13.6, 13.6, $13.6 \mathrm{ppm}$; $v_{\max }($ neat $) / \mathrm{cm}^{-1}: 3481,2932,1699,1232,1052,697 \mathrm{~cm}^{-1}$; HRMS (ESI) $m / z$ : [M] $]^{+}$calcd for $\mathrm{C}_{23} \mathrm{H}_{26} \mathrm{O}_{5} \mathrm{~N} 396.1805$; found 396.1806 .

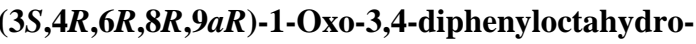
pyrido $[2,1-c][1,4]$ oxazine-6,8-carboxylic anhydride $(9 \mathrm{~h}$, Table 2 entry 9). Following the general procedure, $\mathbf{A}$, the title compound was prepared prepared from compound7a (146 mg) and glyoxylic acid as a white solid (104 mg, $60 \%)$. $[\alpha]_{\mathrm{D}}{ }^{26}=$ +40.0 (c 1.0, MeOH); M.pt. $240-242{ }^{\circ} \mathrm{C} ;{ }^{1} \mathrm{H} \mathrm{NMR}(400 \mathrm{MHz}$, $\left.\mathrm{CDCl}_{3}\right): \delta$ 7.14-7.05 (m, $\left.6 \mathrm{H}\right), 6.95-6.88(\mathrm{~m}, 4 \mathrm{H}), 5.72(\mathrm{~d}, J=$ $5.6 \mathrm{~Hz}, 1 \mathrm{H}), 5.00(\mathrm{t}, J=5.6 \mathrm{~Hz}, 1 \mathrm{H}), 4.47(\mathrm{~d}, J=4.8 \mathrm{~Hz}, 1$ $\mathrm{H}), 4.12(\mathrm{dd}, J=5.6 \mathrm{~Hz}, 1 \mathrm{H}), 3.35(\mathrm{~d}, J=4.8 \mathrm{~Hz}, 1 \mathrm{H}), 2.62-$ $2.54(\mathrm{~m}, 1 \mathrm{H}), 2.30-2.18(\mathrm{~m}, 2 \mathrm{H}), 1.93(\mathrm{~m}, 1 \mathrm{H}) \mathrm{ppm} ;{ }^{13} \mathrm{C}\left\{{ }^{1} \mathrm{H}\right\}$ NMR (100 MHz, $\left.\mathrm{CDCl}_{3}\right)$ : $\delta 173.4,170.6,136.1,134.2,129.1$, 128.2, 128.2, 127.9, 127.9, 126.7, 126., 79.6, 77.4, 77.1, 76.7, $76.5,65.8,57.8,52.8,36.9,31.2 \mathrm{ppm} ; v_{\max }($ neat $) / \mathrm{cm}^{-1}: 2825$, 1756, 1218, 1049, 956, 771, $678 \mathrm{~cm}^{-1}$; HRMS (ESI) $\mathrm{m} / \mathrm{z}:[\mathrm{M}]^{+}$ calcd for $\mathrm{C}_{21} \mathrm{H}_{20} \mathrm{O}_{4} \mathrm{~N} 350.1387$; found 350.1390.

General procedure-B for the aza-Prins- Ritter reaction: A round-bottomed flask was charged with an aldehyde (0.5 eq.) and $\mathrm{CH}_{3} \mathrm{CN}(10 \mathrm{~mL})$. To the resulting suspension was added anhydrous pTSA ( $0.75 \mathrm{mmol}, 1.5$ eq. $)$. After stirring the mixture for $15 \mathrm{~min}$ at room temperature, an amine derivative (7a or $7 \mathbf{b}, 0.5 \mathrm{mmol}, 1.00$ eq.) in $\mathrm{CH}_{3} \mathrm{CN}(3 \mathrm{~mL})$ was added and the resulting mixture stirred for $12 \mathrm{hrs}$ at $35^{\circ} \mathrm{C}$ using a solid heating block. The solvent was removed and diluted with DCM $(20 \mathrm{~mL})$ followed by sat. $\mathrm{NaHCO}_{3}$ solution $(20 \mathrm{~mL})$. The organic layer was separated and the aqueous layer was extracted with dichloromethane $(2 \times 10 \mathrm{~mL})$. The combined organic layers were dried over magnesium sulfate, filtered and concentrated in vacuo, and purified by chromatography (silica gel 100-200 mesh, 50:49:1 EtOAc/Hexane/Et 3 N). 
$N$-((3S,4R,6S,8R,9aR)-1-oxo-6-phenethyl-3,4-diphenyloctahydropyrido[2,1-c] [1,4] oxazin-8-yl) acetamide (10a, Table 3 Entry 1). Following the general procedure B, the title compound 10a was prepared from compound 7a (146 $\mathrm{mg}$ ) and 3-phenylpropionaldehyde as a white solid (194 mg, $83 \%) .[\alpha]_{\mathrm{D}}^{26}=-560.00$ (c 1.0, CHCl3); M.pt. $166-168^{\circ} \mathrm{C} ;{ }^{1} \mathrm{H}$ NMR (400 MHz, $\left.\mathrm{CDCl}_{3}\right): \delta$ 7.22-7.02 (m, $\left.10 \mathrm{H}\right), 6.97-6.92$ $(\mathrm{m}, 3 \mathrm{H}), 6.74-6.70(\mathrm{~m}, 2 \mathrm{H}), 5.51(\mathrm{~d}, J=4.0 \mathrm{~Hz}, 1 \mathrm{H}), 5.37$ (d, $J=8.3 \mathrm{~Hz}, 1 \mathrm{H}), 4.53(\mathrm{~d}, J=4.0 \mathrm{~Hz}, 1 \mathrm{H}), 4.38-4.18(\mathrm{~m}, 2$ H), 3.05-2.97 (m, $1 \mathrm{H}), 2.67-2.57(\mathrm{~m}, 1 \mathrm{H}), 2.46-2.32(\mathrm{~m}, 1$ H), $1.96(\mathrm{~s}, 3 \mathrm{H}), 1.90-1.78(\mathrm{~m}, 1 \mathrm{H}), 1.70-1.55(\mathrm{~m}, 3 \mathrm{H})$, $1.71 .37(\mathrm{~m}, 1 \mathrm{H}) \mathrm{ppm} ;{ }^{13} \mathrm{C}\left\{{ }^{1} \mathrm{H}\right\}$ NMR $\left(100 \mathrm{MHz}, \mathrm{CDCl}_{3}\right): \delta$ $170.5,169.3,141.6,141.5,135.5,135.4,129.8,128.4,128.4$, $128.3,128.3,128.3,128.1,127.9,127.7,125.9,84.1,77.4$, 77.3, 77.1, 76.8, 62.6, 54.4, 42.9, 32.8, 32.4, 31.8, 31.2, 23.6 ppm; $v_{\max }($ neat $) / \mathrm{cm}^{-1}: 2980,1738,1544,1373,1235,1020$, $694 \mathrm{~cm}^{-1}$; HRMS (ESI) $\mathrm{m} / \mathrm{z}:[\mathrm{M}]^{+}$calcd for $\mathrm{C}_{30} \mathrm{H}_{33} \mathrm{O}_{3} \mathrm{~N}_{2}$ 469.2486; found 469.2481.

$N$-((3R,4S,6R,8S,9aS)-1-Oxo-6-phenethyl-3,4-diphenyloctahydropyrido[2,1-c] [1,4] oxazin-8-yl) acetamide (10a', Table 3 Entry 2). Following the general procedure B, the title compound 10a' (enantiomer of 10a) was prepared from compound $7 \mathbf{b}(146 \mathrm{mg})$ and 3-phenylpropionaldehyde as a white solid $(178 \mathrm{mg}, 76 \%)$. $[\alpha]_{\mathrm{D}}^{26}=+120.00(\mathrm{c} 1.0, \mathrm{MeOH})$; M.pt. $172-174{ }^{\circ} \mathrm{C} ;{ }^{1} \mathrm{H}$ NMR $\left(400 \mathrm{MHz}, \mathrm{CDCl}_{3}\right): \delta$ 7.22-7.02 $(\mathrm{m}, 10 \mathrm{H}), 6.97-6.92(\mathrm{~m}, 3 \mathrm{H}), 6.74-6.70(\mathrm{~m}, 2 \mathrm{H}), 5.51(\mathrm{~d}, J=$ $4.0 \mathrm{~Hz}, 1 \mathrm{H}), 5.37(\mathrm{~d}, J=8.3 \mathrm{~Hz}, 1 \mathrm{H}), 4.53(\mathrm{~d}, J=4.0 \mathrm{~Hz}, 1$ H), 4.38-4.18 (m, 2 H), 3.05-2.97 (m, $1 \mathrm{H}), 2.67-2.57$ (m, 1 H), 2.46-2.32 (m, $1 \mathrm{H}), 1.96(\mathrm{~s}, 3 \mathrm{H}), 1.90-1.78(\mathrm{~m}, 1 \mathrm{H}), 1.70-$ $1.55(\mathrm{~m}, 3 \mathrm{H}), 1.71-1.37(\mathrm{~m}, 1 \mathrm{H}) \mathrm{ppm} ;{ }^{13} \mathrm{C}\left\{{ }^{1} \mathrm{H}\right\}$ NMR $(100$ $\left.\mathrm{MHz}, \mathrm{CDCl}_{3}\right): \delta 169.4,168.3,140.5,134.5,134.4,128.7$, 127.4, 127.4, 127.3, 127.2, 127.1, 126.9, 126.6, 124.9, 83.0, 76.3, 76.1, 75.8, 61.6, 53.4, 53.3, 41.9, 31.8, 31.4, 30.7, 30.2, $22.6 \mathrm{ppm} ; \nu_{\max }($ neat $) / \mathrm{cm}^{-1}: 2981,1733,1355,1114,751,698$ $\mathrm{cm}^{-1}$; HRMS (ESI) $\mathrm{m} / z$ : [M] $]^{+}$calcd for $\mathrm{C}_{30} \mathrm{H}_{33} \mathrm{O}_{3} \mathrm{~N}_{2}$ 469.2486; found 469.2481.

$N$-((3S,4R,6R,8S,9aR)-6-Heptyl-1-oxo-3,4-diphenyloctahydropyrido[2,1-c] [1,4] oxazin-8-yl) acetamide (10b, Table 3 Entry 3). Following the general procedure $\mathbf{B}$, the title compound was prepared from compound $7 \mathbf{a}(146 \mathrm{mg})$ and octanal as a white solid $(168 \mathrm{mg}, 73 \%) .[\alpha]_{\mathrm{D}}{ }^{26}=-54.0\left(c 1.0, \mathrm{CHCl}_{3}\right)$; M.pt. $172-174{ }^{\circ} \mathrm{C} ;{ }^{1} \mathrm{H}$ NMR $\left(400 \mathrm{MHz}, \mathrm{CDCl}_{3}\right): \delta$ 7.23-7.06 $(\mathrm{m}, 6 \mathrm{H}), 6.94-6.91(\mathrm{~m}, 2 \mathrm{H}), 6.77-6.72(\mathrm{~m}, 2 \mathrm{H}), 5.49(\mathrm{~d}, J=$ $4.0 \mathrm{~Hz}, 1 \mathrm{H}), 5.32$ (bs, $1 \mathrm{H}), 4.54$ (d, $J=4.0 \mathrm{~Hz}, 1 \mathrm{H}), 4.31-$ $4.16(\mathrm{~m}, 2 \mathrm{H}), 2.96-2.92(\mathrm{~m}, 1 \mathrm{H}), 2.36-2.31(\mathrm{~m}, 1 \mathrm{H}), 1.96$ (s, $3 \mathrm{H}), 1.81-1.77(\mathrm{~m}, 1 \mathrm{H}), 1.55-1.49(\mathrm{~m}, 2 \mathrm{H}), 1.40-1.08(\mathrm{~m}, 11$ $\mathrm{H}), 0.86$ (t, $J=7.2 \mathrm{~Hz}, 3 \mathrm{H}) \mathrm{ppm} ;{ }^{13} \mathrm{C}\left\{{ }^{1} \mathrm{H}\right\} \mathrm{NMR}(100 \mathrm{MHz}$, $\left.\mathrm{CDCl}_{3}\right): \delta 170.5,169.2,135.6,135.5,129.8,128.3,128.2$, 128.1, 127.9, 127.6, 84.2, 77.4, 77.1, 76.7, 62.3, 54.6, 54.3, 42.9, 32.2, 31.8, 31.0, 30.3, 29.5, 29.3, 26.7, 23.6, 22.7, 14.2 ppm; $v_{\max }($ neat $) / \mathrm{cm}^{-1}: 2981,1733,1373,1132,702 \mathrm{~cm}^{-1}$; HRMS (ESI) $m / z$ : [M] $]^{+}$calcd for $\mathrm{C}_{29} \mathrm{H}_{39} \mathrm{O}_{3} \mathrm{~N}_{2} 463.2955$; found 463.2951 .

$N$-((3S,4R,6S,8R,9aR)-6-nonyl-1-oxo-3,4-diphenyloctahydropyrido[2,1-c] [1,4] oxazin-8-yl) acetamide (10c, Table 3 Entry 4). Following the general procedure $\mathbf{B}$, the title compound 10c was prepared from compound $7 \mathbf{a}(146 \mathrm{mg})$ and decanal as a white solid $(191 \mathrm{mg}, 78 \%)$. $[\alpha]_{\mathrm{D}}{ }^{26}=-132.0(c$ 1.0, $\mathrm{CHCl}_{3}$ ); M.pt. $173-175{ }^{\circ} \mathrm{C} ;{ }^{1} \mathrm{H} \mathrm{NMR}\left(400 \mathrm{MHz}, \mathrm{CDCl}_{3}\right): \delta$ 7.22-7.03 (m, $6 \mathrm{H}), 6.95-6.90$ (m, $2 \mathrm{H}), 6.78-6.72$ (m, $2 \mathrm{H})$, $5.47(\mathrm{~d}, J=4.0 \mathrm{~Hz}, 1 \mathrm{H}), 5.38(\mathrm{~d}, J=8.4 \mathrm{~Hz}, 1 \mathrm{H}), 4.54(\mathrm{~d}, J=$ $4.0 \mathrm{~Hz}, 1 \mathrm{H}), 4.28-4.18$ (m, $2 \mathrm{H}), 2.98-2.93$ (m, $1 \mathrm{H}), 2.38-$ $2.30(\mathrm{~m}, 1 \mathrm{H}), 1.97$ (s, 3H), 1.83-1.77 (m, $1 \mathrm{H}), 1.57-1.49(\mathrm{~m}$,
$1 \mathrm{H}), 1.40-1.02(\mathrm{~m}, 16 \mathrm{H}), 0.87$ (d, $J=7.2 \mathrm{~Hz}, 3 \mathrm{H}) \mathrm{ppm}$; ${ }^{13} \mathrm{C}\left\{{ }^{1} \mathrm{H}\right\}$ NMR $\left(100 \mathrm{MHz}, \mathrm{CDCl}_{3}\right): \delta 170.5,169.2,135.6$, $135.5,129.8,128.3,128.2,128.1,127.9,127.6,84.2,77.4$, $77.1,76.7,62.3,54.6,54.3,42.9,32.2,31.9,31.0,30.3,29.6$, 29.4, 26.7, 23.6, 22.7, $14.2 \mathrm{ppm} ; v_{\max }$ (neat) $/ \mathrm{cm}^{-1}: 2971,1733$, 1319, 1053, 954, $768 \mathrm{~cm}^{-1}$; HRMS (ESI) $\mathrm{m} / \mathrm{z}:[\mathrm{M}]^{+}$calcd for $\mathrm{C}_{31} \mathrm{H}_{43} \mathrm{O}_{3} \mathrm{~N}_{2}$ 491.3268; found 491.3259.

$N$-((3S,4R,6S,8R,9aR)-1-Oxo-6-pentyl-3,4-diphenyloctahydropyrido[2,1-c] $[1,4]$ oxazin-8-yl) acetamide (10d, Table 3 Entry 5). Following the general procedure B, the title compound 10d was prepared from compound $7 \mathbf{a}(146 \mathrm{mg})$ and hexanal as a white solid $(163 \mathrm{mg}, 75 \%)$. $[\alpha]_{\mathrm{D}}^{26}=-120.0(c$ 1.0, $\left.\mathrm{CHCl}_{3}\right)$; M.pt. $156-158{ }^{\circ} \mathrm{C} ;{ }^{1} \mathrm{H}$ NMR (400 MHz, $\left.\mathrm{CDCl}_{3}\right): \delta$ 7.22-7.06 (m, $6 \mathrm{H}), 6.96-6.91$ (m, $2 \mathrm{H}), 6.78-6.72(\mathrm{~m}, 2 \mathrm{H})$, 5.49 (d, $J=4.0 \mathrm{~Hz}, 1 \mathrm{H}), 5.32$ (bs, $1 \mathrm{H}), 4.55$ (d, $J=4.0 \mathrm{~Hz}, 1$ $\mathrm{H}), 4.31-4.16$ (m, $2 \mathrm{H}), 2.98-2.92$ (m, $1 \mathrm{H}), 2.38-2.29$ (m, 1 $\mathrm{H}), 1.96$ (s, 3H), 1.87-1.83 (m, $1 \mathrm{H}), 1.55-1.49$ (m, $2 \mathrm{H}), 0.81$ $(\mathrm{t}, J=7.2 \mathrm{~Hz}, 3 \mathrm{H}) \mathrm{ppm} ;{ }^{13} \mathrm{C}\left\{{ }^{1} \mathrm{H}\right\} \mathrm{NMR}\left(100 \mathrm{MHz}, \mathrm{CDCl}_{3}\right): \delta$ 170.5, 169.2, 135.6, 135.4, 129.8, 128.3, 128.2, 128.1, 128.0, 128.0, 127.9, 127.6, 84.2, 77.4, 77.3, 77.1, 76.7, 62.3, 54.6, $54.3,42.9,32.2,31.7,31.0,30.2,26.4,23.6,22.6,22.6,14.0$ ppm; $v_{\max }($ neat $) / \mathrm{cm}^{-1}: 2981,1717,1237,1042,753,700 \mathrm{~cm}^{-1}$; HRMS (ESI) $m / z$ : [M] $]^{+}$calcd for $\mathrm{C}_{27} \mathrm{H}_{35} \mathrm{O}_{3} \mathrm{~N}_{2}$ 435.2642; found 435.2641.

$N$-((3S,4R,6S,8R,9aR)-1-oxo-3,4-diphenyl-6-propyloctahydropyrido[2,1-c] $[1,4]$ oxazin-8-yl) acetamide (10e, Table 3 Entry 6). Following the general procedure B, the title compound 10e was prepared from compound $7 \mathbf{a}(146 \mathrm{mg})$ and butanal as a white solid $(152 \mathrm{mg}, 74 \%) .[\alpha]_{\mathrm{D}}{ }^{26}=-63.0(c 1.0$, $\mathrm{CHCl}_{3}$ ); M.pt. $162-164{ }^{\circ} \mathrm{C} ;{ }^{1} \mathrm{H}$ NMR (400 MHz, $\left.\mathrm{CDCl}_{3}\right): \delta$ 7.22-7.03 (m, $6 \mathrm{H}), 6.95-6.90$ (m, $2 \mathrm{H}), 6.78-6.72(\mathrm{~m}, 2 \mathrm{H})$, 5.49 (d, $J=4.0 \mathrm{~Hz}, 1 \mathrm{H}), 5.32$ (bs, $1 \mathrm{H}), 4.55(\mathrm{~d}, J=4.0 \mathrm{~Hz}, 1$ H), 4.31-4.16 (m, $2 \mathrm{H}), 2.98-2.92(\mathrm{~m}, 1 \mathrm{H}), 2.38-2.20$ (m, 1 $\mathrm{H}), 1.96$ (s, 3H), 1.81-1.77 (m, $1 \mathrm{H}), 1.40-1.22$ (m, $4 \mathrm{H}), 0.75$ $(\mathrm{t}, J=7.2 \mathrm{~Hz}, 3 \mathrm{H}) \mathrm{ppm} ;{ }^{13} \mathrm{C}\left\{{ }^{1} \mathrm{H}\right\} \mathrm{NMR}\left(100 \mathrm{MHz}, \mathrm{CDCl}_{3}\right): \delta$ 170.5, 169.2, 135.6, 135.5, 129.8, 128.3, 128.2, 128.0, 128.0, 127.6, 84.1, 77.4, 77.2, 77.1, 76.7, 62.3, 54.5, 54.3, 42.9, 32.6, $32.2,30.9,23.6,19.9,14.1 \mathrm{ppm} ; v_{\max }$ (neat) $/ \mathrm{cm}^{-1}: 2981,1732$, 1232, 1035, 912, $694 \mathrm{~cm}^{-1}$; HRMS (ESI) $\mathrm{m} / z$ : [M]+ calcd for $\mathrm{C}_{25} \mathrm{H}_{31} \mathrm{O}_{3} \mathrm{~N}_{2}$ : 407.2329; found: 407.2325. Compound 10e was also prepared using the same method starting from $7 \mathbf{a}$ (146 mg) and butanal diethyl acetal (73 mg) in $131 \mathrm{mg}, 65 \%$ yield (Table 3 Entry 7). All data was in agreement with that presented above.

$N$-((3S,4R,6S,8R,9aR)-6-benzyl-1-oxo-3,4-diphenyloctahydropyrido[2,1-c] $[1,4]$ oxazin-8-yl) acetamide (10f, Table 3 Entry 8). Following the general procedure B, the title compound 10f was prepared from compound 7a (146 mg) and phenylacetaldehyde as a white solid $(186 \mathrm{mg}, 82 \%) .[\alpha]_{\mathrm{D}}{ }^{26}=-$ 200.00 (c 1.0, MeOH); M.pt. 156-158 ${ }^{\circ} \mathrm{C} ;{ }^{1} \mathrm{H}$ NMR (400 MHz, $\left.\mathrm{CDCl}_{3}\right): \delta$ 7.22-6.99 (m, $\left.12 \mathrm{H}\right), 6.84-6.79(\mathrm{~m}, 2 \mathrm{H}), 6.63-6.53$ $(\mathrm{m}, 1 \mathrm{H}), 5.47(\mathrm{~d}, J=4.0 \mathrm{~Hz}, 1 \mathrm{H}), 5.35(\mathrm{bs}, 1 \mathrm{H}), 4.53(\mathrm{~d}, J=$ $4.0 \mathrm{~Hz}, 1 \mathrm{H}), 4.46-4.34$ (m, $1 \mathrm{H}), 3.31-3.21$ (m, $1 \mathrm{H}), 2.72-$ $2.66(\mathrm{~m}, 1 \mathrm{H}), 2.52-2.44(\mathrm{~m}, 1 \mathrm{H}), 1.95(\mathrm{~s}, 3 \mathrm{H}), 1.88-1.77(\mathrm{~m}, 1$ $\mathrm{H}), 1.38-1.28(\mathrm{~m}, 1 \mathrm{H}) \mathrm{ppm} ;{ }^{13} \mathrm{C}\left\{{ }^{1} \mathrm{H}\right\} \mathrm{NMR}(100 \mathrm{MHz}$, $\left.\mathrm{CDCl}_{3}\right): \delta 170.1,169.2,139.1,135.4,135.3,129.7,129.3$, $128.4,128.2$, 128.1, 128.1, 127.8, 127.7, 126.3, 84.5, 77.4, 77.3, 77.1, 76.8, 62.7, 57.4, 54.4, 42.9, 36.3, 32.9, 30.5, 23.5 ppm; $v_{\max }($ neat $) / \mathrm{cm}^{-1}: 2981,1734,1653,1454,1381,1239$, 1071, $698 \mathrm{~cm}^{-1}$; HRMS (ESI) $\mathrm{m} / \mathrm{z}$ : [M] ${ }^{+}$calcd for $\mathrm{C}_{29} \mathrm{H}_{31} \mathrm{O}_{3}$ $\mathrm{N}_{2}$ 455.2329; found 455.2323 . Compound 10f was also prepared using the same method starting from $7 \mathbf{a}(146 \mathrm{mg})$ and 
styrene oxide (60 mg) in $142 \mathrm{mg}$, 63\% yield (Table 3 Entry 9). All data was in agreement with that presented above.

$N$-((3S,4R,6R,8R,9aR)-6-benzhydryl-1-oxo-3,4-diphenyloctahydropyrido[2,1-c] $[1,4]$ oxazin-8-yl) acetamide (10g, Table 3 Entry 10). Following the general procedure B, the title compound $\mathbf{1 0 g}$ was prepared from compound $7 \mathbf{a}$ (146 $\mathrm{mg}$ ) and diphenyl acetaldehyde as a white solid (207 mg, 78 $\%$ ). $[\alpha]_{\mathrm{D}}{ }^{26}=-113.0$ (c 1.0, $\left.\mathrm{CHCl}_{3}\right)$; M.pt. $148-150{ }^{\circ} \mathrm{C} ;{ }^{1} \mathrm{H}$ NMR $\left(400 \mathrm{MHz}, \mathrm{CDCl}_{3}\right): \delta$ 7.32-7.02 $(\mathrm{m}, 15 \mathrm{H}), 6.88(\mathrm{t}, J=$ 7.6 Hz, $2 \mathrm{H}), 6.59-6.45$ (m, $3 \mathrm{H}), 5.30-5.19$ (m, $2 \mathrm{H}), 4.67$ (d, $J=4.1 \mathrm{~Hz}, 1 \mathrm{H}), 4.51-4.46(\mathrm{~m}, 1 \mathrm{H}), 4.33(\mathrm{~d}, J=12.8 \mathrm{~Hz}, 1$ $\mathrm{H}), 3.74-3.61(\mathrm{~m}, 1 \mathrm{H}), 2.52-2.43(\mathrm{~m}, 1 \mathrm{H}), 2.05-1.94(\mathrm{~m}, 1 \mathrm{H})$, $1.89(\mathrm{~s}, 3 \mathrm{H}) \mathrm{ppm} ;{ }^{13} \mathrm{C}\left\{{ }^{1} \mathrm{H}\right\} \mathrm{NMR}\left(100 \mathrm{MHz}, \mathrm{CDCl}_{3}\right): \delta 169.6$, 169.3, 142.3, 141.8, 135.3, 134.3, 130.4, 128.9, 128.6, 128.38, 128.0, 127.9, 127.9, 127.8, 127.7, 126.9, 126.8, 84.1, 77.4, 77.1, 76.7, 61.9, 57.7, 54.8, 52.2, 42.2, 30.8, 26.4, 23.5 ppm; $v_{\max }($ neat $) / \mathrm{cm}^{-1}: 2982,1796,1412,1096,978,693 \mathrm{~cm}^{-1}$; HRMS (ESI) $m / z$ : [M] $]^{+}$calcd for $\mathrm{C}_{35} \mathrm{H}_{35} \mathrm{O}_{3} \mathrm{~N}_{2}$ 531.2642; found 531.2637.

General procedure $\mathrm{C}$ : for the $\mathrm{FeCl}_{3}$ or $\mathrm{FeBr}_{3}$ catalysed aza-Prins reaction: A round-bottomed flask was charged with aldehyde (0.5 eq.) and solvent (DCM for $\mathrm{FeCl}_{3}$ and $\mathrm{DBE}$ for $\left.\mathrm{FeBr}_{3}\right)(10 \mathrm{~mL})$. To the resulting suspension was added $\mathrm{FeCl}_{3}$ or $\mathrm{FeBr}_{3}(0.75 \mathrm{mmol}, 1.5$ eq. $)$ at $0{ }^{\circ} \mathrm{C}$. After stirring the mixture for $15 \mathrm{~min}$ at same temperature, the corresponding amine derivative $(0.5 \mathrm{mmol}, 1.00$ eq. $)$ in the appropriate solvent $(\mathrm{DCM} / \mathrm{DBE})(3 \mathrm{~mL})$ was added and the resulting mixture stirred for $6 \mathrm{hrs}$. After completion of the reaction, resulting solution treated with aqueous sodium bicarbonate $(5 \mathrm{~mL})$ and the product was extracted with dichloromethane $(2 \times 10 \mathrm{~mL})$. The organic layer was washed with brine $(5 \mathrm{~mL})$, dried over $\mathrm{MgSO}_{4}$ and evaporated to leave the crude product, which was purified by column chromatography (silica gel 100-200 mesh; 5: 95 EtOAc/Hexane).

General procedure D: for the O-tosyl azabicyclic derivatives via aza-Prins reaction: A round-bottomed flask was charged with aldehyde $\left(0.5\right.$ eq.) and $\mathrm{CH}_{3} \mathrm{CN}(10 \mathrm{~mL})$. To the resulting suspension was added anhydrous PTSA $(0.75 \mathrm{mmol}$, 1.5 eq.). After stirring the mixture for $15 \mathrm{~min}$ at room temperature, the corresponding amine derivative $(0.5 \mathrm{mmol}, 1.00$ eq. $)$ in $\mathrm{CH}_{3} \mathrm{CN}$ ( $3 \mathrm{~mL}$ ) was added and the resulting mixture stirred for $12 \mathrm{hrs}$. After completion of the reaction, the solvent was concentrated. The resulting crude compound was treated with aqueous sodium bicarbonate $(5 \mathrm{~mL})$ and the product was extracted with dichloromethane $(2 \times 10 \mathrm{~mL})$. The organic layer was washed with brine $(5 \mathrm{~mL})$, dried over $\mathrm{MgSO}_{4}$ and evaporated to leave the crude product, which was purified by column chromatography (silica gel 100-200 mesh; 5: 95 EtOAc/Hexane).

General procedure E: for the aza-Prins-Ritter reaction: a) Using $\mathrm{BF}_{3}$. $\mathrm{OEt}_{2}$ : a round-bottomed flask was charged with aldehyde (0.5 eq.) and $\mathrm{CH}_{3} \mathrm{CN}(10 \mathrm{~mL})$. To the resulting suspension was added $\mathrm{BF}_{3} . \mathrm{OEt}_{2}(0.75 \mathrm{mmol}, 1.5$ eq. $)$. After stirring the mixture for $15 \mathrm{~min}$ at room temperature, the corresponding amine derivative ( $0.5 \mathrm{mmol}, 1.00$ eq.) in $\mathrm{CH}_{3} \mathrm{CN}$ ( $3 \mathrm{~mL}$ ) was added and the resulting mixture stirred for $12 \mathrm{hrs}$. After completion of the reaction, the solvent was concentrated. The resulting crude compound was treated with aqueous sodium bicarbonate $(5 \mathrm{~mL})$ and the product was extracted with dichloromethane $(2 \times 10 \mathrm{~mL})$. The organic layer was washed with brine $\left(5 \mathrm{~mL}\right.$ ), dried over $\mathrm{MgSO}_{4}$ and evaporated to leave the crude product, which was purified by column chromatography (silica gel 100-200 mesh; 50: 49: 1 EtOAc/Hexane/Et 3 N). b) Using $\mathrm{Sc}(\mathrm{OTf})_{3}$ : a round-bottomed flask was charged with aldehyde (0.5 eq.) and DCM $(10 \mathrm{~mL})$. To the resulting suspension was added $\mathrm{Sc}(\mathrm{OTf})_{3}(0.75 \mathrm{mmol}, 1.5 \mathrm{eq}$. $)$. After stirring the mixture for $15 \mathrm{~min}$ at room temperature, the corresponding amine derivative (3, $0.5 \mathrm{mmol}, 1.00$ eq.) in DCM (3 mL) was added and the resulting mixture stirred for $12 \mathrm{hrs}$. After completion of the reaction, the solvent was concentrated. The resulting crude compound was treated with aqueous sodium bicarbonate $(5 \mathrm{~mL})$ and the product was extracted with dichloromethane $(2 \times 10 \mathrm{~mL})$. The organic layer was washed with brine $(5 \mathrm{~mL})$, dried over $\mathrm{MgSO}_{4}$ and evaporated to leave the crude product, which was purified by column chromatography (silica gel 100-200 mesh; 50: 49: 1 EtOAc/Hexane/Et $3 \mathrm{~N}$ ).

(4S,6R,8R,9aS)-8-chloro-6-phenethyl-4-phenylhexahydropyrido[2,1-c] [1,4] oxazin-1(6H)-one (12a, Table 4 Entry 2). Following the general procedure $\mathbf{C}$ the title compound $\mathbf{1 2 a}$ was prepared from compound $\mathbf{8 a}(109 \mathrm{mg})$ and 3-phenylpropionaldehyde as a white solid $(166 \mathrm{mg}, 90 \%) .[\alpha]_{\mathrm{D}}{ }^{26}=$ +143.0 (c 1.0, $\mathrm{CHCl}_{3}$ ); M.pt. 132-134 ${ }^{\circ} \mathrm{C}$; ${ }^{1} \mathrm{H}$ NMR (400 MHz, $\left.\mathrm{CDCl}_{3}\right): \delta$ 7.37-7.14 (m, $\left.8 \mathrm{H}\right), 7.11-7.08(\mathrm{~m}, 2 \mathrm{H}), 4.37-4.17$ $(\mathrm{m}, 4 \mathrm{H}), 4.11(\mathrm{dd}, J=3.2,9.2 \mathrm{~Hz}, 1 \mathrm{H}), 2.89-2.70(\mathrm{~m}, 2 \mathrm{H})$, 2.45-2.20 (m, $3 \mathrm{H}), 1.95-1.65$ (m, 3H), 1.61-1.54 (m, $1 \mathrm{H})$ ppm; ${ }^{13} \mathrm{C}\left\{{ }^{1} \mathrm{H}\right\}$ NMR $\left(100 \mathrm{MHz}, \mathrm{CDCl}_{3}\right): \delta 169.2,141.5$, 135.4, 129.4, 129.2, 128.6, 128.6, 128.2, 126.1, 77.4, 77.1, 76.7, 73.9, 55.8, 55.6, 54.2, 53.6, 34.9, 33.9, 33.1, 32.8 ppm; $v_{\max }($ neat $) / \mathrm{cm}^{-1}: 2981,1726,1249,1133,1020,698 \mathrm{~cm}^{-1}$; HRMS (ESI) $m / z:[\mathrm{M}]^{+}$calcd for $\mathrm{C}_{22} \mathrm{H}_{25}{ }^{35} \mathrm{ClNO}_{2} 370.1574$; found 370.1570; calcd for $\mathrm{C}_{22} \mathrm{H}_{25}{ }^{37} \mathrm{ClNO}_{2} 372.1544$; found 372.1536 .

(4S,6S,8R,9aS)-8-Chloro-4,6-diphenylhexahydropyrido[2,1-c] $[1,4]$ oxazin-1 $(6 H)$-one $(12 b$, Table 4 Entry 3). Following the general procedure $\mathbf{C}$, the title compound $\mathbf{1 2 b}$ was prepared from compound $\mathbf{8 a}(109 \mathrm{mg})$ and benzaldehyde as a white solid $(155 \mathrm{mg}, 91 \%)$. $[\alpha]_{\mathrm{D}}{ }^{26}=+78.0\left(c 1.0, \mathrm{CHCl}_{3}\right)$; M.pt. $164-166{ }^{\circ} \mathrm{C} ;{ }^{1} \mathrm{H}$ NMR $\left(400 \mathrm{MHz}, \mathrm{CDCl}_{3}\right): \delta 7.56-7.23$ $(\mathrm{m}, 10 \mathrm{H}), 4.59-4.51(\mathrm{~m}, 2 \mathrm{H}), 4.38-4.36(\mathrm{~m}, 1 \mathrm{H}), 4.25-4.16$ (m, $2 \mathrm{H}), 3.86$ (dd, $J=4.0 \mathrm{~Hz}, 1 \mathrm{H}), 2.55-2.39$ (m, $2 \mathrm{H}), 2.28-$ 2.21 (m, $1 \mathrm{H}), 2.10-2.03(\mathrm{~m}, 1 \mathrm{H}) \mathrm{ppm} ;{ }^{13} \mathrm{C}\left\{{ }^{1} \mathrm{H}\right\}$ NMR $(100$ $\left.\mathrm{MHz}, \mathrm{CDCl}_{3}\right): \delta 169.3,139.3,136.0,129.4,129.1,129.0$, $128.0,127.5,126.7,71.5,56.2,55.2,54.6,53.6,34.4,33.8$ ppm; $v_{\max }($ neat $) / \mathrm{cm}^{-1}: 3031,1783,1202,1163,1104,784 \mathrm{~cm}^{-1}$; HRMS (ESI) $m / z$ : [M] ${ }^{+}$calcd for $\mathrm{C}_{20} \mathrm{H}_{21}{ }^{35} \mathrm{ClNO}_{2} 342.1261$; found 342.1256; calcd for $\mathrm{C}_{20} \mathrm{H}_{21}{ }^{37} \mathrm{ClNO}_{2} 344.1231$; found 344.1234 .

(4S,6S,8R,9aS)-6-Benzhydryl-8-chloro-4-phenylhexahydropyrido[2,1-c] [1,4] oxazin-1(6H)-one (12c, Table 4 Entry 4). Following the general procedure $\mathbf{C}$, the title compound was prepared from compound $\mathbf{8 a}(109 \mathrm{mg})$ and diphenyl acetaldehyde as a white solid $(192 \mathrm{mg}, 89 \%)$. $[\alpha]_{\mathrm{D}}{ }^{26}=+132.0(c$ 1.0, $\mathrm{CHCl}_{3}$ ); M.pt. $120-122{ }^{\circ} \mathrm{C} ;{ }^{1} \mathrm{H}$ NMR (400 MHz, $\left.\mathrm{CDCl}_{3}\right): \delta$ 7.43-7.32 (m, $3 \mathrm{H}), 7.26-7.13(\mathrm{~m}, 6 \mathrm{H}), 7.09-7.00$ (m, $4 \mathrm{H})$, 6.92-6.86 (m, $2 \mathrm{H}), 4.37-4.19$ (m, $3 \mathrm{H}), 4.13-3.97$ (m, $3 \mathrm{H})$, 3.47-3.38 (m, $1 \mathrm{H}), 2.53-2.48(\mathrm{~m}, 1 \mathrm{H}), 2.29(\mathrm{q}, J=13.2 \mathrm{~Hz}, 1$ $\mathrm{H}), 1.82-1.65(\mathrm{~m}, 2 \mathrm{H}), 1.61-1.54(\mathrm{~m}, 1 \mathrm{H}) \mathrm{ppm} ;{ }^{13} \mathrm{C}\left\{{ }^{1} \mathrm{H}\right\} \mathrm{NMR}$ $\left(100 \mathrm{MHz}, \mathrm{CDCl}_{3}\right): \delta 169.0,142.1,140.9,134.5,129.4,129.2$, $129.1,128.3,128.1,128.0,127.1,126.7,77.4,77.1,76.7$, 73.4, 58.0, 56.0, 55.7, 53.0, 51.6, 34.2, $30.8 \mathrm{ppm}$; $v_{\max }($ neat $) / \mathrm{cm}^{-1}: 2981,1733,1245,1141,1031,697 \mathrm{~cm}^{-1}$; HRMS (ESI) m/z: [M] $]^{+}$calcd for $\mathrm{C}_{27} \mathrm{H}_{27}{ }^{35} \mathrm{ClNO}_{2} 432.1730$; found 432.1726; calcd for $\mathrm{C}_{27} \mathrm{H}_{27}{ }^{37} \mathrm{ClNO}_{2} 434.1701$; found 434.1696 .

$(4 S, 6 R, 8 R, 9 a S)$-8-bromo-6-phenethyl-4-phenylhexahydropyrido[2,1-c] $[1,4]$ oxazin-1(6H)-one (13a, Table 4 Entry 6). 
Following the general procedure $\mathbf{C}$, the title compound $\mathbf{1 3 a}$ was prepared from compound $\mathbf{8 a}(109 \mathrm{mg})$ and phenyl propionaldehyde as a white solid $(186 \mathrm{mg}, 90 \%)$. $[\alpha]_{\mathrm{D}^{26}}=+225.0(c$ 1.0, $\left.\mathrm{CHCl}_{3}\right)$; M.pt. $192-194{ }^{\circ} \mathrm{C} ;{ }^{1} \mathrm{H} \mathrm{NMR}\left(400 \mathrm{MHz}, \mathrm{CDCl}_{3}\right): \delta$ 7.40-7.14 (m, $8 \mathrm{H}), 7.11-7.05$ (m, $2 \mathrm{H}), 4.40-4.24$ (m, $4 \mathrm{H})$, 4.11 (dd, $J=3.2,9.2 \mathrm{~Hz}, 1 \mathrm{H}), 2.82-2.70(\mathrm{~m}, 2 \mathrm{H}), 2.51-2.27$ $(\mathrm{m}, 3 \mathrm{H}), 2.20-1.80(\mathrm{~m}, 3 \mathrm{H}), 1.63-1.54(\mathrm{~m}, 1 \mathrm{H}) \mathrm{ppm} ;{ }^{13} \mathrm{C}\left\{{ }^{1} \mathrm{H}\right\}$ NMR (100 MHz, $\left.\mathrm{CDCl}_{3}\right): \delta 168.9,141.4,135.3,129.3,128.6$, 128.5, 128.2, 126.1, 77.3, 77.1, 76.8, 73.9, 56.3, 55.8, 54.7, 44.7, 35.0, 34.7, 33.0, $32.6 \mathrm{ppm} ; v_{\max }$ (neat) $/ \mathrm{cm}^{-1}: 2981,1726$, 1453, 1242, 1133, $698 \mathrm{~cm}^{-1}$; HRMS (ESI) $\mathrm{m} / z:[\mathrm{M}]^{+}$calcd for $\mathrm{C}_{22} \mathrm{H}_{25}{ }^{79} \mathrm{BrNO}_{2}$ 414.1063; found 414.1063; calcd for $\mathrm{C}_{22} \mathrm{H}_{25}{ }^{81} \mathrm{BrNO}_{2}$ 416.1043; found 416.1042.

(4S,6S,8R,9aS)-6-Benzhydryl-8-bromo-4-phenylhexahydropyrido[2,1-c] [1,4] oxazin-1(6H)-one (13b, Table 4 Entry 7). Following the general procedure $\mathbf{C}$, the title compound 13b was prepared from compound $\mathbf{8 a}(109 \mathrm{mg})$ and diphenyl acetaldehyde as a white solid $(211 \mathrm{mg}, 80 \%)$. $[\alpha]_{\mathrm{D}}{ }^{26}=+183.0$ (c 1.0, $\mathrm{CHCl}_{3}$ ); M.pt. 201-203 ${ }^{\circ} \mathrm{C} ;{ }^{1} \mathrm{H}$ NMR (400 MHz, $\left.\mathrm{CDCl}_{3}\right): \delta$ 7.51-7.30 (m, $\left.4 \mathrm{H}\right), 7.28-7.13(\mathrm{~m}, 6 \mathrm{H}), 7.09-7.00$ (m, $3 \mathrm{H}), 6.98-6.90$ (m, $2 \mathrm{H}), 4.44-4.31$ (m, $3 \mathrm{H}), 4.13-3.97$ (m, $3 \mathrm{H}), 3.38-3.32(\mathrm{~m}, 1 \mathrm{H}), 2.61-2.43(\mathrm{~m}, 2 \mathrm{H}), 1.93-1.84$ (m, $2 \mathrm{H}) \mathrm{ppm} ;{ }^{13} \mathrm{C}\left\{{ }^{1} \mathrm{H}\right\} \mathrm{NMR}\left(100 \mathrm{MHz}, \mathrm{CDCl}_{3}\right): \delta 168.7$, 142.0, 140.9, 134.5, 129.9, 129.4, 129.2, 129.1, 128.3, 128.1, 128.0, 127.1, 126.7, 77.3, 77.1, 76.8, 76.5, 73.3, 58.6, 56.4, 56.0, 51.3, 44.0, 34.9, $31.7 \mathrm{ppm} ; v_{\max }$ (neat) $/ \mathrm{cm}^{-1}: 2933,1738$, 1454, 1228, 1021, $702 \mathrm{~cm}^{-1}$; HRMS (ESI) $\mathrm{m} / \mathrm{z}:[\mathrm{M}]^{+}$calcd for $\mathrm{C}_{27} \mathrm{H}_{27}{ }^{79} \mathrm{BrNO}_{2}$ 476.1220; found 476.1216; calcd for $\mathrm{C}_{27} \mathrm{H}_{27}{ }^{81} \mathrm{BrNO}_{2}$ 478.1200; found 478.1195.

(4S,6R,8R,9aS)-8-Hydroxy-4-phenyl-6-propylhexahydropyrido[2,1-c] [1,4] oxazin-1(6H)-one (14a, Table 4 Entry 8). Following the general procedure, $\mathbf{D}$, the title compound $\mathbf{1 4 a}$ was prepared from compound $\mathbf{8 a}(109 \mathrm{mg})$ and butanal as a colourless liquid $(127 \mathrm{mg}, 88 \%)$. $[\alpha]_{\mathrm{D}}{ }^{26}=+65.0($ c 1.0, $\left.\mathrm{CHCl}_{3}\right) ;{ }^{1} \mathrm{H}$ NMR $\left(400 \mathrm{MHz}, \mathrm{CDCl}_{3}\right): \delta$ 7.38-7.30 (m, $\left.5 \mathrm{H}\right)$, 4.35-4.22 (m, $3 \mathrm{H}), 4.02-3.95(\mathrm{~m}, 2 \mathrm{H}), 2.83-2.75$ (m, $1 \mathrm{H})$, 2.46 (bs, $1 \mathrm{H}), 2.16-1.96$ (m, 2H), 1.54-1.35 (m, $4 \mathrm{H}), 1.22-$ $1.09(\mathrm{~m}, 2 \mathrm{H}), 0.77(\mathrm{t}, J=5.6 \mathrm{~Hz}, 1 \mathrm{H}) \mathrm{ppm} ;{ }^{13} \mathrm{C}\left\{{ }^{1} \mathrm{H}\right\} \mathrm{NMR}$ $\left(100 \mathrm{MHz}, \mathrm{CDCl}_{3}\right): \delta 170.5,135.7,128.2,127.9,127.6,76.5$, 76.3, 76.0, 72.6, 63.6, 55.5, 53.6, 52.9, 33.2, 32.1, 31.9, 18.8, $13.2 \mathrm{ppm} ; v_{\max }($ neat $) / \mathrm{cm}^{-1}: 2955,2870,1732,1456,1330$, 1228, 1138, 761, $647 \mathrm{~cm}^{-1}$; HRMS (ESI) $\mathrm{m} / \mathrm{z}:[\mathrm{M}]^{+}$calcd for $\mathrm{C}_{17} \mathrm{H}_{24} \mathrm{O}_{3} \mathrm{~N} 290.1751$; found 290.1750. Compound 14a was also prepared using the same method starting from 8a (109 $\mathrm{mg}$ ) and butanal diethyl acetal $(73 \mathrm{mg}$ ) in $170 \mathrm{mg}$, $76 \%$ yield (Table 4 Entry 10). All data was in agreement with that presented above.

(4S,6R,8R,9aS)-8-hydroxy-4-phenyl-6-undecylhexahydropyrido[2,1-c] [1,4] oxazin-1(6H)-one (14b, Table 4 Entry 9). Following the general procedure, $\mathbf{D}$, the title compound 14b was prepared from compound 8a (109 mg) and dodecanal as a colourless liquid $(172 \mathrm{mg}, 90 \%)$. $[\alpha]_{\mathrm{D}}{ }^{26}=+187.0(c 1.0$, $\left.\mathrm{CHCl}_{3}\right) ;{ }^{1} \mathrm{H} \mathrm{NMR}\left(400 \mathrm{MHz}, \mathrm{CDCl}_{3}\right): \delta$ 7.40-7.30 (m, $\left.5 \mathrm{H}\right)$, 4.35-4.21 (m, $3 \mathrm{H}), 4.02-3.96(\mathrm{~m}, 2 \mathrm{H}), 2.83-2.76(\mathrm{~m}, 1 \mathrm{H})$, 2.19-1.96 (m, 2H), 1.54-1.45 (m, $2 \mathrm{H}), 1.38-1.05$ (m, $22 \mathrm{H})$, $0.87(\mathrm{t}, J=5.6 \mathrm{~Hz}, 1 \mathrm{H}) \mathrm{ppm} ;{ }^{13} \mathrm{C}\left\{{ }^{1} \mathrm{H}\right\} \mathrm{NMR}(100 \mathrm{MHz}$, $\left.\mathrm{CDCl}_{3}\right): \delta 171.4,136.6,129.0,128.7,128.4,77.3,77.1,76.8$, 73.4, 64.5, 56.6, 54.4, 53.9, 32.9, 31.9, 29.6, 29.4, 26.4, 22.7, $14.1 \mathrm{ppm} ; v_{\max }\left(\right.$ neat) $/ \mathrm{cm}^{-1}: 2923,2853,1739,1456,1230$, 1044, $762 \mathrm{~cm}^{-1}$; HRMS (ESI) $\mathrm{m} / z:[\mathrm{M}]^{+}$calcd for $\mathrm{C}_{25} \mathrm{H}_{40} \mathrm{O}_{3} \mathrm{~N}$ 402.3003; found 402.2999.

(4S,6R,8R,9aS)-6-Nonyl-1-oxo-4-phenyloctahydropyrido[2,1-c] $[1,4]$ oxazin-8-yl 4-methylbenzenesulfonate (15a, Table 4 Entry 11). Following the general procedure $\mathbf{C}$, the title compound 15a was prepared from compound 8a (109 $\mathrm{mg})$ and decanal as a white solid $(211 \mathrm{mg}, 80 \%) .[\alpha]_{\mathrm{D}}{ }^{26}=$ +65.0 (c 1.0, $\left.\mathrm{CHCl}_{3}\right)$; M.pt. 170-172 ${ }^{\circ} \mathrm{C} ;{ }^{1} \mathrm{H} \mathrm{NMR} \mathrm{(400} \mathrm{MHz,}$ $\left.\mathrm{CDCl}_{3}\right): \delta$ 7.80-7.74 (m, $\left.2 \mathrm{H}\right), 7.41-7.28(\mathrm{~m}, 7 \mathrm{H}), 4.78-4.71$ (m, $1 \mathrm{H}), 4.33-4.17$ (m, $3 \mathrm{H}), 4.00-3.94(\mathrm{~m}, 1 \mathrm{H}), 2.77-2.71$ (m, $1 \mathrm{H}), 2.44(\mathrm{~s}, 3 \mathrm{H}), 2.10-2.02(\mathrm{~m}, 1 \mathrm{H}), 1.65-1.42(\mathrm{~m}, 3 \mathrm{H})$, 1.34-1.02 (m, $15 \mathrm{H}), 0.88(\mathrm{t}, J=5.2 \mathrm{~Hz}, 1 \mathrm{H}) \mathrm{ppm} ;{ }^{13} \mathrm{C}\left\{{ }^{1} \mathrm{H}\right\}$ NMR (100 MHz, $\left.\mathrm{CDCl}_{3}\right): \delta 169.2,144.9,135.4,134.3,130.0$, 129.2, 128.6, 127.6, 77.3, 77.1, 76.8, 76.2, 73.8, 55.7, 54.3, 53.6, 31.9, 31.0, 29.9, 29.6, 29.4, 26.5, 22.7, 21.7, 14.2 ppm; $v_{\max }($ neat $) / \mathrm{cm}^{-1}: 2981,1733,1456,1232,940,816,698,551$ $\mathrm{cm}^{-1}$; HRMS (ESI) $\mathrm{m} / \mathrm{z}$ : [M] ${ }^{+}$calcd for $\mathrm{C}_{30} \mathrm{H}_{42} \mathrm{O}_{5} \mathrm{NS} 528.2778$; found 528.2768.

(4S,6R,8R,9aS)-1-oxo-4-phenyl-6-undecyloctahydropyrido[2,1-c] [1,4] oxazin-8-yl 4-methylbenzenesulfonate (15b, Table 4 Entry 12). Following the general procedure $\mathbf{C}$, the title compound $\mathbf{1 5 b}$ was prepared from compound 8a (109 $\mathrm{mg})$ and dodecanal as a white solid $(216 \mathrm{mg}, 78 \%) \cdot[\alpha]_{\mathrm{D}}{ }^{26}=$ +223.0 (c 1.0, $\mathrm{CHCl}_{3}$ ); M.pt. $178-180{ }^{\circ} \mathrm{C} ;{ }^{1} \mathrm{H}$ NMR $(400 \mathrm{MHz}$, $\left.\mathrm{CDCl}_{3}\right): \delta$ 7.80-7.74 (m, $\left.2 \mathrm{H}\right), 7.41-7.28(\mathrm{~m}, 7 \mathrm{H}), 4.78-4.68$ (m, $1 \mathrm{H}), 4.33-4.17$ (m, $3 \mathrm{H}), 4.00-3.93(\mathrm{~m}, 1 \mathrm{H}), 2.77-2.70$ (m, $1 \mathrm{H}), 2.44(\mathrm{~s}, 3 \mathrm{H}), 2.10-2.01(\mathrm{~m}, 1 \mathrm{H}), 1.65-1.43(\mathrm{~m}, 3 \mathrm{H})$, 1.34-1.02 (m, $20 \mathrm{H}), 0.88(\mathrm{t}, J=5.2 \mathrm{~Hz}, 1 \mathrm{H}) \mathrm{ppm} ;{ }^{13} \mathrm{C}\left\{{ }^{1} \mathrm{H}\right\}$ NMR $\left(100 \mathrm{MHz}, \mathrm{CDCl}_{3}\right): \delta 168.2,143.9,134.4,133.3,129.0$, 128.2, 127.5, 126.6, 76.3, 76.0, 75.8, 75.2, 72.8, 54.7, 53.3, 52.6, 30.9, 30.0, 28.9, 28.6, 28.4, 25.5, 21.7, 20.7, 13.1 ppm; $v_{\max }($ neat $) / \mathrm{cm}^{-1}: 2981,1734,1456,1190,1037,751,698 \mathrm{~cm}^{-1}$; HRMS (ESI) $m / z$ : [M] $]^{+}$calcd for $\mathrm{C}_{32} \mathrm{H}_{46} \mathrm{O}_{5} \mathrm{NS} 556.3091$; found 556.3081 .

\section{$N$-((4S,6R,8R,9aS)-6-nonyl-1-oxo-4-phenyloctahydro-} pyrido[2,1-c] $[1,4]$ oxazin-8-yl) acetamide (16a, Table 4 Entry 13). Following the general procedure $\mathbf{E}$, the title compound 16a was prepared from compound $8 \mathbf{8}(109 \mathrm{mg})$ and decanal as a semi solid $(155 \mathrm{mg}, 75 \%)$. $[\alpha]_{\mathrm{D}}{ }^{26}=+74.0(c 1.0$, $\left.\mathrm{CHCl}_{3}\right) ;{ }^{1} \mathrm{H}$ NMR $\left(400 \mathrm{MHz}, \mathrm{CDCl}_{3}\right): \delta$ 7.39-7.30 (m, $\left.5 \mathrm{H}\right)$, 5.94 (bs, $1 \mathrm{H}), 4.27-4.08$ (m, $5 \mathrm{H}), 2.72-2.63$ (m, $1 \mathrm{H}), 2.21-$ $2.19(\mathrm{~m}, 1 \mathrm{H}), 1.95(\mathrm{~s}, 3 \mathrm{H}), 1.62-1.49(\mathrm{~m}, 2 \mathrm{H}), 1.44-1.02(\mathrm{~m}$, $17 \mathrm{H}), 0.87(\mathrm{t}, J=6.8 \mathrm{~Hz}, 1 \mathrm{H}) \mathrm{ppm} ;{ }^{13} \mathrm{C}\left\{{ }^{1} \mathrm{H}\right\}$ NMR $(100$ $\left.\mathrm{MHz}_{\mathrm{CDCl}}\right): \delta 171.8,169.4,137.0,129.1,128.7,128.1$, 77.4, 77.1, 76.7, 73.0, 57.6, 54.4, 54.1, 42.6, 31.9, 31.7, 31.0, 29.6, 29.3, 26.1 23.6, 22.7, $14.2 \mathrm{ppm} ; \nu_{\max }$ (neat) $/ \mathrm{cm}^{-1}: 2980$, 1739, 1549, 1233, 1021, $702 \mathrm{~cm}^{-1}$; HRMS (ESI) $\mathrm{m} / \mathrm{z}:[\mathrm{M}]^{+}$ calcd for $\mathrm{C}_{25} \mathrm{H}_{39} \mathrm{O}_{3} \mathrm{~N}_{2}$ 415.2955; found 415.2952.

\section{$N$-((4S,6R,8R,9aS)-1-oxo-4-phenyl-6-undecyloctahydro-} pyrido[2,1-c] $[1,4]$ oxazin-8-yl) acetamide (16b, Table 4 Entry 141). Following the general procedure $\mathbf{E}$, the title compound 16b was prepared from compound 8a (109 $\mathrm{mg})$ and dodecanal as a semi solid (159 mg, $72 \%)$. $[\alpha]_{\mathrm{D}}{ }^{26}=+94.0(c 1.0$, $\left.\mathrm{CHCl}_{3}\right) ;{ }^{1} \mathrm{H} \mathrm{NMR}\left(400 \mathrm{MHz}, \mathrm{CDCl}_{3}\right): \delta$ 7.41-7.30 (m, $\left.5 \mathrm{H}\right)$, 5.94 (bs, $1 \mathrm{H}), 4.27-4.08$ (m, $5 \mathrm{H}), 2.72-2.63$ (m, $1 \mathrm{H}), 2.21-$ $2.14(\mathrm{~m}, 1 \mathrm{H}), 1.95(\mathrm{~s}, 3 \mathrm{H}), 1.47-1.02(\mathrm{~m}, 20 \mathrm{H}), 0.87$ (t, $J=$ $6.8 \mathrm{~Hz}, 1 \mathrm{H}) \mathrm{ppm} ;{ }^{13} \mathrm{C}\left\{{ }^{1} \mathrm{H}\right\} \mathrm{NMR}\left(100 \mathrm{MHz}, \mathrm{CDCl}_{3}\right): \delta 171.8$, 169.3, 137.0, 129.0, 128.7, 128.1, 77.4, 77.1, 76.7, 72.9, 57.7, 54.4, 54.1, 42.6, 31.9, 31.8, 31.1, 29.7, 29.6, 29.4, 26.0, 23.6, 22.7, $14.2 \mathrm{ppm} ; v_{\max }($ neat $) / \mathrm{cm}^{-1}: 2971,1742,1381,1152,958$, $600 \mathrm{~cm}^{-1}$; HRMS (ESI) m/z: [M] $]^{+}$calcd for $\mathrm{C}_{27} \mathrm{H}_{43} \mathrm{O}_{3} \mathrm{~N}_{2}$ 443.3268; found 443.3264 .

(4S,6R,7S,8S,9aS)-8-Chloro-7-methyl-6-phenethyl-4-phenylhexahydropyrido[2,1-c] $[1,4]$ oxazin-1 $(6 H)$-one $(17 a$, Table 5 Entry 1). Following the general procedure $\mathbf{C}$, the title compound 16a was prepared from compound $\mathbf{8 c}(116 \mathrm{mg})$ and 3-phenylpropionaldehyde as a white solid (176 mg, $92 \%$ ). 
$[\alpha]_{\mathrm{D}}{ }^{26}=+64.0\left(c\right.$ 1.0, $\left.\mathrm{CHCl}_{3}\right) ;$ M.pt. $128-130{ }^{\circ} \mathrm{C} ;{ }^{1} \mathrm{H} \mathrm{NMR}$ $\left(400 \mathrm{MHz}, \mathrm{CDCl}_{3}\right): \delta$ 7.39-7.31 (m, $\left.5 \mathrm{H}\right), 7.28-7.25(\mathrm{~m}, 2 \mathrm{H})$, 7.21-7.16 (m, 1 H), 7.14-7.10 (m, $2 \mathrm{H}), 4.44-4.34$ (m, $2 \mathrm{H})$, $4.27(\mathrm{dd}, J=2.4,6.0 \mathrm{~Hz}, 1 \mathrm{H}), 4.15(\mathrm{dd}, J=2.4,6.0 \mathrm{~Hz}, 1 \mathrm{H})$, 3.96 (dt, $J=4.4,4.8 \mathrm{~Hz}, 1 \mathrm{H}), 2.94-2.88(\mathrm{~m}, 1 \mathrm{H}), 2.70-2.65$ (m, $1 \mathrm{H}), 2.45-2.28(\mathrm{~m}, 3 \mathrm{H}), 1.98-1.92(\mathrm{~m}, 1 \mathrm{H}), 1.67-1.59$ $(\mathrm{m}, 1 \mathrm{H}), 0.86(\mathrm{~d}, J=5.2 \mathrm{~Hz}, 3 \mathrm{H}) \mathrm{ppm} ;{ }^{13} \mathrm{C}\left\{{ }^{1} \mathrm{H}\right\}$ NMR $(100$ $\left.\mathrm{MHz}_{1} \mathrm{CDCl}_{3}\right): \delta 169.1,141.9,135.2,129.3,129.2,128.8$, 128.6, 128.3, 126.1, 77.4, 77.1, 76.7, 73.9, 61.2, 59.3, 55.5, 55.3, 36.0, 35.0, 33.1, 26.6, $15.7 \mathrm{ppm} ; v_{\max }($ neat $) / \mathrm{cm}^{-1}: 2980$, 1604, 1452, 1042, 909, $595 \mathrm{~cm}^{-1}$; HRMS (ESI) $\mathrm{m} / \mathrm{z}:[\mathrm{M}]^{+}$calcd for $\mathrm{C}_{23} \mathrm{H}_{27}{ }^{35} \mathrm{ClNO}_{2}$ 384.1730; found 384.1734; calcd for $\mathrm{C}_{23} \mathrm{H}_{27}{ }^{37} \mathrm{ClNO}_{2}$ 386.1701; found 386.1709.

(4S,6R,7S,8S,9aS)-8-Bromo-7-methyl-6-phenethyl-4-phenylhexahydropyrido[2,1-c] $[1,4]$ oxazin-1 $(6 H)$-one $(17 b$, Table 5 Entry 1). Following the general procedure $\mathbf{C}$, the title compound 16b was prepared from compound $\mathbf{8 c}(116 \mathrm{mg})$ and 3-phenylpropionaldehyde as a white solid (194 mg, $91 \%)$. $[\alpha]_{\mathrm{D}}{ }^{26}=+183.0\left(c\right.$ 1.0, $\left.\mathrm{CHCl}_{3}\right)$; M.pt. 136-138 ${ }^{\circ} \mathrm{C} ;{ }^{1} \mathrm{H} \mathrm{NMR}$ (400 MHz, $\left.\mathrm{CDCl}_{3}\right): \delta$ 7.41-7.26 (m, $\left.7 \mathrm{H}\right), 7.21-7.16(\mathrm{~m}, 1 \mathrm{H})$, 7.13-7.08 (m, $2 \mathrm{H}), 4.51-4.35(\mathrm{~m}, 2 \mathrm{H}), 4.27$ (dd, $J=3.6,7.2$ $\mathrm{Hz}, 1 \mathrm{H}), 4.19-4.10$ (m, $2 \mathrm{H}), 2.96-2.84$ (m, $1 \mathrm{H}), 2.70-2.50$ (m, $2 \mathrm{H}), 2.35-2.26(\mathrm{~m}, 1 \mathrm{H}), 2.11-2.01(\mathrm{~m}, 1 \mathrm{H}), 1.71-1.58$ $(\mathrm{m}, 2 \mathrm{H}), 0.87(\mathrm{~d}, J=6.8 \mathrm{~Hz}, 3 \mathrm{H}) \mathrm{ppm} ;{ }^{13} \mathrm{C}\left\{{ }^{1} \mathrm{H}\right\} \mathrm{NMR}(100$ $\left.\mathrm{MHz} \mathrm{CDCl}_{3}\right): \delta 168.8,141.9,135.1,129.3,128.8,128.6$, 128.3, 126.1, 77.4, 77.1, 76.7, 73.9, 59.4, 55.9, 55.5, 54.3, 36.3, 36.2, 33.0, 26.4, $17.6 \mathrm{ppm} ; v_{\max }$ (neat) $/ \mathrm{cm}^{-1}: 2981,1739$, 1235, 1064, 766, 635, $528 \mathrm{~cm}^{-1}$; HRMS (ESI) $\mathrm{m} / \mathrm{z}:[\mathrm{M}]^{+}$calcd for $\mathrm{C}_{23} \mathrm{H}_{27}{ }^{79} \mathrm{BrNO}_{2} 428.1225$; found 428.1221; calcd for $\mathrm{C}_{23} \mathrm{H}_{27}{ }^{81} \mathrm{BrNO}_{2} 430.1205$; found 430.1201.

(4S,6R,7S,8S,9aS)-8-Hydroxy-7-methyl-6-phenethyl-4-phenylhexahydropyrido[2,1-c] $[1,4]$ oxazin-1 $(6 H)$-one $(17 c$, Table 5 Entry 1). Following the general procedure, $\mathbf{C}$, the $t i$ tle compound 16c was prepared from compound $\mathbf{8 c}(116 \mathrm{mg})$ and phenyl propionaldehyde as a white solid $(151 \mathrm{mg}, 83 \%)$. $[\alpha]_{\mathrm{D}}^{26}=+82.0\left(\right.$ c 1.0, $\left.\mathrm{CHCl}_{3}\right)$; M.pt. $132-134{ }^{\circ} \mathrm{C} ;{ }^{1} \mathrm{H}$ NMR (400 MHz, $\left.\mathrm{CDCl}_{3}\right): \delta$ 7.39-7.31 (m, $\left.5 \mathrm{H}\right), 7.28-7.25(\mathrm{~m}, 2 \mathrm{H})$, 7.19-7.16 (m, $1 \mathrm{H})$, 7.14-7.08 (m, $2 \mathrm{H}), 4.44-4.34$ (m, $2 \mathrm{H})$, $4.27(\mathrm{dd}, J=2.8,5.6 \mathrm{~Hz}, 1 \mathrm{H}), 4.13(\mathrm{dd}, J=2.8,5.6 \mathrm{~Hz}, 1 \mathrm{H})$, $3.65(\mathrm{dt}, J=4.0,4.4 \mathrm{~Hz}, 1 \mathrm{H}), 2.94-2.87(\mathrm{~m}, 1 \mathrm{H}), 2.67-2.60$ (m, $1 \mathrm{H}), 2.34-2.27(\mathrm{~m}, 1 \mathrm{H}), 2.20-2.15(\mathrm{~m}, 1 \mathrm{H}), 2.06-1.98$ $(\mathrm{m}, 1 \mathrm{H}), 1.76-1.66(\mathrm{~m}, 1 \mathrm{H}), 1.62-1.57(\mathrm{~m}, 1 \mathrm{H}), 0.81(\mathrm{~d}, J=$ $5.6 \mathrm{~Hz}, 3 \mathrm{H}), \mathrm{ppm} ;{ }^{13} \mathrm{C}\left\{{ }^{1} \mathrm{H}\right\} \mathrm{NMR}\left(100 \mathrm{MHz}, \mathrm{CDCl}_{3}\right): \delta$ 170.4, 142.3, 135.7, 129.2, 129.0, 128.7, 128.5, 128.3, 125.9, 77.3, 77.1, 76.8, 73.9, 70.01 59.0, 55.6, 54.6, 34.9, 33.3, 33.1, 27.4, $13.9 \mathrm{ppm} ; v_{\max }($ neat $) / \mathrm{cm}^{-1}: 2981,1701,1245,1059,699$ $\mathrm{cm}^{-1}$; HRMS (ESI) $\mathrm{m} / z$ : $[\mathrm{M}]^{+}$calcd for $\mathrm{C}_{23} \mathrm{H}_{28} \mathrm{NO}_{3} 366.2069$; found 366.2072.

(4S,6R,7S,8aS)-7-(2-Chloropropan-2-yl)-6-phenethyl-4phenylhexahydro-1H-pyrrolo[2,1-c] $[1,4]$ oxazin-1-one (18a, Table 5 Entry 2). Following the general procedure $\mathbf{C}$, the title compound 17a was prepared from compound $\mathbf{8 e}$ (123 $\mathrm{mg}$ ) and phenyl propionaldehyde as a white solid (125 mg, 63 \%). $[\alpha]_{\mathrm{D}}^{26}=+32.0\left(\right.$ ( $\left.1.0, \mathrm{CHCl}_{3}\right)$; M.pt. $152-154{ }^{\circ} \mathrm{C} ;{ }^{1} \mathrm{H}$ NMR $\left(400 \mathrm{MHz}, \mathrm{CDCl}_{3}\right): \delta$ 7.49-7.32 (m, $\left.5 \mathrm{H}\right), 7.21-7.08(\mathrm{~m}, 3 \mathrm{H})$, 6.86-6.81 (m, 2 H), 4.40-4.33 (m, $1 \mathrm{H}), 4.28-4.160$ (m, $4 \mathrm{H})$, 3.10-3.04 (m, $1 \mathrm{H}), 2.60$ (q, $J=4.8,8 \mathrm{~Hz}, 1 \mathrm{H}), 2.44-2.37$ (m, $3 \mathrm{H}), 2.23-2.11(\mathrm{~m}, 3 \mathrm{H}), 1.73-1.64(\mathrm{~m}, 1 \mathrm{H}), 1.57$ (s, $3 \mathrm{H})$, $1.48(\mathrm{~s}, 3 \mathrm{H}) \mathrm{ppm} ;{ }^{13} \mathrm{C}\left\{{ }^{1} \mathrm{H}\right\} \mathrm{NMR}\left(100 \mathrm{MHz}, \mathrm{CDCl}_{3}\right): \delta 171.2$, 142.3, 137.4, 129.1, 128.8, 128.4, 128.3, 128.2, 125.7, 77.4, 77.1, 76.7, 72.9, 72.3, 66.2, 60.6, 59.6, 56.5, 39.0, 32.8, 32.5, $32.1,32.0 \mathrm{ppm} ; v_{\max }($ neat $) / \mathrm{cm}^{-1}: 2981,1752,1384,1156,698$ $\mathrm{cm}^{-1}$; HRMS (ESI) $\mathrm{m} / z$ : [M] ${ }^{+}$calcd for $\mathrm{C}_{24} \mathrm{H}_{29}{ }^{35} \mathrm{ClNO}_{2}$
398.1886; found 398.1884; calcd for $\mathrm{C}_{24} \mathrm{H}_{29}{ }^{37} \mathrm{ClNO}_{2} 400.1857$; found 400.1861 .

(4S,6R,7S,8aS)-7-(2-Hydroxypropan-2-yl)-6-phenethyl-4phenylhexahydro-1H-pyrrolo[2,1-c] $[1,4]$ oxazin-1-one (18b, Table 5 Entry 2). Following the general procedure, A, the title compound $\mathbf{1 7 b}$ was prepared from compound $\mathbf{8 e}$ (123 $\mathrm{mg}$ ) and 3-phenylpropionaldehyde as a white solid (144 mg, $76 \%$ ). $[\alpha]_{\mathrm{D}}{ }^{26}=+136.0$ (c 1.0, $\left.\mathrm{CHCl}_{3}\right)$; M.pt. $134-136{ }^{\circ} \mathrm{C} ;{ }^{1} \mathrm{H}$ NMR (400 MHz, $\left.\mathrm{CDCl}_{3}\right): \delta$ 7.49-7.34 (m, $\left.5 \mathrm{H}\right), 7.21-7.08(\mathrm{~m}$, $3 \mathrm{H}), 6.77-6.69$ (m, $2 \mathrm{H}), 4.35-4.12(\mathrm{~m}, 3 \mathrm{H}), 4.06(\mathrm{dd}, J=4.0$, $7.6 \mathrm{~Hz}, 1 \mathrm{H}), 3.06(\mathrm{q}, J=5.2 \mathrm{~Hz}, 1 \mathrm{H}), 2.52-2.25(\mathrm{~m}, 2 \mathrm{H})$, 2.01-1.94 (m, $1 \mathrm{H}), 1.71-1.44$ (m, $3 \mathrm{H}), 1.28$ (s, $3 \mathrm{H}), 1.14$ (s, $3 \mathrm{H}) \mathrm{ppm} ;{ }^{13} \mathrm{C}\left\{{ }^{1} \mathrm{H}\right\} \mathrm{NMR}\left(100 \mathrm{MHz}, \mathrm{CDCl}_{3}\right): \delta 173.1,142.5$, 138.8, 128.8, 128.4, 128.2, $125.677 .42,77.1,76.8,72.1,71.2$, 66.6, 61.4, 60.2, 52.7, 37.8, 31.2, 29.4, 28.9 ppm;

$v_{\max }($ neat $) / \mathrm{cm}^{-1}: 2980,1667,1453,1155,1029,646 \mathrm{~cm}^{-1}$; HRMS (ESI) $m / z:[\mathrm{M}]^{+}$calcd for $\mathrm{C}_{24} \mathrm{H}_{30} \mathrm{NO}_{3} 380.2225$; found 380.2221 .

(4S,6R,7S,8aS)-7-(2-Hydroxypropan-2-yl)-4-phenyl-6propylhexahydro-1H-pyrrolo[2,1-c] $[1,4]$ oxazin-1-one (18c, Table 5 Entry 3). Following the general procedure, A, the title compound $\mathbf{1 7} \mathbf{c}$ was prepared from compound $\mathbf{8 e}$ (123 $\mathrm{mg})$ and butanal as a white solid $(114 \mathrm{mg}, 72 \%)$. $[\alpha]_{\mathrm{D}}{ }^{26}=$ +182.0 (c 1.0, $\mathrm{CHCl}_{3}$ ); M.pt. 140-142 ${ }^{\circ} \mathrm{C} ;{ }^{1} \mathrm{H}$ NMR (400 MHz, $\left.\mathrm{CDCl}_{3}\right): \delta$ 7.49-7.28 (m, $\left.5 \mathrm{H}\right), 4.29-4.15(\mathrm{~m}, 2 \mathrm{H}), 4.07-4.02$ $(\mathrm{m}, 2 \mathrm{H}), 2.96(\mathrm{q}, J=5.2 \mathrm{~Hz}, 1 \mathrm{H}), 2.40-2.25(\mathrm{~m}, 2 \mathrm{H}), 1.87-$ $1.81(\mathrm{~m}, 1 \mathrm{H}), 1.41(\mathrm{bs}, 1 \mathrm{H}), 1.33-1.06(\mathrm{~m}, 10 \mathrm{H}), 0.54(\mathrm{t}, J=$ $7.2 \mathrm{~Hz}, 3 \mathrm{H}) \mathrm{ppm} ;{ }^{13} \mathrm{C}\left\{{ }^{1} \mathrm{H}\right\} \mathrm{NMR}\left(100 \mathrm{MHz}, \mathrm{CDCl}_{3}\right): \delta 172.8$, 138.5, 128.6, 128.5, 128.3, 77.4, 77.1, 76.7, 72.4, 71.1, 65.9, $60.7,60.5,53.3,38.5,30.1,29.5,29.0,18.3,13.9$ ppm; $v_{\max }($ neat $) / \mathrm{cm}^{-1}: 2981,1733,1355,1111,1017,698 \mathrm{~cm}^{-1}$; HRMS (ESI) $m / z$ : [M] $]^{+}$calcd for $\mathrm{C}_{19} \mathrm{H}_{28} \mathrm{NO}_{3} 318.2069$; found 318.2079.

(4S,6R,7R,8aS)-6-Phenethyl-4-phenyl-7-(prop-1-en-2-yl) hexahydro-1H-pyrrolo[2,1-c] [1,4] oxazin-1-one: (18d, Table 5, Entry 4). Following the general procedure $\mathbf{E}$, the title compound 17d was prepared from compound $8 \mathbf{e}(123 \mathrm{mg})$ and phenyl propionaldehyde in presence of catalytic amount of Sc $(\text { OTf })_{3}$ as a white solid $(90 \mathrm{mg}, 51 \%)$. $[\alpha]_{\mathrm{D}}{ }^{26}=-63.0(c 1.0$, $\mathrm{CHCl}_{3}$ ); M.pt. $168-170{ }^{\circ} \mathrm{C} ;{ }^{1} \mathrm{H}$ NMR (400 MHz, $\left.\mathrm{CDCl}_{3}\right): \delta$ 7.51-7.32 (m, $5 \mathrm{H}), 7.16-7.04(\mathrm{~m}, 3 \mathrm{H}), 6.58-6.53(\mathrm{~m}, 2 \mathrm{H})$, 4.87-4.82 (m, $2 \mathrm{H}), 4.28-4.16(\mathrm{~m}, 3 \mathrm{H}), 3.91-3.84(\mathrm{~m}, 1 \mathrm{H})$, 2.83-2.70 (m, $2 \mathrm{H}), 2.44-2.32(\mathrm{~m}, 3 \mathrm{H}), 1.73-1.62(\mathrm{~m}, 2 \mathrm{H})$, 1.55 (s, $3 \mathrm{H}) \mathrm{ppm} ;{ }^{13} \mathrm{C}\left\{{ }^{1} \mathrm{H}\right\} \mathrm{NMR}\left(100 \mathrm{MHz}, \mathrm{CDCl}_{3}\right): \delta 173.4$, 144.2, 142.3, 139.3, 128.9, 128.3, 128.1, 128.1, 127.8, 125.5, 113.1, 77.4, 77.1, 76.7, 71.2, 68.1, 63.1, 58.5, 49.8, 33.4, 31.0, 29.8, 19.2. ppm; $v_{\max }($ neat $) / \mathrm{cm}^{-1}: 2981,1756,1454,1137$, $1071,699 \mathrm{~cm}^{-1}$; HRMS (ESI) $\mathrm{m} / z$ : [M] $]^{+}$calcd for $\mathrm{C}_{24} \mathrm{H}_{28} \mathrm{NO}_{2}$ 362.2120; found 362.2122.

(4S,6R,8R,9aS)-8-Chloro-8-methyl-6-phenethyl-4-phenylhexahydropyrido[2,1-c] $[1,4]$ oxazin-1 $(6 H)$-one (19a, Table 5 Entry 5). Following the general procedure $\mathbf{C}$, the title compound 18a was prepared from compound $\mathbf{8 d}(116 \mathrm{mg})$ and 3phenylpropionaldehyde as a white solid $(145 \mathrm{mg}, 76 \%)$. $[\alpha]_{\mathrm{D}}{ }^{26}$ $=+162.0\left(\right.$ c 1.0, $\left.\mathrm{CHCl}_{3}\right)$; M.pt. $138-140{ }^{\circ} \mathrm{C} ;{ }^{1} \mathrm{H}$ NMR $(400$ $\left.\mathrm{MHz}, \mathrm{CDCl}_{3}\right): \delta$ 7.49-7.42 (m, $\left.2 \mathrm{H}\right), 7.40-7.24(\mathrm{~m}, 5 \mathrm{H}), 7.22-$ $7.15(\mathrm{~m}, 1 \mathrm{H}), 7.14-7.08(\mathrm{~m}, 2 \mathrm{H}), 4.75-4.55(\mathrm{~m}, 2 \mathrm{H}), 4.40(\mathrm{t}$, $J=4.4 \mathrm{~Hz}, 1 \mathrm{H}), 3.76(\mathrm{t}, J=5.6 \mathrm{~Hz}, 1 \mathrm{H}), 3.30-3.23(\mathrm{~m}, 1 \mathrm{H})$, $2.88-2.80(\mathrm{~m}, 1 \mathrm{H}), 2.66(\mathrm{t}, J=8.8 \mathrm{~Hz}, 1 \mathrm{H}), 2.17-2.11(\mathrm{~m}, 1$ $\mathrm{H}), 1.98-1.71(\mathrm{~m}, 3 \mathrm{H}), 1.70(\mathrm{~s}, 3 \mathrm{H}) \mathrm{ppm} ;{ }^{13} \mathrm{C}\left\{{ }^{1} \mathrm{H}\right\}$ NMR $(100$ $\left.\mathrm{MHz}, \mathrm{CDCl}_{3}\right): \delta 170.1,141.7,137.3,129.1,128.6,128.5$, 128.3, 127.9, 126.2, 67.6, 67.4, 54.9, 53.5, 52.9, 43.4, 38.9, $35.2,33.0,31.6 \mathrm{ppm} ; v_{\max }($ neat $) / \mathrm{cm}^{-1}: 2971,1740,1382,1151$, 
953, $700 \mathrm{~cm}^{-1}$; HRMS (ESI) $\mathrm{m} / \mathrm{z}:[\mathrm{M}]^{+}$calcd for $\mathrm{C}_{23} \mathrm{H}_{27}{ }^{35} \mathrm{ClNO}_{2} 384.1730$; found 384.1734; calcd for $\mathrm{C}_{23} \mathrm{H}_{27}{ }^{37} \mathrm{ClNO}_{2}$ 386.1701; found 386.1709.

(4S,6R,8R,9aS)-8-Chloro-8-methyl-4-phenyl-6-propylhexahydropyrido[2,1-c] $[1,4]$ oxazin-1 $(6 H)$-one $(19 b$, Table 5

Entry 6). Following the general procedure $\mathbf{C}$, the title compound $\mathbf{1 8 b}$ was prepared from compound $\mathbf{8 d}(116 \mathrm{mg})$ and butanal as a white solid $(115 \mathrm{mg}, 72 \%)$. $[\alpha]_{\mathrm{D}}^{26}=+126.0(c 1.0$, $\left.\mathrm{CHCl}_{3}\right)$; M.pt. $126-128{ }^{\circ} \mathrm{C} ;{ }^{1} \mathrm{H}$ NMR $\left(400 \mathrm{MHz}, \mathrm{CDCl}_{3}\right): \delta$ 7.49-7.42 (m, $2 \mathrm{H}), 7.40-7.27$ (m, $3 \mathrm{H}), 4.80$ (dd, $J=4.4,7.6$ $\mathrm{Hz}, 1 \mathrm{H}), 4.62$ (dd, $J=4.8,7.7 \mathrm{~Hz}, 1 \mathrm{H}), 4.39$ (t, $J=4.4 \mathrm{~Hz}, 1$ $\mathrm{H}), 3.72(\mathrm{t}, J=5.6 \mathrm{~Hz}, 1 \mathrm{H}), 3.23-3.13(\mathrm{~m}, 1 \mathrm{H}), 2.88-2.79(\mathrm{~m}$, $1 \mathrm{H}), 2.10-2.03(\mathrm{~m}, 1 \mathrm{H}), 1.87(\mathrm{dd}, J=6.0 \mathrm{~Hz}, 1 \mathrm{H}), 1.67(\mathrm{~s}, 3$ $\mathrm{H}), 1.65-1.59(\mathrm{~m}, 2 \mathrm{H}), 1.48-1.36(\mathrm{~m}, 3 \mathrm{H}), 0.87(\mathrm{t}, J=7.2 \mathrm{~Hz}$, $1 \mathrm{H}) \mathrm{ppm} ;{ }^{13} \mathrm{C}\left\{{ }^{1} \mathrm{H}\right\} \mathrm{NMR}\left(100 \mathrm{MHz}, \mathrm{CDCl}_{3}\right): \delta 170.2,137.6$, 129.0, 128.3, 127.8, 77.4, 77.1, 76.7, 67.7, 67.6, 54.8, 53.5, $52.9,43.5,38.9,35.4,33.0,18.4,14.4 \mathrm{ppm} ; v_{\max }($ neat $) / \mathrm{cm}^{-1}$ : 2965, 1723, 1367, 1148, 948, $688 \mathrm{~cm}^{-1}$; HRMS (ESI) $\mathrm{m} / \mathrm{z}$ : $[\mathrm{M}]^{+}$calcd for $\mathrm{C}_{18} \mathrm{H}_{25}{ }^{35} \mathrm{ClNO}_{2}$ 322.1573; found 322.1572; calcd for $\mathrm{C}_{18} \mathrm{H}_{25}{ }^{37} \mathrm{ClNO}_{2}$ 324.1544; found 324.1551.

(4S,6R,8R,9aS)-8-Hydroxy-8-methyl-6-phenethyl-4-phenylhexahydropyrido[2,1-c] $[1,4]$ oxazin-1(6H)-one (19c, Table 5 Entry 7). Following the general procedure, A, the title compound 18c was prepared from compound $8 \mathbf{d}(116 \mathrm{mg})$ and phenyl propionaldehyde as a white solid (146 mg, $80 \%)$. $[\alpha]_{\mathrm{D}}{ }^{26}=+56.0\left(\right.$ c 1.0, $\left.\mathrm{CHCl}_{3}\right)$; M.pt. 136-134 ${ }^{\circ} \mathrm{C} ;{ }^{1} \mathrm{H}$ NMR $\left(400 \mathrm{MHz}, \mathrm{CDCl}_{3}\right): \delta$ 7.46-7.29 (m, $\left.5 \mathrm{H}\right), 7.19-7.07(\mathrm{~m}, 3 \mathrm{H})$, 6.79-6.74 (m, $2 \mathrm{H}), 4.52$ (q, $J=10.8 \mathrm{~Hz}, 1 \mathrm{H}), 4.29-4.20$ (m, 2 H), 3.99 (dd, $J=2.4,4.0 \mathrm{~Hz}, 1 \mathrm{H}), 3.25$ (bs, $1 \mathrm{H}), 3.12-3.05$ (m, $1 \mathrm{H}), 2.51-2.40$ (m, $2 \mathrm{H}), 1.94-1.47$ (m, $3 \mathrm{H}), 1.32$ (s, $3 \mathrm{H})$ ppm; ${ }^{13} \mathrm{C}\left\{{ }^{1} \mathrm{H}\right\}$ NMR $\left(100 \mathrm{MHz}, \mathrm{CDCl}_{3}\right): \delta 175.1,142.1$, 139.4, 129.0, 128.3, 128.2, 128.1, 127.1, 125.8, 77.4, 77.1, 76.7, 69.6, 66.5, 60.1, 55.3, 53.8, 43.2, 36.3, 35.6, 31.3, 30.5 ppm; $v_{\max }($ neat $) / \mathrm{cm}^{-1}: 2981,1732,1391,1115,751,697 \mathrm{~cm}^{-1}$; HRMS (ESI) $m / z$ : [M] $]^{+}$calcd for $\mathrm{C}_{23} \mathrm{H}_{28} \mathrm{NO}_{3}$ 366.2069; found 366.2071 .

(4S,6R,8R,9aS)-8-Hydroxy-8-methyl-4-phenyl-6propylhexahydropyrido[2,1-c] $[1,4]$ oxazin-1(6H)-one (19d, Table 5 Entry 8). Following the general procedure, A, the $t i$ tle compound 18d was prepared from compound $\mathbf{8 d}$ (116 mg) and butanal as a white solid $(114 \mathrm{mg}, 75 \%)$. $[\alpha]_{\mathrm{D}}{ }^{26}=+320.0$ (c 1.0, $\mathrm{CHCl}_{3}$ ); M.pt. $128-130{ }^{\circ} \mathrm{C} ;{ }^{1} \mathrm{H}$ NMR (400 MHz, $\left.\mathrm{CDCl}_{3}\right): \delta$ 7.46-7.25 (m, $\left.4 \mathrm{H}\right), 7.31-7.26(\mathrm{~m}, 1 \mathrm{H}), 4.60-4.51$ (m, $1 \mathrm{H}), 4.33-4.20(\mathrm{~m}, 2 \mathrm{H}), 3.95-3.90(\mathrm{~m}, 1 \mathrm{H}), 3.24$ (m, 1 H), 2.96-2.90 (m, 1 H), 2.462.38 (m, $1 \mathrm{H}), 1.881 .70(\mathrm{~m}, 2 \mathrm{H})$, 1.50-1.36 (m, 2 H), 1.28 (s, $3 \mathrm{H}), 1.22-1.12(\mathrm{~m}, 2 \mathrm{H}), 1.32(\mathrm{t}, J$ $=6.8 \mathrm{~Hz}, 3 \mathrm{H}) \mathrm{ppm} ;{ }^{13} \mathrm{C}\left\{{ }^{1} \mathrm{H}\right\} \mathrm{NMR}\left(100 \mathrm{MHz}, \mathrm{CDCl}_{3}\right): \delta$ 175.2, 139.7, 128.8, 127.9, 127.0, 77.4, 77.1, 76.8, 69.6, 66.5, 59.9, 55.4, 53.7, 43.3, 36.3, 35.8, 31.3, 17.7, $14.2 \mathrm{ppm}$; $V_{\max }($ neat $) / \mathrm{cm}^{-1}: 2956,2872,1717,1399,1123,754 \mathrm{~cm}^{-1}$; HRMS (ESI) $m / z$ : [M] ${ }^{+}$calcd for $\mathrm{C}_{18} \mathrm{H}_{26} \mathrm{NO}_{3}$ 304.1912; found 304.1917.

(4S,6R,9aS)-8-chloro-6-phenethyl-4-phenyl-3,4,9,9a-tetrahydropyrido[2,1-c] $[1,4]$ oxazin-1(6H)-one (20a, Table 5 Entry 9). Following the general procedure $\mathbf{C}$, the title compound 19a was prepared from compound $\mathbf{8 b}(107 \mathrm{mg})$ and 3phenylpropionaldehyde as a semi solid $(128 \mathrm{mg}, 70 \%)$. [ $\alpha]_{\mathrm{D}}{ }^{26}$ $=-83.0\left(c 1.0, \mathrm{CHCl}_{3}\right) ;{ }^{1} \mathrm{H} \mathrm{NMR}\left(400 \mathrm{MHz}, \mathrm{CDCl}_{3}\right): \delta 7.37-$ 7.29 (m, 5 H), 7.23-7.11 (m, 3 H), 7.04-6.98 (m, 2 H), 5.77$5.73(\mathrm{~m}, 1 \mathrm{H}), 4.37-4.22(\mathrm{~m}, 3 \mathrm{H}), 4.11(\mathrm{dd}, J=5.2 \mathrm{~Hz}, 1 \mathrm{H})$, 3.13-3.05 (m, $1 \mathrm{H}), 2.90-2.79(\mathrm{~m}, 1 \mathrm{H}), 2.72-2.41$ (m, $3 \mathrm{H})$, 1.78-1.62 (m, $1 \mathrm{H}) \mathrm{ppm} ;{ }^{13} \mathrm{C}\left\{{ }^{1} \mathrm{H}\right\}$ NMR (100 MHz, $\left.\mathrm{CDCl}_{3}\right): \delta$ 169.2, 141.4, 135.9, 129.2, 129.0, 128.5, 128.5, 128.2, 127.5,
126.0, 125.6, 77.4, 77.1, 76.7, 73.4, 57.2, 56.0, 53.8, 35.9, $32.4,30.1 \mathrm{ppm} ; v_{\max }($ neat $) / \mathrm{cm}^{-1}: 2958,1746,1454,1121,1040$, 747, $660 \mathrm{~cm}^{-1}$; HRMS (ESI) $\mathrm{m} / z:[\mathrm{M}]^{+}$calcd for $\mathrm{C}_{22} \mathrm{H}_{23}{ }^{35} \mathrm{ClNO}_{2}$ 368.1417; found 368.1418; calcd for $\mathrm{C}_{22} \mathrm{H}_{23}{ }^{37} \mathrm{ClNO}_{2}$ 370.1388; found 370.1397.

(4S,6R,9aS)-8-bromo-6-phenethyl-4-phenyl-3,4,9,9a-tetrahydropyrido[2,1-c] $[1,4]$ oxazin-1 $(6 H)$-one $(20 b$, Table 5 Entry 9). Following the general procedure $\mathbf{C}$, the title compound 19b was prepared from compound $\mathbf{8 b}(107 \mathrm{mg})$ and 3phenylpropionaldehyde as a semi solid (146 mg, $71 \%)$. $[\alpha]_{\mathrm{D}}^{26}$ $=-232.0\left(c 1.0, \mathrm{CHCl}_{3}\right) ;{ }^{1} \mathrm{H} \mathrm{NMR}\left(400 \mathrm{MHz}, \mathrm{CDCl}_{3}\right): \delta 7.37-$ 7.29 (m, $5 \mathrm{H}), 7.23-7.11(\mathrm{~m}, 3 \mathrm{H}), 7.04-6.98$ (m, $2 \mathrm{H}), 6.01-$ $5.92(\mathrm{~m}, 1 \mathrm{H}), 4.37-4.26(\mathrm{~m}, 3 \mathrm{H}), 4.11(\mathrm{dd}, J=5.2 \mathrm{~Hz}, 1 \mathrm{H})$, 3.05-2.91 (m, $2 \mathrm{H}), 2.74-2.66(\mathrm{~m}, 2 \mathrm{H}), 2.47-2.40(\mathrm{~m}, 1 \mathrm{H})$, 1.78-1.64 (m, $2 \mathrm{H}) \mathrm{ppm} ;{ }^{13} \mathrm{C}\left\{{ }^{1} \mathrm{H}\right\} \mathrm{NMR}\left(100 \mathrm{MHz}, \mathrm{CDCl}_{3}\right): \delta$ 169.2, 141.4, 135.9, 129.2, 129.1, 128.5, 128.5, 128.2, 127.5, 126.0, 125.6, 77.4, 77.1, 76.7, 73.4, 57.1, 56.0, 53.8, 35.9, $32.4,30.1 \mathrm{ppm} ; v_{\max }\left(\right.$ neat) $/ \mathrm{cm}^{-1}: 2981,1727,1454,1249$, 1137, 700, $594 \mathrm{~cm}^{-1}$; HRMS (ESI) $\mathrm{m} / \mathrm{z}:[\mathrm{M}]^{+}$calcd for $\mathrm{C}_{22} \mathrm{H}_{23}{ }^{79} \mathrm{BrNO}_{2}$ 412.0912; found 414.0907; calcd for $\mathrm{C}_{22} \mathrm{H}_{23}{ }^{81} \mathrm{BrNO}_{2}$ 414.0892; found 414.0918.

General procedure F: Hydrogenation reaction: The compound $(0.5 \mathrm{mmol})$ was dissolved in ethanol $(10 \mathrm{~mL})$ and exhaustively $(14 \mathrm{~h})$ hydrogenated with double pressured balloon over $10 \% \mathrm{Pd}-\mathrm{C}(0.1 \mathrm{~g})$. The catalyst was removed by filtration and the filtrate concentrated. The resulting crude compound was washed with Ether twice for removal of non-polar impurities and dried to give pure white solid.

General procedure G: Hydrogenation reaction: The compound $(0.5 \mathrm{mmol})$ was dissolved in ethanol $(10 \mathrm{~mL})$ containing TFA ( 1 equiv) at room temperature and exhaustively (14 h) hydrogenated with double pressured balloon over $10 \%$ $\mathrm{Pd}(\mathrm{OH})_{2}(0.1 \mathrm{~g})$. The catalyst was removed by filtration and the filtrate concentrated. The resulting crude compound was washed with Ether twice for removal of non-polar impurities and dried to give pure white solid.

General procedure H: Hydrogenation reaction: The compound $(0.5 \mathrm{mmol})$ was dissolved in ethanolic $\mathrm{HCl}(10 \mathrm{~mL})$ at room temperature and exhaustively $(14 \mathrm{~h})$ hydrogenated with double pressured balloon over $10 \% \mathrm{Pd}(\mathrm{OH})_{2}(0.1 \mathrm{~g})$. The catalyst was removed by filtration and the filtrate concentrated. The resulting crude compound was washed with diethyl ether twice for removal of non-polar impurities and dried to give pure semi solid.

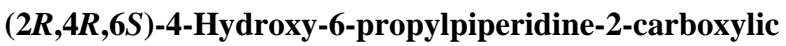
acid (Table 6 Entry 1): (21a, Starting material is 9b, Table $\mathbf{2}$, entry). Following the general procedure $\mathbf{F}$, the title compound was prepared from compound $9 \mathbf{b}(183 \mathrm{mg})$ as a white solid (88 mg, $95 \%)$. [ $\alpha]_{\mathrm{D}}{ }^{26}+40.0(c 1.0, \mathrm{MeOH})$; M.pt. 260$262{ }^{\circ} \mathrm{C} ;{ }^{1} \mathrm{H}$ NMR $\left(400 \mathrm{MHz}, \mathrm{D}_{2} \mathrm{O}\right): \delta 4.18-4.10(\mathrm{~m}, 1 \mathrm{H}), 3.88-$ $3.72(\mathrm{~m}, 2 \mathrm{H}), 2.28-2.16(\mathrm{~m}, 1 \mathrm{H}), 2.04-1.72(\mathrm{~m}, 3 \mathrm{H}), 1.71-$ $1.56(\mathrm{~m}, 2 \mathrm{H}), 1.50-1.20(\mathrm{~m}, 2 \mathrm{H}), 0.91(\mathrm{t}, J=7.2 \mathrm{~Hz}, 3 \mathrm{H})$ ppm; ${ }^{13} \mathrm{C}\left\{{ }^{1} \mathrm{H}\right\}$ NMR $\left(100 \mathrm{MHz}, \mathrm{CDCl}_{3}\right): \delta 176.6,64.7,56.0$, 53.1, 36.0, 35.6, 35.2, 21.0, $15.6 \mathrm{ppm} ; v_{\max }($ neat $) / \mathrm{cm}^{-1}: 2971$, 2489, 1596, 1393, 1096, 836, $576 \mathrm{~cm}^{-1}$; HRMS (ESI) $\mathrm{m} / \mathrm{z}$ : $[\mathrm{M}]^{+}$calcd for $\mathrm{C}_{9} \mathrm{H}_{18} \mathrm{NO}_{3}$ 188.1286; found 188.1290.

(2S,4S,6R)-4-Hydroxy-6-propylpiperidine-2-carboxylic acid (Table 6 Entry 1): (21a',Starting material is 9b', Table 2, Entry 8). Following the general procedure $\mathbf{F}$, the title compound was prepared from compound 9b' (183 mg) as a white solid (83 mg, $93 \%)$. [ $\alpha]_{\mathrm{D}}{ }^{26}=-64.0$ (c 1.0, MeOH); M.pt. 280-282 ${ }^{\circ} \mathrm{C}$; ${ }^{1} \mathrm{H}$ NMR (400 MHz, $\mathrm{D}_{2} \mathrm{O}$ ): $\delta$ 4.06-3.98 (m, $1 \mathrm{H}$ ), 
3.75-3.68 (m, $2 \mathrm{H}), 2.17-2.06$ (m, $1 \mathrm{H}), 1.90-1.63$ (m, $3 \mathrm{H})$, 1.60-1.46 (m, $2 \mathrm{H}), 1.35-1.21(\mathrm{~m}, 2 \mathrm{H}), 0.91(\mathrm{t}, J=7.2 \mathrm{~Hz}, 3$ H) ppm; ${ }^{13} \mathrm{C}\left\{{ }^{1} \mathrm{H}\right\}$ NMR $\left(100 \mathrm{MHz}, \mathrm{CDCl}_{3}\right): \delta 173.8,61.9$, $53.2,50.2,33.2,32.8,32.4,18.2,12.8 \mathrm{ppm} ; v_{\max }($ neat $) / \mathrm{cm}^{-1}$ : 2971, 1616, 1381, 1085, 940, $586 \mathrm{~cm}^{-1}$; HRMS (ESI) $\mathrm{m} / \mathrm{z}$ : $[\mathrm{M}]^{+}$calcd for $\mathrm{C}_{9} \mathrm{H}_{18} \mathrm{NO}_{3}$ 188.1286; found 188.1290.

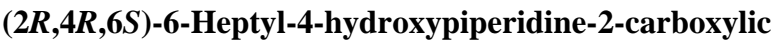
acid: (21b, Table 6 Entry 2). Following the general procedure $\mathbf{F}$, the title compound $\mathbf{2 0 b}$ was prepared from compound 9e $(211 \mathrm{mg})$ as a white solid $(110 \mathrm{mg}, 92 \%)$. $[\alpha]_{\mathrm{D}}{ }^{26}=-63.0(c$ 1.0, $\left.\mathrm{CHCl}_{3}\right)$; M.pt. $164-166{ }^{\circ} \mathrm{C} ;{ }^{1} \mathrm{H}$ NMR (400 MHz, $\left.\mathrm{D}_{2} \mathrm{O}\right): \delta$ 4.10-4.03 (m, $1 \mathrm{H}), 3.98$ (t, $J=4.4 \mathrm{~Hz}, 1 \mathrm{H}), 3.72-3.64(\mathrm{~m}, 1$ $\mathrm{H}), 2.09(\mathrm{t}, J=4.0 \mathrm{~Hz}, 3 \mathrm{H}), 1.83-1.65(\mathrm{~m}, 2 \mathrm{H}), 1.58-1.50(\mathrm{~m}$, $2 \mathrm{H}), 1.34-1.15(\mathrm{~m}, 10 \mathrm{H}), 0.72(\mathrm{t}, J=5.6 \mathrm{~Hz}, 3 \mathrm{H}) \mathrm{ppm}$; ${ }^{13} \mathrm{C}\left\{{ }^{1} \mathrm{H}\right\}$ NMR $\left(100 \mathrm{MHz}, \mathrm{CDCl}_{3}\right): \delta 172.5,61.7,52.1,49.7$, 33.5, 31.8, 31.2, 31.1, 28.2, 24.4, 22.1, $13.5 \mathrm{ppm}$; $V_{\max }($ neat $) / \mathrm{cm}^{-1}: 2971,1740,1382,1151,953,700 \mathrm{~cm}^{-1}$; HRMS (ESI) $m / z:[\mathrm{M}]^{+}$calcd for $\mathrm{C}_{13} \mathrm{H}_{26} \mathrm{NO}_{3} 244.1913$; found 244.1912 .

$(2 R, 4 R, 6 S)$-4-Acetamido-6-phenethylpiperidine-2-carboxylic acid (21c, Table 6, Entry 3). Following the general procedure $\mathbf{F}$, the title compound was prepared from compound 10a $(234 \mathrm{mg})$ as a white solid $(133 \mathrm{mg}, 91 \%)$. $[\alpha]_{\mathrm{D}}^{26}=-80.0$ (c 1.0, H2O); M.pt. $182-184{ }^{\circ} \mathrm{C} ;{ }^{1} \mathrm{H}$ NMR (400 MHz, $\left.\mathrm{D}_{2} \mathrm{O}\right): \delta$ 7.45-7.21 (m, $5 \mathrm{H}), 4.16-3.97$ (m, $2 \mathrm{H}), 3.78-3.60$ (m, $1 \mathrm{H})$, 2.88-2.67 (m, 2 H), 2.39-2.24 (m, $1 \mathrm{H}), 2.34-2.02$ (m, $4 \mathrm{H})$, 1.92 (s, $3 \mathrm{H}), 1.78-1.71$ (m, $1 \mathrm{H}) \mathrm{ppm} ;{ }^{13} \mathrm{C}\left\{{ }^{1} \mathrm{H}\right\}$ NMR (100 $\left.\mathrm{MHz}, \mathrm{CDCl}_{3}\right): \delta 173.9,140.6,129.0,128.6,126.7,51.4,41.3$, 31.1, 30.9, 30.4, 30.1, $22.0 \mathrm{ppm} ; v_{\max }$ (neat) $/ \mathrm{cm}^{-1}: 2980,1587$, 1379, 1085, $586 \mathrm{~cm}^{-1}$; HRMS (ESI) $m / z:[\mathrm{M}]^{+}$calcd for $\mathrm{C}_{16} \mathrm{H}_{23} \mathrm{~N}_{2} \mathrm{O}_{3}$ 291.1708; found 291.1711.

$(2 S, 4 S, 6 R)-4$-Acetamido-6-phenethylpiperidine-2-carboxylic acid (21c', Table 6 Entry 3). Following the general procedure $\mathbf{F}$, the title compound was prepared from compound 10a' $(234 \mathrm{mg})$ as a white solid $(121 \mathrm{mg}, 89 \%)$. $[\alpha]_{\mathrm{D}}{ }^{26}=$ +40.00 (c 1.0, MeOH); M.pt. $170-172{ }^{\circ} \mathrm{C} ;{ }^{1} \mathrm{H}$ NMR $(400 \mathrm{MHz}$, $\left.\mathrm{D}_{2} \mathrm{O}\right): \delta$ 7.32-7.03 (m, $\left.5 \mathrm{H}\right), 4.00-3.89(\mathrm{~m}, 1 \mathrm{H}), 3.78-3.69(\mathrm{~m}$, $1 \mathrm{H}), 3.60-3.52(\mathrm{~m}, 1 \mathrm{H}), 2.73-2.54(\mathrm{~m}, 2 \mathrm{H}), 2.22-2.17(\mathrm{~m}, 1$ $\mathrm{H}), 2.06-1.87$ (m, $3 \mathrm{H}), 1.81$ (s, $3 \mathrm{H}), 1.71-1.60$ (m, $2 \mathrm{H}) \mathrm{ppm}$; ${ }^{13} \mathrm{C}\left\{{ }^{1} \mathrm{H}\right\} \mathrm{NMR}\left(100 \mathrm{MHz}, \mathrm{CDCl}_{3}\right): \delta 173.6,173.3,140.6$, 128.9, 128.5, 126.6, 53.6, 51.4, 41.3, 31.1, 30.9, 30.5, 30.0, $22.0 \mathrm{ppm} ; v_{\max }($ neat $) / \mathrm{cm}^{-1}: 2981,1627,1371,749,516 \mathrm{~cm}^{-1}$; HRMS (ESI) $m / z$ : [M] $]^{+}$calcd for $\mathrm{C}_{16} \mathrm{H}_{23} \mathrm{~N}_{2} \mathrm{O}_{3}$ 291.1708; found 291.1711 .

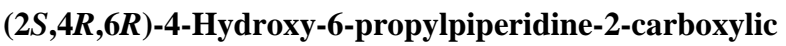
acid (21d, Table 6, Entry 4). Following the general procedure $\mathbf{G}$, the title compound $\mathbf{2 0 d}$ was prepared from compound 14a $(145 \mathrm{mg})$ as a white solid (73 mg, $76 \%)$. $[\alpha]_{\mathrm{D}}{ }^{26}=-116.0(c$ 1.0, $\left.\mathrm{CHCl}_{3}\right)$; M.pt. $246-248{ }^{\circ} \mathrm{C} ;{ }^{1} \mathrm{H}$ NMR $\left(400 \mathrm{MHz}, \mathrm{D}_{2} \mathrm{O}\right): \delta$ 4.28-3.98 (m, $2 \mathrm{H}), 3.75-3.68$ (m, $1 \mathrm{H}), 2.38-2.15$ (m, $1 \mathrm{H})$, 2.13-2.00 (m, $1 \mathrm{H}), 1.92-1.79(\mathrm{~m}, 1 \mathrm{H}), 1.70-1.46(\mathrm{~m}, 3 \mathrm{H})$, $1.35-1.28(\mathrm{~m}, 2 \mathrm{H}), 0.81$ (t, $J=6.0 \mathrm{~Hz}, 3 \mathrm{H}) \mathrm{ppm} ;{ }^{13} \mathrm{C}\left\{{ }^{1} \mathrm{H}\right\}$ NMR $\left(100 \mathrm{MHz}, \mathrm{CDCl}_{3}\right): \delta{ }^{13} \mathrm{C} 170.5,61.5,53.4,51.3,48.9$, 33.5, 31.0, 17.8, $12.7 \mathrm{ppm} ; v_{\max }$ (neat) $/ \mathrm{cm}^{-1}: 2973,1656,1451$, $1032,956,593 \mathrm{~cm}^{-1}$; HRMS (ESI) $\mathrm{m} / z$ : [M] $]^{+}$calcd for $\mathrm{C}_{9} \mathrm{H}_{18} \mathrm{NO}_{3}$ 188.1286; found 188.1290.

$(2 S, 4 R, 6 R)$-Ethyl 4-chloro-6-phenethylpiperidine-2-carboxylate: (21e, Table 6, entry 5). Following the general procedure $\mathbf{H}$, the title compound $\mathbf{2 0 e}$ was prepared from compound 12a $(185 \mathrm{mg})$ as a semi solid $(99 \mathrm{mg}, 68 \%)$. $[\alpha]_{\mathrm{D}}{ }^{26}=-$ 26.0 (c 1.0, $\mathrm{CHCl}_{3}$ ); ${ }^{1} \mathrm{H}$ NMR (400 MHz, $\left.\mathrm{D}_{2} \mathrm{O}\right): \delta$ 7.37-7.24 (m, $5 \mathrm{H}), 4.67-4.56$ (m, $1 \mathrm{H}), 4.47-4.38(\mathrm{~m}, 1 \mathrm{H}), 4.28-4.17$ (m, $1 \mathrm{H}), 3.85-3.75(\mathrm{~m}, 1 \mathrm{H}), 3.60(\mathrm{q}, J=7.2 \mathrm{~Hz}, 2 \mathrm{H}), 2.87-$ $2.48(\mathrm{~m}, 4 \mathrm{H}), 2.32-2.27(\mathrm{~m}, 1 \mathrm{H}), 2.13-1.97(\mathrm{~m}, 3 \mathrm{H}), 1.12(\mathrm{t}$, $J=6.0 \mathrm{~Hz}, 3 \mathrm{H}) \mathrm{ppm} ;{ }^{13} \mathrm{C}\left\{{ }^{1} \mathrm{H}\right\} \mathrm{NMR}\left(100 \mathrm{MHz}, \mathrm{D}_{2} \mathrm{O}\right): \delta$ 162.6, 138.6, 129.1, 128.4, 126.3, 103.7, 74.4, 71.3, 55.2, $52.2,36.1,35.4,33.9,18.6,13.8 \mathrm{ppm} ; v_{\max }($ neat $) / \mathrm{cm}^{-1}: 2956$, 1732, 1424, 1133, 983, $673 \mathrm{~cm}-1$; HRMS (ESI) $\mathrm{m} / z$ : [M] ${ }^{+}$ calcd for $\mathrm{C}_{16} \mathrm{H}_{23}{ }^{35} \mathrm{ClNO}_{2} 296.1417$; found 296.1410; calcd for $\mathrm{C}_{16} \mathrm{H}_{23}{ }^{37} \mathrm{ClNO}_{2} 298.1388$; found 298.1385.

\section{ASSOCIATED CONTENT}

Supporting Information. Preparation, screening results, characterization data, NMR spectra.

This material is available free of charge via the Internet at http://pubs.acs.org.

Accession Codes: CCDC 2014403, 1950084, 2014404 and 1950070 contain the supplementary crystallographic data for this paper. These data can be obtained free of charge via www.ccdc.cam.ac.uk/data_request/cif or by emailing data_request@ccdc.cam.ac.uk or by contacting The Cambridge Crystallographic Data Centre, 12 Union Road, Cambridge CB2 1EZ, UK; fax +44 1223336033 .

\section{AUTHOR INFORMATION}

\section{Corresponding Author}

Email: A.Dobbs@gre.ac.uk ORCID: 0000-0002-7241-7118

\section{Notes}

The authors declare no competing financial interest.

\section{ACKNOWLEDGMENT}

The authors wish to thank that European Union (Grant Number 706581 - MESO-JBIR-102; Marie Curie Individual Fellowship to RRM). The EPSRC National Mass Spectrometry Service and the University of Greenwich (Dr Iain Goodall) are gratefully acknowledged for running high resolution mass spectra. The UK National Crystallography Service ${ }^{39}$ is gratefully acknowledged for obtaining and determining all x-ray structures.

\section{REFERENCES}

1. Vitaku, E.; Smith, D. T.; Njardarson, J. T., Analysis of the Structural Diversity, Substitution Patterns, and Frequency of Nitrogen Heterocycles among US FDA Approved Pharmaceuticals. J. Med. Chem. 2014, 57, 1025710274.

2. Nebe, M. M.; Opatz, T., Synthesis of Piperidines and Dehydropiperidines: Construction of the Six-Membered Ring. Adv. Heterocyclic Chem., 2012, 122, 191-244.

3. Liu, G. Q.; Opatz, T., Recent Advances in the Synthesis of Piperidines: Functionalization of Preexisting Ring Systems. Adv. Heterocyclic Chem., 2015, 125, 107-234.

4. Dobbs, A. P.; Cuprova, L., Cascade aza-Prins reactions. Adv. Heterocyclic Chem., 2020, 130, 251-278.

5. Kandepedu, N.; Abrunhosa-Thomas, I.; Troin, Y., Stereoselective strategies for the construction of polysubstituted piperidinic compounds and their applications in natural products' synthesis. Org. Chem. Front. 2017, 4, 1655-1704.

6. Olier, C.; Kaafarani, M.; Gastaldi, S.; Bertrand, M. P., Synthesis of tetrahydropyrans and related heterocycles via 
prins cyclization; extension to aza-prins cyclization.

Tetrahedron 2010, 66, 413-445.

$7 . \quad$ Chio, F. K.; Warne, J.; Gough, D.; Penny, M.; Green, S.; Coles, S. J.; Hursthouse, M. B.; Jones, P.; Hassall, L.;

McGuire, T. M.; Dobbs, A. P., On the choice of Lewis acids for the Prins reaction; two total syntheses of (+/-)-Civet. Tetrahedron 2011, 67, 5107-5124.

8. $\quad$ Dobbs, A. P.; Parker, R. J.; Skidmore, J., Rapid access to $\mathrm{CF}_{3}$-containing heterocycles. Tetrahedron Lett. 2008, 49 (5), 827-831.

9. Zheng, K.; Liu, X. H.; Qin, S.; Xie, M. S.; Lin, L. L.; $\mathrm{Hu}, \mathrm{C}$. W.; Feng, X. M., Completely OH-Selective FeCl3Catalyzed Prins Cyclization: Highly Stereoselective Synthesis of 4-OH-Tetrahydropyrans. J. Am. Chem. Soc. 2012, 134, 17564-17573.

10. Liu, L. P.; Kaib, P. S. J.; Tap, A.; List, B., A General Catalytic Asymmetric Prins Cyclization. J. Am. Chem. Soc. 2016, 138, 10822-10825.

11. Tsui, G. C.; Liu, L. P.; List, B., The Organocatalytic Asymmetric Prins Cyclization. Angew. Chem. Int. Ed. 2015, 54, 7703-7706.

12. Lalli, C.; van de Weghe, P., Enantioselective Prins cyclization: BINOL-derived phosphoric acid and $\mathrm{CuCl}$ synergistic catalysis. Chem. Commun. 2014, 50, 7495-7498.

13. Mullen, C. A.; Gagne, M. R., Catalytic asymmetric prins cyclizations: Cation generation and trapping with (BINAP)Pt dications. Org. Lett. 2006, 8, 665-668.

14. Carballo, R. M.; Ramirez, M. A.; Rodriguez, M. L.; Martin, V. S.; Padron, J. I., Iron(III)-promoted aza-prinscyclization: Direct synthesis of six-membered azacycles. $\mathrm{Org}$. Lett. 2006, 8, 3837-3840.

15. Carballo, R. M.; Valdomir, G.; Purino, M.; Martin, V. S.; Padron, J. I., Broadening the Synthetic Scope of the Iron(III)-Catalyzed Aza-Prins Cyclization. Eur. J. Org. Chem. 2010, 2304-2313.

16. Chio, F. K. I.; Guesne, S. J. J.; Hassall, L.; McGuire, T.; Dobbs, A. P., Synthesis of Azabicycles via Cascade AzaPrins Reactions: Accessing the Indolizidine and Quinolizidine Cores. J. Org. Chem. 2015, 80, 9868-9880.

17. Dobbs, A. P.; Guesne, S. J. J.; Parker, R. J.; Skidmore, J.; Stephenson, R. A.; Hursthouse, M. B., A detailed investigation of the aza-Prins reaction. Org. Biomol. Chem. 2010, 8, 1064-1080.

18. Miranda, P. O.; Carballo, R. M.; Martin, V. S.; Padron, J. I., A New Catalytic Prins Cyclization Leading to Oxa- and Azacycles. Org. Lett. 2009, 11, 357-360.

19. Murty, M. S. R.; Ram, K. R.; Yadav, J. S., BiCl(3) promoted aza-Prins type cyclization: a rapid and efficient synthesis of 2,4-disubstituted piperidines. Tetrahedron Lett. 2008, 49, 1141-1145.

20. Reddy, B. V. S.; Nair, P. N.; Antony, A.; Lalli, C.; Gree, R., The Aza-Prins Reaction in the Synthesis of Natural Products and Analogues. Eur. J. Org. Chem. 2017, 1805-1819.

21. Reddy, B. V. S.; Ramesh, K.; Ganesh, A. V.; Kumar, G.; Yadav, J. S.; Gree, R., Triflic acid-promoted aza-PrinsRitter reaction sequence: a novel synthesis of 4amidopiperidine derivatives. Tetrahedron Lett. 2011, 52, 495498.
22. Diez-Poza, C.; Barbero, A., Synthesis of O- and NHeterocycles by Silyl-Prins Cyclization of Allylsilanes. Eur. J. Org. Chem. 2017, 4651-4665.

23. Dobbs, A. P.; Guesne, S. J. J.; Hursthouse, M. B.; Coles, S. J., The aza-silyl-prins reaction: A novel method for the synthesis of Trans-2,6-tetrahydropyridines. Synlett 2003, 1740-1742.

24. Dobbs, A. P.; Martinovic, S. A., The silyl-Prins reaction: a novel method for the synthesis of dihydropyrans. Tetrahedron Lett. 2002, 43, 7055-7057.

25. Dobbs, A. P.; Guesne, S. J. J.; Martinovic, S.; Coles, S. J.; Hursthouse, M. B., A versatile indium trichloride mediated Prins-type reaction to unsaturated heterocycles. $J$. Org. Chem. 2003, 68, 7880-7883.

26. Royer, J.; Bonin, M.; Micouin, L., Chiral heterocycles by iminium ion cyclization. Chem. Rev. 2004, 104, 2311-2352.

27. Mittapalli, R. R.; Guesne, S. J. J.; Parker, R. J.; Klooster, W. T.; Coles, S. J.; Skidmore, J.; Dobbs, A. P., The Asymmetric Aza-silyl-Prins Reaction: Synthesis of Enantiopure Piperidines. Org. Lett. 2019, 21, 350-355.

28. Bai, J. F.; Yasumoto, K.; Kano, T.; Maruoka, K., Synthesis of 1-Aminoindenes through Aza-Prins-Type Cyclization. Chem. Eur. J. 2018, 24, 10320-10323.

29. Durel, V.; Lalli, C.; Roisnel, T.; van de Weghe, P., Synergistic Effect of the TiCl4/p-TsOH Promoter System on the Aza-Prins Cyclization. J. Org. Chem. 2016, 81, 849-859.

30. Dobbs, A. P.; Cuprova, L., Cascade aza-Prins reactions. Adv. Heterocyclic. Chem. 2020, 130, 251-278.

31. Dobbs, A. P.; Guesne, S. J. J., Rapid access to trans2,6-disubstituted piperidines: Expedient total syntheses of (-)solenopsin A and (+)-epi-dihydropinidine. Synlett 2005, 21012103.

32. Takatori, K.; Nishihara, M.; Kajiwara, M., An asymmetric synthesis of L- 2-C-13 aspartic acid from sodium 2-C-13 acetate. J. Labelled Cpds \& Radiopharm. 1999, 42, 701-708.

33. Williams, R. M.; Sinclair, P. J.; Zhai, D.; Chen, D., Practical asymmetric synthesis of $\alpha$-amino acids through carbon-carbon bond constructions on electrophilic glycine templates. J. Am. Chem. Soc. 1988, 110, 1547-1557.

34. Williams, R. M.; Sinclair, P. J.; D.E., D.; Zhai, D.; Chen, D., Asymmetric synthesis of $N$-tert-butoxycarbonyl $\alpha$ amino acids. Synthesis of $(5 S, 6 R)$-4-tert-butoxycarbonyl-5,6diphenylmorpholin-2-one. Org. Synth. 2003, 80, 18-30.

35. Deposited with the Cambridge Crystallographic Data Centre CCDC 2014403.

36. Indukuri, K.; Unnava, R.; Deka, M. J.; Saikia, A. K., Stereoselective Synthesis of Amido and Phenyl Azabicyclic Derivatives via a Tandem Aza Prins-Ritter/Friedel-Crafts Type Reaction of Endocyclic $N$-Acyliminium Ions. J. Org. Chem. 2013, 78, 10629-10641.

37. Deposited with the Cambridge Crystallographic Data Centre CCDC 1950084.

38. Deposited with the Cambridge Crystallographic Data Centre. 17c: CCDC 2014404 and 19b: CCDC 1950070.

39. Coles, S. J.; Gale, P. A., Changing and challenging times for service crystallography. Chem. Sci. 2012, 3, 683689. 



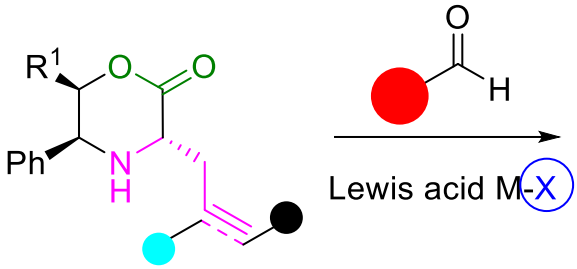

homoallylic or

homopropargylic amine

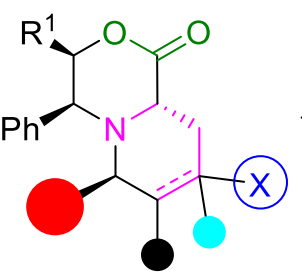
$\mathrm{H}_{2}, \mathrm{Pd} / \mathrm{C}$
EtOH, r.t., $12 \mathrm{hr}$

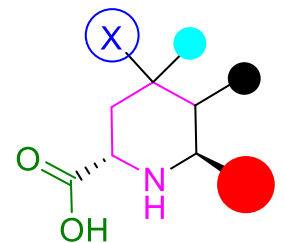

piperidines and pipecolic acids single enantiomer and diastereomer 